\title{
Buildings and their applications in geometry and topology
}

\author{
Lizhen Ji \\ Department of Mathematics \\ University of Michigan \\ Ann Arbor, MI 48109
}

February 2, 2006

\begin{abstract}
Buildings were first introduced by J.Tits in 1950s to give systematic geometric interpretations of exceptional Lie groups and have been generalized in various ways: Euclidean buildings (Bruhat-Tits buildings), topological buildings, $\mathbb{R}$-buildings, in particular $\mathbb{R}$-trees. They are useful for many different applications in various subjects: algebraic groups, finite groups, finite geometry, representation theory over local fields, algebraic geometry, Arakelov intersection for arithmetic varieties, algebraic $K$-theories, combinatorial group theory, global geometry and algebraic topology, in particular cohomology groups, of arithmetic groups and S-arithmetic groups, rigidity of cofinite subgroups of semisimple Lie groups and nonpositively curved manifolds, classification of isoparametric submanifolds in $\mathbb{R}^{n}$ of high codimension, existence of hyperbolic structures on three dimensional manifolds in Thurston's geometrization program. In this paper, we survey several applications of buildings in differential geometry and geometric topology.
\end{abstract} There are four underlying themes in these applications:

1. Buildings often describe the geometry at infinity of symmetric spaces and locally symmetric spaces and also appear as limiting objects under degeneration or scaling of metrics.

2. Euclidean buildings are analogues of symmetric spaces for semisimple groups defined over local fields and their discrete subgroups.

3. Buildings of higher rank are rigid and hence objects which contain or induce higher rank buildings tend to be rigid.

4. Additional structures on buildings, for example, topological buildings, are important in applications for infinite groups.

\section{Contents}

1 Introduction and history of buildings $r$

$\begin{array}{lll}2 & \text { Spherical Tits buildings } & 7\end{array}$

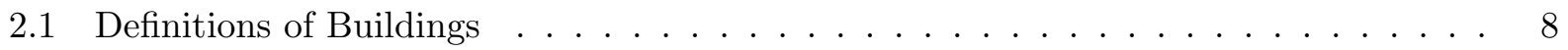

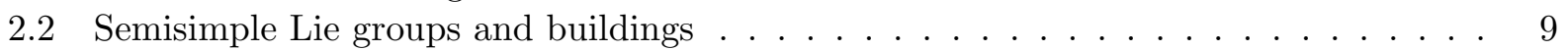

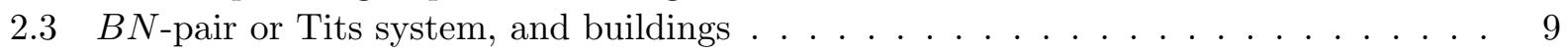

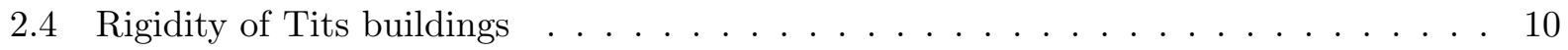

2.5 Geodesic compactification of symmetric spaces . . . . . . . . . . . . . . 11

2.6 Buildings and compactifications of symmetric spaces . . . . . . . . . . . . 12

2.7 Topological spherical Tits buildings . . . . . . . . . . . . . . . . 15

2.8 Mostow strong rigidity . . . . . . . . . . . . . . . . 16 
2.9 Rank rigidity of manifolds of nonpositive curvature . . . . . . . . . . . . 20

2.10 Classification of isoparametric submanifolds . . . . . . . . . . . . . . 22

2.11 Spherical buildings and compactifications of locally symmetric spaces . . . . . . . 25

2.12 Geodesic compactification, Gromov compactification and large scale geometry . . . . 28

3 Euclidean buildings $\quad 29$

3.1 Definitions and basic properties . . . . . . . . . . . . . 30

3.2 Semisimple $p$-adic groups and Euclidean Buildings $\ldots \ldots \ldots \ldots$. . . . . . . 31

$3.3 p$-adic curvature and vanishing of cohomology . . . . . . . . . . . . . . . 34

3.4 Super-rigidity and harmonic maps into Euclidean buildings . . . . . . . . . . . . 35

3.5 Compactification of Euclidean buildings by spherical buildings . . . . . . . . . . 40

3.6 Applications to S-arithmetic groups . . . . . . . . . . . . . . . . . . . 42

$4 \quad \mathbb{R}$-trees and $\mathbb{R}$-buildings $\quad \mathbf{4 5}$

4.1 Definition of $\mathbb{R}$-trees and basic properties . . . . . . . . . . . . . . . 45

4.2 Applications of $\mathbb{R}$-trees in topology . . . . . . . . . . . . . . . . 47

$4.3 \mathbb{R}$-Euclidean buildings . . . . . . . . . . . . . . . . . 51

4.4 Quasi-isometries and tangent spaces at infinity of symmetric spaces . . . . . . . . 53

5 Other applications of buildings $\quad 54$

5.1 Applications in algebraic geometry . . . . . . . . . . . . . . . . . 54

5.2 Applications in representation theory . . . . . . . . . . . . . . 56

5.3 Random walks and the Martin boundary . . . . . . . . . . . . . . . . 56

5.4 Finite groups, finite geometry and algebraic $K$-groups $\ldots \ldots \ldots$. . . . . . . 56

5.5 Hyperbolic buildings and combinatorial group theory . . . . . . . . . . . . 57

5.6 Algebraic combinatorics . . . . . . . . . . . . . . . . . 57

5.7 Expanders and Ramanujan graphs . . . . . . . . . . . . . . . . . . 57

\section{Introduction and history of buildings}

In 1872, at the tender age of 23, Felix Klein assumed his professorship and delivered his inaugural address at the University of Erlangen with a title Comparative review of recent results in geometry [Kle]. In the talk, he proposed a program to understand geometry from the point of view of groups and hence to uniformize and classify different geometries. This became known as the famous Erlangen program, which was summarized in one sentence by Klein: Geometry is the science which studies the properties of figures preserved under the transformation of a certain group of transformations, or, as one also says, the science which studies the invariants of a group of transformations.

The Erlangen program has had profound impact in the development of mathematics. In fact, the philosophy of finding and understanding invariants of automorphism groups of any system or structure can be found in many branches of mathematics, for example, algebraic topology. This point of view has also been very useful in physics and other sciences.

There are two implications in the Erlangen program:

1. Given a geometry, for example, the Euclidean geometry, the hyperbolic geometry or the projective geometry, find the transformation group which preserves properties of the figures in this geometry. 
2. Conversely, given a group, find a geometry such that the group acts on it and preserves its geometric properties.

In general, we expect the group to be sufficiently large, for example, the action is transitive and the space becomes a homogeneous space of the group. In the Erlangen program, it is not only the total transformation group which is important. Subgroups, such as stabilizers of points or figures are integral parts of the description of geometry in terms of groups. For example, different homogeneous spaces of a Lie group are determined by the stabilizers of points in the spaces; in the projective geometry, the incidence relation between points, lines and subspaces in projective spaces can be described in terms of their stabilizers in the projective linear group.

Besides the stabilizer subgroups of points and figures, other subgroups, for example, which preserve some structures on the space, are also important. In fact, Klein obtained the Euclidean geometry and hyperbolic geometry as specializations of the projective geometry by using suitable subgroups of the projective linear group (see [Kli] for more detail).

It is known that the family of simple (complex) Lie groups (or algebras) consists of

1. four classical infinite series: $\left(A_{n}\right), S L(n+1, \mathbb{C}) ;\left(B_{n}\right), S O(2 n+1, \mathbb{C}) ;\left(C_{n}\right), S p(n, \mathbb{C}) ;\left(D_{n}\right)$, $S O(2 n, \mathbb{C})$,

2. and five exceptional ones: $E_{6}, E_{7}, E_{8}, F_{4}$ and $G_{2}$.

The classical ones correspond to classical geometry in the sense that their compact real forms $S U(n), S O(n)$ and $S p(n)$ correspond to the identity component of the isometry groups of the projective spaces $\mathbb{C} P^{n-1}, \mathbb{R} P^{n-1}$ and $\mathbb{H} P^{n-1}$, where $\mathbb{H}$ is the algebra of quaternions.

The exceptional groups correspond to the automorphism groups of the projective planes over the algebras $\mathbb{O}, \mathbb{O} \otimes \mathbb{C}, \mathbb{O} \otimes \mathbb{H}$ and $\mathbb{O} \otimes \mathbb{O}$, where $\mathbb{O}$ is the algebra of octonions (or Cayley numbers). But due to the non-associativity of $\mathbb{O}$, these spaces do not satisfy the usual axioms for a projective plane, and some other geometric models are needed for the exceptional Lie groups (see [Bae] for a nice summary of the geometry of these spaces and relations to the exceptional groups).

To carry out the Erlangen program for the exceptional groups and also to realize exceptional groups over other fields, for example over finite fields in order to get finite groups of exceptional Lie types, Tits introduced spherical buildings. It should probably be emphasized that in the theory of Tits buildings (or Tits geometry), it is the incidence geometry but not the metric geometry which is emphasized. In the incidence geometry, a crucial concept is the incidence relation which is the inclusion relation between distinguished subspaces [Bue]. (Note that the incidence relation between points, lines and other linear subspaces is a crucial part of the projective geometry over $\mathbb{R}$ and $\mathbb{C}$ ).

Spherical Tits buildings were first introduced by Tits in a series of papers from 1950s on (see [Ti4] [Ti5] [Ti6]), which resulted in the fundamental book [Ti2]. They are simplicial complexes which contain a collection of distinguished sub-complexes called apartments, which are finite triangulations of spheres (see $\S 2$ below for more details). For any semisimple linear algebraic group $\mathbf{G}$ defined over any field $k$, there is a spherical building $\Delta(\mathbf{G})$ whose simplexes correspond to (proper) parabolic subgroups of $\mathbf{G}$ defined over $k$. Let $r$ be the $k$-rank of $\mathbf{G}$, i.e., the maximal dimension of $k$-split tori contained in $\mathbf{G}$. Then $\Delta(\mathbf{G})$ is a simplicial complex of dimension $r-1$ and is called a building of rank $r$. If the field $k$ is finite, the building is a finite simplicial complex. Otherwise, it is infinite in general. Clearly, $\mathbf{G}(k)$ acts on the set of parabolic subgroups defined over $k$ and hence acts on $\Delta(\mathbf{G})$ by simplicial automorphisms. This gives the first implication in the Erlangen program: Constructing geometries from groups.

On the other hand, given a (spherical) building $\Delta$, its automorphism group provides interesting examples of groups. Once buildings can be constructed independently from groups, it gives a 
geometric method to construct groups (see for example [RT] [Ro1]). An important result of Tits [Ti2] shows that a thick building of rank at least 3 arises from a semisimple algebraic group and in this case the group is completely determined by the building. This shows that the group is described by the geometry and gives the second implication of the Erlangen program: Constructing groups from geometries.

An effective way to construct buildings for a group $G$ is to use a BN-pair, a pair of subgroups $B$ and $N$ of $G$ which satisfy certain conditions. Such pairs are often called a Tits system.

In [IM], Iwahori and Matsumoto introduced a new type of BN-pairs in Chevalley groups defined over a non-archimedean local field, i.e., a locally compact field with a discrete valuation. This leads to a new type of building in which an apartment is a triangulation of a Euclidean space. These buildings are called Euclidean buildings. Later, Bruhat and Tits [BT1] constructed a Euclidean building $\Delta^{B T}(\mathbf{G})$ for a linear (simply connected) semisimple (or reductive) algebraic group $\mathbf{G}$ defined over a non-archimedean local field $k$. Due to this result, Euclidean buildings are often called Bruhat-Tits buildings. The dimension of the Bruhat-Tits building $\Delta^{B T}(\mathbf{G})$ is equal to $r$, the $k$-rank of the algebraic group $\mathbf{G}$. When the rank $r$ is equal to 1 , the building $\Delta^{B T}(\mathbf{G})$ becomes a tree. The Bruhat-Tits building $\Delta^{B T}(\mathbf{G})$ and the spherical Tits building $\Delta(\mathbf{G})$ are closely related. In fact, the latter appears at the infinity of the former.

The primary properties of buildings are of combinatorial nature, for example, the incidence relations between simplexes. Since apartments can be endowed with metrics which are compatible on their intersection, these metrics can be glued together into a canonical metric, called the Tits metric, on the building. With respect to this topology, Euclidean buildings and infinite spherical Tits buildings are noncompact. It turns out that locally compact Euclidean buildings can be compactified by adding at infinity suitable spherical Tits buildings which are given a topology different from the one induced by its canonical Tits metric $[\mathrm{BoS}]$. When $\mathbf{G}$ is a linear semisimple algebraic group defined over a non-archimedean local field $k$, then the Bruhat-Tits building $\Delta^{B T}(\mathbf{G})$ is locally compact, and the spherical Tits building which compactifies $\Delta^{B T}(\mathbf{G})$ is exactly the building $\Delta(\mathbf{G})$ with a suitable topology. (As will be seen below, the two buildings $\Delta(\mathbf{G})$ and $\Delta^{B T}(\mathbf{G})$ arise from two different choices of $B N$-pairs in $\mathbf{G}(k)$, or rather two different choices of $B$ but the same $N$ ).

In fact, with respect to the topology induced from the Tits metric, the spherical building is not compact unless it is a finite simplicial complex, and hence it is important to give the spherical building a different topology. Besides this natural application to relate two kinds of buildings, this compactification is crucial for the study of cohomology groups of S-arithmetic subgroups in [BoS1]. When the Euclidean building is a tree, this compactification is the well-known compactification of trees by the space of ends.

Spherical buildings with this new topology are called topological buildings by Burns and Spatzier in [BuS2] (actually, the topological buildings were defined slightly differently there) and were used by them to prove rank rigidity of complete manifolds of nonpositive curvature and finite volume in [BuS1]. They were also used by Thorbergsson [Tho2] to classify compact isoparametric submanifolds in $\mathbb{R}^{n}$ of codimension at least three. One point we want to emphasize in this article is that this additional structure of a topology on the spherical Tits buildings is natural and important. In fact, since Tits buildings are combinatorial objects, their automorphism groups do not have natural topologies and are basically groups with the discrete topology. The additional topology on the buildings allows us to conclude that the automorphism group (or its identity component) is a Lie group, which leads to the desired rigidity results or classifications in differential geometry.

Perhaps it is worthwhile to point out that the first major application of the spherical Tits buildings to geometry is in the proof of the Mostow strong rigidity [Mos]. Though the topological 
spherical Tits buildings were not introduced or used in [Mos], the basic idea of topological buildings played an important role there. In fact, the combination of an isomorphism of the two spherical Tits buildings associated with the two Lie groups in question and the homeomorphism on their maximal Furstenberg boundaries is needed to prove the isomorphism between the two Lie groups, i.e., the desired Mostow strong rigidity. The point is that the maximal Furstenberg boundaries can naturally be identified with the spaces of the simplexes of maximal dimension (or chambers) of the spherical Tits buildings, and the homeomorphism between them is part of an isomorphism between two topological Tits buildings.

In Thurston's geometrization program of three dimensional manifolds [Thu1] [Thu2] [Thu3], compactness of the space of hyperbolic structures of certain manifolds is crucial. Morgan and Shalen [MS1] [MS2] [Mor2] showed that if noncompact, such spaces of hyperbolic structures can be compactified by $\mathbb{R}$-trees admitting certain action of discrete groups. By analyzing such actions, they could reprove the compactness of the spaces of hyperbolic structures in [Thu1] (see also [Kap]). $\mathbb{R}$-trees also played an important role in the proof by Otal [Ot2] of a result of Thurston on existence of the hyperbolic metric on certain 3-manifolds fibered over the circle $S^{1}$. The combination of these two results proves the Thurston hyperbolization theorem [Kap] [Ot1] [Ot2]. $\mathbb{R}$-trees have also been used in other related problems in geometry, topology and combinatorial group theory. In fact, $\mathbb{R}$-trees were used to understand the automorphism groups of and the topology of the boundaries of Gromov-hyperbolic groups; see [Bes1] [Pau1] [BF] for surveys of these applications together with relations to measured foliations. See also [KlS] [AS] [Wol1] [DDW] for other applications.

The $\mathbb{R}$-trees also occur naturally in algebraic group theories. In fact, Bruhat and Tits [BT1, $\S 7.4]$ defined $\mathbb{R}$-Euclidean buildings which are not simplicial complexes for linear semisimple simply connected algebraic groups over fields with non-discrete valuations. When the rank of the algebraic groups is equal to 1 , the $\mathbb{R}$-Euclidean buildings are $\mathbb{R}$-trees.

The $\mathbb{R}$-trees in [MS1] [MS2] can be interpreted as the tangent cone at infinity of the real hyperbolic space. Naturally, the tangent cone at infinity of higher rank symmetric spaces of noncompact type is expected to lead to $\mathbb{R}$-Euclidean buildings. In fact, they were introduced and used by Kleiner and Leeb in [KL2] to prove the rigidity of irreducible symmetric spaces of rank at least two under quasi-isometries, a conjecture of Margulis. For completeness, we mention that the tangent cone at infinity of other rank- 1 symmetric spaces of non-compact type is also $\mathbb{R}$-trees, and the tangent cone at infinity of a (usual) Euclidean building is also an $\mathbb{R}$-Euclidean building, and the results in [KL2] also hold for irreducible Euclidean buildings of rank at least two.

Symmetric spaces of noncompact type have played an important role in understanding structures of semisimple Lie groups and its subgroups, for example, the conjugacy of maximal compact subgroups, and the cohomology groups and other problems of discrete subgroups. For linear semisimple algebraic groups over non-archimedean local fields, the analogues of the symmetric spaces are the Bruhat-Tits buildings. In fact, the buildings have been used to understand compact open subgroups and cohomology groups of S-arithmetic subgroups.

An important application of the Bruhat-Tits buildings in geometry is the proof by Gromov and Schoen [GS] of the non-archimedean super-rigidity of co-finite discrete subgroups of $S p(1, n)$ and $F_{4(-20)}$, the isometry groups of the quaternionic hyperbolic spaces and the Cayley hyperbolic plane. In fact, they developed a theory of harmonic maps from Riemannian manifolds into the Euclidean buildings for this purpose. This result was not covered by the famous Margulis super-rigidity theorem [Mar].

All the buildings mentioned above appear naturally in geometry and topology. There is a further class of buildings called Moufang buildings. Briefly, they are buildings with large (root) automorphisms. Spherical buildings of rank at least 3 and Bruhat-Tits buildings over power series fields 
are Moufang buildings. But there are other Moufang buildings, for example, those associated with infinite dimensional Kac-Moody algebras in [MT]. In fact, they showed that any crystallographic Coxeter group can be realized as the Weyl group of suitable $B N$-pairs of the automorphism group of some (infinite dimensional) Kac-Moody algebras. Many crystallographic Coxeter groups arise from reflections in the real hyperbolic spaces (see [Vi] [VS] [Ni1] [Ni2] [JS]), in which case the apartment in the building has negative curvature and the building is called a hyperbolic building (see [Re3] and its references). We remark (see below) that apartments in spherical buildings have positive curvature, and apartments in Euclidean buildings have zero curvature. Some rigidity results similar to the rigidity of quasi-isometries in [KL2] have been developed for certain hyperbolic buildings in [BP2]. See [BP2] for a survey of related results. On the other hand, hyperbolic buildings have played an increasing important role in combinatorial group theory [CG1] [CG2] [Lu] [Re1] [Re2] [Re4] [DJ] [Ja] [Laf1] [Laf2].

For the organization of paper and the topics discussed, see the table of contents at the beginning of this paper. On the other hand, in the rest of the introduction, we outline the main divisions of this paper and the rationales for them.

In $\S 2$, we discuss the spherical Tits buildings, and the enhanced topological buildings.

The Mostow rigidity and the rank rigidity of manifolds of nonpositive curvature deal with locally symmetric spaces. They use the spherical Tits buildings of semisimple Lie groups (or symmetric spaces) and are put in the first part of $\S 2$. Though the Margulis super-rigidity is a natural continuation of the Mostow strong rigidity, it involves $p$-adic Lie groups and hence BruhatTits buildings. Due to this reason, it is put in $\S 3$.

The second part of $\S 2$ deals with applications of the spherical Tits buildings to the large scale geometry and compactifications of locally symmetric spaces defined by arithmetic subgroups.

In $\S 3$, we discuss Euclidean buildings and applications to the Margulis super-rigidity and the integral Novikov conjectures of S-arithmetic subgroups.

After discussing the usual spherical and Euclidean buildings in $\S 2$ and $\S 3$, we discuss $\mathbb{R}$-Euclidean buildings, which are non-discrete analogues of usual Euclidean buildings in $\S 4$. Since $\mathbb{R}$-trees are very important special cases of $\mathbb{R}$-Euclidean buildings, we discuss them and their applications first. Then we recall the general $\mathbb{R}$-Euclidean buildings and a method in [BT1, §7.4] to construct them from algebraic groups over fields with non-discrete valuations. This method can also be used to construct the usual (simplicial) buildings.

Though this paper mainly discusses applications of buildings in geometry and topology, we indicate briefly other applications in algebraic geometry, representation theories of $p$-adic groups, and potential theory (random walks and Martin compactifications) on buildings, algebraic combinatorics and finite geometry etc together with (hopefully) adequate references.

Before concluding the introduction, we mention that besides the original papers and expository writings of Tits (for example [Ti1]) and Bruhat, there are also excellent books [Br1] [Br2] [Ro3] [Garr] [TW], survey articles [Ro1] [Ro2] [Sca] [RS] and books consisting of expository articles on buildings and incidence geometries [Bue] [KLPS] [Ros]. A natural problem is to justify this survey article. In fact, many applications of buildings in geometry and topology occurred after the books [Br1] [Ro3] were written. The Borel-Serre compactification of locally symmetric spaces and its applications to cohomology groups were discussed in [Br1] [Ro2] [RS], and the applications of the topological spherical Tits buildings to the rank rigidity of nonpositively curved manifolds and the isoparametric manifolds were briefly mentioned in [RS]. We discuss mostly about applications not covered in these articles and books, and hence the overlap with them is minimal. More importantly, we believe that the enhanced topological buildings are more natural and important than the usual combinatorial buildings for applications in differential geometry and topology; and it is instructive 
and helpful to study and compare three types of buildings simultaneously: the (usually locally finite) combinatorial buildings, topological buildings, and (non-locally finite) $\mathbb{R}$-buildings and the particularly important special class of $\mathbb{R}$-trees.

\section{Acknowledgments:}

The initial email correspondences with K.Brown about applications of buildings to geometry motivated me to write this survey. I would like to thank him for his encouragement and support for this project and for his very careful reading and comments on the preliminary versions of this paper. I would also like to thank G.Prasad, R.Spatzier, I.Dolgachev for helpful conversations, comments and for references on buildings, rigidity of locally symmetric spaces and algebraic geometry, D.Canary for detailed comments, suggestions and references about more recent applications of $\mathbb{R}$ trees in hyperbolic geometry, and J.Souto for helpful conversations on ultralimits and $\mathbb{R}$-trees. I would also like to thank S.T.Yau for precise and very helpful comments on applications of harmonic maps to rigidity problems of complex manifolds and locally symmetric spaces, R.Lazarsfeld for the reference [FJ], S.K.Yeung for explanations of and the references [Ye1] [Ye2] [Klig1] on recent results about the rigidity of lattices acting on the complex hyperbolic plane, S.Fomin for the references in algebraic combinatorics, and C.L.Terng for very helpful comments and suggestions in Remark 2.10.6 and the references [Tho1] [FKM], V.Reiner for the reference [McS] [Tha1] [Tha2], B.Conrad for the references [Ber2] $[\mathrm{BoC}]$ and clarifying notions of p-adic analytic spaces, E.Loojenga for helpful conversations and the reference [Del], Winnie W.C.Li for references on applications of trees and buildings to Ramanujan graphs. This work is partially supported by NSF grant DMS 0405884.

\section{Spherical Tits buildings}

In this section, we recall the definition of spherical Tits building and the related topological Tits buildings, applications to rigidity problems in differential geometry, and global geometry and compactifications of symmetric and locally symmetric spaces.

More specifically, we construct the spherical Tits building of a semisimple algebraic group over any field using parabolic subgroups, and the general method using $B N$-pairs or Tits systems. For applications to rigidity problems, we recall two main results of Tits in [Ti2]. For real semisimple Lie groups, we realize their spherical buildings in terms of the sphere at infinity of the corresponding symmetric space of noncompact type. This naturally leads to the concept of topological Tits buildings. Then we recall the maximal Satake compactification of symmetric spaces in order to introduce the maximal Furstenberg boundary, which is used crucially in the proof of the Mostow strong rigidity, and explain relations between the boundaries of compactifications of symmetric spaces and the Tits buildings in [GJT] [BJ1].

Then we outline the proof of the Mostow strong rigidity in the higher rank case, which will motivate the Margulis super-rigidity in $\S 3$ and the Margulis conjecture on quasi-isometries between symmetric spaces and quasi-flats in $\S 4$.

To illustrate the power of topological Tits buildings, we discuss their applications to the problem of rank rigidity of manifolds of nonpositive curvature and finite volume, and the classification of compact isoparametric submanifolds of codimension at least 3 in Euclidean spaces. These are major applications of the (topological) Tits buildings of real Lie groups.

Then we discuss the spherical Tits buildings of semisimple linear algebraic groups defined over $\mathbb{Q}$. Quotients of the Tits buildings by arithmetic subgroups occur naturally as the boundary of a compactification of the arithmetic locally symmetric spaces, called the Tits compactification, which is homeomorphic to the Gromov compactification and the geodesic compactification of locally 
symmetric spaces, and is also related to the tangent cone at infinity of the locally symmetric spaces and the Siegel conjecture on comparison of metrics on Siegel sets.

\subsection{Definitions of Buildings}

In this paper, all simplicial complexes are assumed to be finite dimensional.

Definition 2.1.1 A simplex in a simplicial complex is called a chamber if it is maximal, i.e., not contained in another simplex as a face. Two simplexes are called adjacent if they have a common face of codimension 1 (i.e., a facet) and hence of the same dimension. A simplicial complex is called a chamber complex if all chambers have the same dimension, and any two chambers $C, C^{\prime}$ are connected by a gallery, i.e., a sequence of chambers $C_{1}, \cdots, C_{n}$ such that $C_{1}=C, C_{n}=C^{\prime}$, and $C_{i}, C_{i+1}$ are adjacent for $i=1, \cdots, n-1$.

For example, a tree is a 1-dimensional chamber complex. But two 2-simplexes connected by a 1-simplex in the shape of dumbbell is not.

Definition 2.1.2 A finite group $W$ acting isometrically on a Euclidean space $V$ is called a finite reflection group if it is generated by reflections with respect to hyperplanes.

We emphasize that the elements in $W$ act as linear transformations. Assume that $W$ acts essentially on $V$ in the sense that there is no nontrivial linear subspace which is fixed by $W$ (see [Br1, Chap I. $\S 1$, Chap II. 4, Chap III] for details of definitions and discussions below). Then $W$ is a finite Coxeter group, and any abstract finite Coxeter group can be realized this way.

Given any finite Coxeter group $W$, there is a Coxeter complex, a finite simplicial complex which can be constructed as follows. Every reflection $\alpha \in W$ fixes a hyperplane $\mathcal{H}_{\alpha}$. The collection of such hyperplanes $\mathcal{H}_{\alpha}$ is invariant under $W$. Connected components of their complements in $V$ are called chambers, which are simplicial cones. The chambers and their faces together give a partition of $V$ into simplicial cones. Let $S$ be the unit sphere in $V$. Then the intersection of $S$ with these simplicial cones gives a finite simplicial complex, called the Coxeter complex of $W$, whose underlying topological space is $S$, i.e., a finite triangulation of the unit sphere. It can be seen that a Coxeter complex is a chamber complex.

Definition 2.1.3 A simplicial complex $\Delta$ is called a spherical Tits building if it contains a family of subsets called apartments and satisfies the following conditions:

1. Every apartment is a finite Coxeter complex.

2. Any two simplexes are contained in some apartment.

3. Given two apartments $\Sigma$ and $\Sigma^{\prime}$ and simplexes $\sigma, \sigma^{\prime} \in \Sigma \cap \Sigma^{\prime}$, there exists an isomorphism of $\Sigma$ onto $\Sigma^{\prime}$ which keeps $\sigma, \sigma^{\prime}$ pointwise fixed.

The condition (3) implies that there is a common Coxeter group whose complex gives the apartments. Since the apartments are triangulations of the unit sphere, the building is called spherical. It also implies that $\Delta$ is a chamber complex.

A spherical Tits building $\Delta$ is called thick if every simplex of codimension one is contained in at least three chambers. In the following, all buildings are assumed to be thick unless indicated otherwise. The rank of the building $\Delta$ is defined to be $\operatorname{dim} \Delta+1$. 


\subsection{Semisimple Lie groups and buildings}

An important example of spherical Tits buildings is constructed from a semisimple algebraic group $\mathbf{G}$ over a field $k$ as follows.

Recall that a subgroup $\mathbf{P}$ of $\mathbf{G}$ defined over $k$ is called a $k$-parabolic subgroup of $\mathbf{G}$ if $\mathbf{G} / \mathbf{P}$ is a projective variety. For example, when $\mathbf{G}=S L(n)$, the subgroup $\mathbf{B}$ consisting of upper triangular matrices is a $k$-parabolic subgroup. In fact, any $k$-subgroup $\mathbf{P}$ containing $\mathbf{B}$ is a $k$-parabolic subgroup, and any $k$-parabolic subgroup is conjugate to one which contains $\mathbf{B}$.

Let $\Delta=\Delta(\mathbf{G})$ be the simplicial complex whose simplexes correspond to proper $k$-parabolic subgroups as follows:

1. The vertexes of $\Delta$ correspond to maximal (proper) $k$-parabolic subgroups of $\mathbf{G}$.

2. Vertexes $\mathbf{Q}_{1}, \cdots, \mathbf{Q}_{m}$ form the vertexes of a simplex $\sigma$ if and only if the intersection $\mathbf{Q}_{1} \cap$ $\cdots \cap \mathbf{Q}_{m}$ is a $k$-parabolic subgroup, which corresponds to the simplex $\sigma$.

The apartments in the building $\Delta(\mathbf{G})$ are constructed as follows. For any maximal $k$-split torus $\mathbf{T}$ of $\mathbf{G}$, there are only finitely many $k$-parabolic subgroups containing $\mathbf{T}$, and their corresponding simplexes in $\Delta$ form a Coxeter complex whose Coxeter group is given by the Weyl group $W$ of $\mathbf{T}$, where $W=N(\mathbf{T}) / \mathbf{T}$, where $N(\mathbf{T})$ is the normalizer of $\mathbf{T}$ in $\mathbf{G}$ (see [Ti2]). In this building, chambers correspond to minimal parabolic subgroups. Clearly, the group $\mathbf{G}(k)$ of $k$-points acts on the set of $k$-parabolic subgroups by conjugation and hence acts on the building $\Delta(\mathbf{G})$ by simplicial automorphisms. The rank of the building $\Delta(\mathbf{G})$ is equal to the $k$-rank $r$ of $\mathbf{G}$.

Remark 2.2.1 In this section, we are only concerned with two cases: either $k=\mathbb{R}$ or $k=\mathbb{Q}$. In the former case, instead of the algebraic group $\mathbf{G}$ defined over $\mathbb{R}$, we could consider only the real locus $G=\mathbf{G}(\mathbb{R})$, a Lie group with finitely many connected components, and the parabolic subgroups of the Lie group $G$. In the second case, we need the structure of the algebraic group $\mathbf{G}$ defined over $\mathbb{Q}$ in order to study the boundary components for locally symmetric spaces defined by arithmetic subgroups.

To show that the simplicial complex $\Delta(\mathbf{G})$ defined above satisfies the conditions in Definition 2.1 , we need the Bruhat decomposition to understand the structure of $k$-parabolic subgroups. It turns out that they are best described in terms of $B N$-pairs or Tits systems.

\section{$2.3 \quad B N$-pair or Tits system, and buildings}

The procedure of constructing a building from parabolic subgroups can be formalized as $B N$-pairs or Tits systems (see [Br1, p. 110]).

Definition 2.3.1 A $B N$-pair in a group $G$ consists of two subgroups $B, N$ satisfying the following properties:

1. $B, N$ generate $G$.

2. The subgroup $H=B \cap N$ is normal in $N$.

3. The quotient group $W=N / H$ is called the Weyl group of the $B N$-pair and has a generating set $S$ such that

(a) for any $s \in S, s B s^{-1} \neq B$, 
(b) for any $s \in S$ and $w \in W, B s B B w B \subset B w B \cup B s w B$.

We note that the above sets $s B s^{-1}, B w B$ etc. are well-defined even though $s, w$ are elements in the quotient group $W$. It can be shown that elements in $S$ are of order 2. For any subset $S^{\prime} \subset S$, let $\left\langle S^{\prime}\right\rangle$ be the subgroup of $W$ generated by $W$. Then $B\left\langle S^{\prime}\right\rangle B$ is a subgroup of $G$ (see [Br1, Chap $\mathrm{V}])$.

In the above example of an algebraic group $\mathbf{G}$ defined over $k$, let $\mathbf{N}$ be the normalizer of $\mathbf{T}$ and $\mathbf{B}$ a minimal $k$-parabolic subgroup of $\mathbf{G}$ which contains $\mathbf{T}$. Then $B=\mathbf{B}(k)$ and $N=\mathbf{N}(k)$ form a $B N$-pair in $\mathbf{G}(k)$.

Given a $B N$-pair, assume that the Weyl group $W$ is finite. We can construct a spherical building $\Delta$ as follows. For any subset $S^{\prime} \subset S$, the corresponding subgroup $B\left\langle S^{\prime}\right\rangle B$ is called a standard parabolic subgroup of $G$ with respect to the Tits system $(B, N)$. A subgroup of $G$ is called a parabolic subgroup if it is conjugate to a standard one. The building $\Delta$ associated with the $B N$-pair is the simplicial complex whose simplexes correspond to parabolic subgroups and the incidence relation between the simplexes is the opposite of the inclusion relation of parabolic subgroups. The set of simplexes corresponding to $B\left\langle S^{\prime}\right\rangle B$, where $S^{\prime} \subset S$, form an apartment $\Sigma$ of $\Delta$, and the translates $g \Sigma$ under $g \in G$ forms a system of apartments. The rank of the building is equal to the number of elements in $S$.

For a building $\Delta$ satisfying some conditions, it can be shown that it is isomorphic to the bulding associated with a $B N$-pair in its automorphism group. In fact, fix a labeling of the building $\Delta$ (see [Br1, p. 78, Proposition 1], also [Br1, p. 30, p. 72] and $\S 2.7$ below). Then the labeling of the vertices gives types to simplexes in $\Delta$. Let $G$ be an automorphism group of $\Delta$ that preserves the type of simplexes and the apartment system. The action is called strongly transitive if $G$ acts transitively on the set of pairs $(\Sigma, C)$, where $\Sigma$ is an apartment and $C$ is a chamber in $\Sigma$.

Assume that $G$ acts strongly transitively on $\Delta$. Define

$$
B=\{g \in G \mid g C=C\}, \quad N=\{g \in G \mid g \Sigma=\Sigma\} .
$$

Then

$$
H=B \cap N=\{g \in G \mid g \text { fixes } \Sigma \text { pointwise. }\}
$$

Then it can be shown that $B, N$ form a $B N$-pair in $G$ [Br1, Chap V].

\subsection{Rigidity of Tits buildings}

An important result of Tits [Ti2, 6.3, 6.13, 8.4.5, 9.1, 10.2] gives a classification of irreducible thick spherical buildings of rank at least 3. For completeness, we follow [Sca, §7.3] to state a sample theorem from the classification results.

Theorem 2.4.1 Every thick spherical Tits building of type $\mathbf{A}_{n}, n \geq 3$, is isomorphic to the flag complex of a projective space.

Every spherical Tits building of type $\mathbf{D}_{n}, n \geq 4, \mathbf{E}_{6}, \mathbf{E}_{7}, \mathbf{E}_{8}$ is the building of the natural BNpair (or Tits system) of the K-rational points of the split algebraic group (Chevalley) whose Dynkin diagram is of the corresponding type, where $K$ is a unique commutative field.

Every spherical Tits building of type $\mathbf{C}_{n}, n \geq 4$, is the flag complex of totally isotropic subspaces with respect to a nondegenerate trace valued Hermitian form or a nondegenerate (pseudo-)quadratic form.

The classification of buildings of higher rank is responsible for several rigidity results, for example, characterizations and classification of some finite simple groups of Lie type (see [Su1] [Su2] [Su3] 
[Har], [Car2]), rank rigidity of manifolds of nonpositive curvature [BuS1] which uses a generalization of Theorem 2.4.1 above (see Theorem 2.7.5 and $\S 2.9$ below), and classification of isoparametric submanifolds in $\mathbb{R}^{n}$ (see [Ter1] [Tho2] and $\S 2.10$ ). Though the applications are different, the strategy is the same and consists of two steps:

1. Construct a building or $B N$-pair from the given groups or manifolds.

2. Apply the classification result to determine the building and use it to recover the original object.

Another important result of Tits shows that group structures can be fully recovered from the buildings through description of morphisms between them [Ti2, Theorem 5.8] [Mos, Theorem 16.1, Corollary 16.2].

Theorem 2.4.2 Let $\mathbf{G}, \mathbf{G}^{\prime}$ be linear semisimple groups defined over $\mathbb{R}$ which have no center and no simple factors of $\mathbb{R}$-rank less than or equal to 1 . Then any isomorphism between the spherical Tits buildings $\Delta(\mathbf{G})$ and $\Delta\left(\mathbf{G}^{\prime}\right)$ induces an isomorphism between $\mathbf{G}(\mathbb{R})$ and $\mathbf{G}^{\prime}(\mathbb{R})$ as abstract groups.

This shows that the building $\Delta(\mathbf{G})$ determines the group $G=\mathbf{G}(\mathbb{R})$. Since parabolic subgroups of $G$ describe the structures of $G$ at infinity or large scale geometry, this also reflects some rigidity property of $G$. In fact, this will be used crucially in the proof of the Mostow strong rigidity in [Mos] (see Theorem 2.8.1).

Remark 2.4.3 It should be emphasized that the building $\Delta(\mathbf{G})$ does not necessarily determine $G$ as a Lie group. In fact, by Theorem $2.4 .2, \mathbf{G}(\mathbb{R})$ is isomorphic to $\mathbf{G}^{\prime}(\mathbb{R})$ as abstract groups. On the other hand, if we enrich the spherical Tits buildings to topological spherical Tits buildings as in [BuS2] (see 2.7.1 below), we can recover the topologies of $G$ and $G^{\prime}$ and should be able to show that they are isomorphic as Lie groups. This is the reason that we emphasize topological buildings in this paper. As mentioned in the introduction, the idea of topological buildings was used implicitly in the proof of the Mostow strong rigidity since the homeomorphism on the maximal Furstenberg boundaries is crucial to the proof.

\subsection{Geodesic compactification of symmetric spaces}

Let $G$ be a linear semisimple Lie group, $K \subset G$ a maximal compact subgroup, and $X=G / K$ the associated Riemannian symmetric space. For simplicity, we endow $X$ with the invariant metric induced from the Killing form. In this subsection, we will realize the spherical Tits building $\Delta(G)$ using the asymptotic geometry of $X$ and show how it can in turn be used to understand the geometry at infinity of $X$ and compactifications of $X$.

It is known that $X$ is a Riemannian symmetric space of noncompact type and hence is simply connected and of nonpositive sectional curvature, i.e., a Hadamard manifold. It admits the geodesic compactification $X \cup X(\infty)$ which is defined as follows.

All geodesics considered in this paper are of unit speed and directed. Two geodesics $\gamma_{1}(t), \gamma_{2}(t)$, $t \in \mathbb{R}$, in $X$ are called equivalent if

$$
\limsup _{t \rightarrow+\infty} d\left(\gamma_{1}(t), \gamma_{2}(t)\right)<+\infty
$$

Denote the set of equivalence classes of geodesics by $X(\infty)$. 
Let $x_{0} \in X$ be a basepoint in $X$. Then it can be shown that in each equivalence class, there is a unique geodesic passing through $x_{0}$, and hence $X(\infty)$ can be identified with the unit sphere in the tangent space $T_{x_{0}} X$. Due to this identification, $X(\infty)$ is often called the sphere at infinity.

The topology of the compactification $X \cup X(\infty)$ is defined such that an unbounded sequence $y_{j}$ in $X$ converges to an equivalence class $\xi \in X(\infty)$ if the sequence of geodesics $\gamma_{j}$ passing through $x_{0}$ and $y_{j}$ converges to a geodesic $\gamma_{\infty}$ which belongs to $\xi$.

If we identify $X$ with the open unit ball in $T_{x_{0}} X$ by the exponential map and shrinking along rays from the origin, then $X \cup X(\infty)$ is homeomorphic to the closed unit ball in $T_{x_{0}} X$. But the intrinsic definition of the compactification $X \cup X(\infty)$ allows us to see that any isometry on $X$ extends continuously to $X \cup X(\infty)$. Hence we have

Proposition 2.5.1 The G-action on $X$ extends continuously to $X \cup X(\infty)$.

This action allows one to understand parabolic subgroups of $G$ geometrically.

Proposition 2.5.2 For any point $\xi \in X(\infty)$, its stabilizer $G_{\xi}=\{g \in G \mid g \xi=\xi\}$ is a parabolic subgroup. Conversely, any (proper) parabolic subgroup $P$ fixes some boundary point in $X(\infty)$.

For proofs, see [GJT] [BJ1] [BGS]. For each (proper) parabolic subgroup $P$, let $\sigma_{P}$ be the set of points in $X(\infty)$ whose stabilizer in $G$ is exactly equal to $P$. It can be shown that the set of points in $X(\infty)$ fixed by $P$ is exactly equal to the closure of $\sigma_{P}$. Unless $P$ is a maximal proper parabolic subgroup, $\sigma_{P}$ is not closed.

Proposition 2.5.3 For any two parabolic subgroups $P_{1}, P_{2}, \sigma_{P_{1}}^{o}$ and $\sigma_{P_{2}}^{o}$ are either disjoint or identical. Furthermore, $\sigma_{P_{1}}$ is contained in the closure of $\sigma_{P_{2}}$ in $X(\infty)$ if and only if $P_{1}$ contains $P_{2}$; and $\sigma_{P}$ consists of a point if and only if $P$ is a maximal parabolic subgroup; and each $\sigma_{P}$ has a natural (spherical) simplicial structure under the identification of $X(\infty)$ with the unit sphere in $T_{x_{0}}(X)$.

See [BJ1, §I.2] for a proof. An immediately corollary of the above proposition is

Proposition 2.5.4 The disjoint decomposition $X(\infty)=\cup_{P} \sigma_{P}$ gives a simplicial complex isomorphic to the spherical Tits building $\Delta(G)$ of $G$.

It should be emphasized that the simplicial topology on $\Delta(G)$ is not the same as the topology of $X(\infty)$ when identified with the unit sphere in $T_{x_{0}} X$. For example, when $X$ is a symmetric space of rank $1, X(\infty)$ is a closed (real analytic) manifold, but $\Delta(G)$ is the disjoint union of points in $X(\infty)$ and hence induces the discrete topology on $X(\infty)$.

\subsection{Buildings and compactifications of symmetric spaces}

This identification of $\Delta(G)$ with the boundary $X(\infty)$ in Proposition 2.5.4 is important for several reasons:

1. Since $X(\infty)$ is defined in terms of asymptotic classes of geodesics, the building $\Delta(G)$ describes the asymptotic geometry at infinity of $X$.

2. The boundary $X(\infty)$ of the compactification $X \cup X(\infty)$ is assembled from the boundary pieces $\sigma_{P}$ where $P$ runs over parabolic subgroups of $G$. 
The conclusion in (2) suggests that if we change the boundary component $\sigma_{P}$ of the parabolic subgroup $P$, we could get other compactifications $\bar{X}$ of $X$. This is indeed the case, and hence the boundaries $\partial \bar{X}$ of the compactifications $\bar{X}$ often have a cell-complex structure related to the spherical Tits building $\Delta(G)$, or rather the boundaries are unions of boundary components parametrized by the Tits building.

To illustrate this, we discuss the maximal Satake compactification $\bar{X}_{\max }^{S}$ of $X$ to show that its boundary has a cell-complex structure dual to the spherical Tits building $\Delta(G)$ (for more details and proofs of the following discussions, see [GJT] and [BJ1]).

Let

$$
\tau: G \rightarrow S L(n, \mathbb{C})
$$

be a finite dimensional irreducible and faithful representation satisfying the condition:

$$
\tau(\theta(g))=\left(\tau(g)^{*}\right)^{-1}, \quad g \in G,
$$

where $\theta$ is the Cartan involution of $G$ associated with the maximal compact subgroup $K$. Then $\tau$ induces a $G$-equivariant embedding

$$
i_{\tau}: X=G / K \rightarrow S L(n, \mathbb{C}) / S U(n), \quad g K \mapsto \tau(g) \tau(g)^{*} .
$$

The quotient $S L(n, \mathbb{C}) / S U(n)$ is the symmetric space of positive definite Hermitian matrices of determinant 1 and is hence contained in the real vector space $\mathcal{H}_{n}$ of Hermitian $n \times n$-matrices. Let $P\left(\mathcal{H}_{n}\right)$ be the associated real projective space. By composing with $i_{\tau}$, we obtain an embedding

$$
i_{\tau}: X \rightarrow P\left(\mathcal{H}_{n}\right)
$$

and the closure of $i_{\tau}(X)$ in $P\left(\mathcal{H}_{n}\right)$ is called the Satake compactification of $X$ associated with the representation $\rho$, denoted by $\bar{X}_{\tau}^{S}$. Since $G$ acts on $\mathcal{H}_{n}$ through the representation $\rho$ and the embedding $i_{\tau}$ is $G$-equivariant, the $G$-action on $X$ extends to a continuous action on $\bar{X}_{\tau}^{S}$.

As a topological $G$-space, $\bar{X}_{\tau}^{S}$ only depends on the relative position of the highest weight $\mu_{\tau}$ of $\tau$, i.e., on the Weyl chamber face which contains $\mu_{\tau}$ as an interior point. When $\mu_{\tau}$ is generic, i.e., contained in the interior of the Weyl chamber, the compactification $\bar{X}_{\tau}^{S}$ is called the maximal Satake compactification and denoted by $\bar{X}_{\max }^{S}$.

The boundary $\partial \bar{X}_{\max }^{S}$ can be decomposed into boundary components parametrized by parabolic subgroups. For each parabolic subgroup $P$ of $G$, let $N_{P}$ be the unipotent radical of $P, A_{P}$ the split component invariant under the Cartan involution $\theta$ associated with $K$, and

$$
P=N_{P} A_{P} M_{P} \cong N_{P} \times A_{P} \times M_{P}
$$

be the Langlands decomposition of $P$. Note that the Langlands decomposition is a diffeomorphism but not a group isomorphism. For example, when $G=S L(n, \mathbb{R})$, and

$$
P=\left\{g=\left(\begin{array}{cc}
A & B \\
0 & C
\end{array}\right) \mid A \in M_{k}(\mathbb{R}), C \in M_{n-k}(\mathbb{R}), B \in M_{k \times n-k}(\mathbb{R}), g \in S L(n, \mathbb{R})\right\}
$$

then

$$
\begin{gathered}
N_{P}=\left\{\left(\begin{array}{cc}
I_{k} & B \\
0 & I_{n-k}
\end{array}\right) \mid B \in M_{k \times n-k}\right\}, \\
A_{P}=\left\{\left(\begin{array}{cc}
a I_{k} & 0 \\
0 & c I_{n-k}
\end{array}\right) \mid a, c \in \mathbb{R}^{\times}, a^{k} c^{n-k}=1,\right\}
\end{gathered}
$$




$$
M_{P}=\left\{\left(\begin{array}{cc}
A & 0 \\
0 & C
\end{array}\right) \mid \operatorname{det} A \operatorname{det} C=1\right\}
$$

Define

$$
X_{P}=M_{P} / K \cap M_{P}
$$

which is a lower dimensional symmetric space of noncompact type, called the boundary symmetric space associated with $P$. In the above example of $P$ in $S L(n, \mathbb{R})$, the boundary symmetric space is

$$
X_{P}=(S L(k, \mathbb{R}) / S O(k)) \times(S L(n-k, \mathbb{R}) / S O(n-k)) .
$$

Then as a set,

$$
\bar{X}_{\max }^{S}=X \cup \coprod_{P} X_{P}
$$

For any two parabolic subgroups $P_{1}, P_{2}, X_{P_{1}}$ is contained in the closure of $X_{P_{2}}$ if and only if $P_{1}$ is contained in $P_{2}$. Since each $X_{P}$ is a cell, the boundary $\partial \bar{X}_{\max }^{S}$ is a cell-complex dual to the spherical Tits building $\Delta(G)$ and the topology of the boundary components $X_{P}$ is determined by the topology of the topological building of $G$.

Remark 2.6.1 There are also other compactifications of $X$, for example the Martin compactification, whose boundaries have cell-complex structure of more complicated type. The relations between the Tits buildings and structures of the boundary components of compactifications already exist on closures of maximal flat totally geodesic submanifolds, called flats, in symmetric spaces. For example, the boundary of a flat in the maximal Satake compactification $\bar{X}_{\text {max }}^{S}$ is a polyhedral compactification whose boundary is dual to an apartment in the spherical Tits building. See [GJT] and [BJ1] for more details.

In $\bar{X}_{\max }^{S}$, there are only finitely many $G$-orbits corresponding to the finitely many conjugacy classes of parabolic subgroups. In fact, each boundary component is contained in a $G$-orbit as a proper subset and the boundary symmetric spaces of conjugate parabolic subgroups belong to the same $G$-orbit. The orbit for the minimal parabolic subgroups is the only closed one and is equal to $G / P_{0}$, where $P_{0}$ is a minimal parabolic subgroup.

Definition 2.6.2 Let $P_{0}$ be a minimal parabolic subgroup of $G$, the homogeneous space $G / P_{0}$ is called the maximal Furstenberg boundary of $X$ or $G$, and denoted by $\mathcal{X}_{0}$.

Remark 2.6.3 The fact that the maximal Furstenberg is contained canonically in $\bar{X}_{\max }^{S}$ is important in the proof of Mostow rigidity to be discussed below. It should be pointed out that when the rank of $X$ is greater than or equal to 2 , the sphere at infinity $X(\infty)$ (or the boundary of the geodesic compactification) contains infinitely many $G$-orbits which are isomorphic to the maximal Furstenberg boundary. The maximal Furstenberg boundary $\mathcal{X}_{0}$. is closely related to the Tits building. In fact, the set of chambers in the Tits building $\Delta(G)$ is parametrized by $\mathcal{X}_{0}$. Its canonical embedding in $\bar{X}_{\max }^{S}$ explains why the maximal Satake compactification rather than the geodesic compactification $X \cup X(\infty)$ is used in the Mostow strong rigidity below. 


\subsection{Topological spherical Tits buildings}

As explained in the previous subsection, the boundary of a compactification of the symmetric space $X$, for example, the geodesic compactification $X \cup X(\infty)$ and the maximal Satake compactification $\bar{X}_{\text {max }}^{S}$, has the structure of cell-complex closely related to the spherical Tits building of $X$.

On the other hand, the boundary of the compactification is a compact topological space. When $X=S L(2, \mathbb{R}) / S O(2)$ is the Poincare upper half plane, or equivalently the unit disc, the Tits building $\Delta(G)$ is the unit circle with the discrete topology. On the other hand, the boundary $X(\infty)$ is the unit circle with the usual topology.

The unit circle $X(\infty)$ with the usual topology is the topological spherical Tits building corresponding to the building $\Delta(G)$.

In fact, topological buildings are defined in [BuS2, Definition 1.1] as follows. Let $\Delta$ be a spherical Tits building of rank $r$. Then any chamber of $\Delta$ has $r$ vertexes. Fix a chamber $C$ and list its vertexes as $v_{1}, \cdots, v_{r}$. Then any other chamber has also a unique induced ordering of its vertexes. Similarly, the vertexes of every simplex $\sigma$ in $\Delta$ have also a well-defined ordering. For each $n=1, \cdots, r$, let $\Delta_{n}$ be the set of simplexes with $n$ vertexes. Then $\Delta_{1}$ is the set of vertexes, and $\Delta_{r}$ is the set of chambers. The above ordering of vertexes gives a well-defined map

$$
\Delta_{n} \rightarrow\left(\Delta_{1}\right)^{n}, \quad \sigma \mapsto\left(x_{1}, \cdots, x_{n}\right),
$$

where $x_{1}, \cdots, x_{n}$ are the vertexes of $\sigma$ listed according to the order.

Definition 2.7.1 A topological spherical Tits building is a spherical Tits building $\Delta$ with a Hausdorff topology on $\Delta_{1}$ such that the image of $\Delta_{n}$ in $\left(\Delta_{1}\right)^{n}$ under the map in Equation (2.7.1) is a closed subset.

In a topological building, we endow $\Delta_{n}$ with the subset topology induced from $\left(\Delta_{1}\right)^{n}$. We can also define a topological Tits building as a Tits building with a topology on every $\Delta_{n}$ such that the map in Equation (2.7.1) is an embedding with a closed image.

If the topology of a topological building is metrizable, it is called a metric building $[\mathrm{BuS} 2, \mathrm{p}$. 12]. Many naturally occurring spherical Tits buildings such as $\Delta(G)$ are metric buildings.

Identify $X(\infty)$ with the unit sphere in $T_{x_{0}} X$ and denote the subset distance induced from the Riemannian norm on $T_{x_{0}} X$ by $d$. Then $d$ induces the Hausdorff distance on the collection of subsets of $X(\infty)$ by:

$$
d_{H}(A, B)=\inf \left\{\delta \mid A \subseteq N_{\delta}(B), B \subseteq N_{\delta}(A)\right\},
$$

where $N_{\delta}(B)$ is the $\delta$-neighborhood of $B$.

Realize $\Delta(G)$ as a simplicial complex on $X(\infty)$. Then the Hausdorff distance function $d_{H}$ defines a metric on $\Delta_{1}, \cdots, \Delta_{r}$, where $r$ is the rank of $G$ or $\Delta(G)$. It can be checked easily that with this metric on $\Delta_{1}, \Delta(G)$ is a metric spherical Tits building and hence is a topological spherical Tits building.

Definition 2.7.2 A topological spherical Tits building $\Delta$ of rank $r$ is called compact, connected, locally connected if the topological space $\Delta_{r}$ has the corresponding property. An automorphism of a topological Tits building is an automorphism of the building whose restriction to each $\Delta_{n}$, $n=1, \cdots, r$, is a homeomorphism.

Remark 2.7.3 On a topological building $\Delta$, we can put a topology on the whole underlying space of the building $\Delta$ by gluing up the topologies on the subspaces $\Delta_{n}, n=1, \cdots, r$. Then an 
automorphism is a homeomorphism that preserves the simplicial structure. Other notions such as compactness can also be defined directly in terms of $\Delta$. This might be more useful for the compactification of the Bruhat-Tits buildings by the spherical Tits buildings in [BS1] (see also $§ 3.5$ below), since the spherical Tits buildings are topological in the sense here.

Two important results on topological buildings in [BuS2] are given in the next two theorems.

Theorem 2.7.4 If $\Delta$ is an irreducible compact metric building of rank at least 2 , then its topological automorphism group is locally compact in the compact open topology.

Before stating the next result, we need the notion of Moufang buildings. For any apartment $\Sigma \subset \Delta$, a root hyperplane in $\Sigma$ divides it into two half planes, often called roots. For a half plane $A$, let $U_{A}$ be the group consisting of all elements of $G=\operatorname{Aut}(\Delta)$ that fix all the chambers in $A$. Then $\Delta$ is called Moufang if $U_{A}$ acts transitively on the set of all apartments containing $A$, in particular $\Delta$ has abundant automorphisms [BuS2, p.22]. This Moufang condition is slightly weaker than the usual one in [Ti2] and [Ro2], but easier to state and sufficient for the next result.

Theorem 2.7.5 If $\Delta$ is an infinite, irreducible, locally connected, compact, metric Moufang spherical building of rank at least 2, then the identity component $G^{0}$ of the topological automorphism group of $\Delta$ is a simple noncompact real Lie group without center, and $\Delta$ is isomorphic to the topological spherical Tits building $\Delta\left(G^{0}\right)$.

This theorem is similar to Theorem 2.4.1. In fact, in Theorem 2.4.1, the building is required to be of rank at least three, which together with the other conditions there implies that the building is Moufang. Hence the conditions in Theorem 2.7.5 of rank being at least 2 and of being Moufang is weaker. This weakening to rank at least 2 is crucial for application to the rank rigidity of manifolds of nonpositive curvature in [BuS1] (see $\S 2.9$ below).

\subsection{Mostow strong rigidity}

The fundamental result of Mostow rigidity [Mos] concerns the rigidity of finite volume locally symmetric spaces of higher rank. It says roughly that the fundamental group determines the space isometrically up to scaling factors. This is a major application of the result of Tits on rigidity (or classification) of spherical Tits buildings of higher rank.

Let $G$ be a connected semisimple Lie group without center and with no nontrivial compact factor, $K \subset G$ a maximal compact subgroup, and $X=G / K$ the associated symmetric space as before. Any torsion free discrete subgroup $\Gamma$ of $G$ acts freely and properly on $X$, and the quotient $\Gamma \backslash X$ is a smooth manifold, called a locally symmetric space.

The discrete subgroup $\Gamma$ is called a lattice if $\Gamma \backslash X$ has finite volume and uniform if $\Gamma \backslash X$ is compact. It is called reducible if $G$ admits a nontrivial decomposition $G=G_{1} \times G_{2}$, which implies an isometric decomposition $X=X_{1} \times X_{2}$, and two subgroups $\Gamma_{1} \subset G_{1}, \Gamma_{2} \subset G_{2}$ such that $\Gamma$ is commensurable to $\Gamma_{1} \times \Gamma_{2}$, i.e., $\Gamma \cap \Gamma_{1} \times \Gamma_{2}$ has finite index in both $\Gamma$ and $\Gamma_{1} \times \Gamma_{2}$. In this case, after lifting to a finite cover, $\Gamma \backslash X$ splits as a product of two locally symmetric spaces. An equivalent definition is that the images of $\Gamma$ in $G_{1}, G_{2}$ are discrete [Mos, p. 133]. If $\Gamma$ is not reducible, it is called irreducible.

We recall that a flat in $X$ is a totally geodesic flat subspace in $X$. It is known that $G$ acts transitively on the set $\mathcal{F}$ of maximal flats $X$, and hence the maximal flats in $X$ have the same dimension, which is equal to the rank of $G$. For convenience, a flat means a maximal flat in $X$ in the following unless indicated otherwise.

The precise statement of the Mostow strong rigidity [Mos] is given in the following theorem. 
Theorem 2.8.1 Let $X=G / K$ be a symmetric space of noncompact type as above, and $\Gamma$ an irreducible uniform lattice in $G$. Assume that either the rank of $X$ is at least 2, or the rank of $X$ is equal to 1 and $\operatorname{dim} X \geq 3$. Suppose that $X^{\prime}=G^{\prime} / K^{\prime}$ is a symmetric space of noncompact type and $\Gamma^{\prime}$ is a uniform lattice acting on $X^{\prime}$. If $\Gamma^{\prime}$ is isomorphic to $\Gamma$ (as abstract groups), then $\Gamma \backslash X$ and $\Gamma^{\prime} \backslash X^{\prime}$ are isometric up to suitable normalizing scalars of the irreducible factors of $X$, which implies that $G$ and $G^{\prime}$ are isomorphic.

This theorem says that under the conditions in the theorem, the fundamental group of $\Gamma \backslash X$ determines $\Gamma \backslash X$ isometrically up to suitable scaling. It should be pointed out that both conditions are necessary. For example, if $G=S L(2, \mathbb{R}), X=S L(2, \mathbb{R}) / S O(2)$, the upper half plane, the rank of $X$ is equal to 1 , and the strong rigidity of $\Gamma$ fails due to the high dimension of the moduli spaces of Riemann surfaces (or the deformation space of $\Gamma \backslash X)$. Now take $G=S L(2, \mathbb{R}) \times S L(2, \mathbb{R}$ ), the associated symmetric space $X$ has rank equal to 2 . Take a reducible lattice $\Gamma=\Gamma_{1} \times \Gamma_{2}$, where $\Gamma_{1}, \Gamma_{2} \subset S L(2, \mathbb{R})$ are uniform lattices. Due to the failure of the strong rigidity for $\Gamma_{i}$, it also fails for such reducible $\Gamma$.

Remark 2.8.2 For a history of the results leading to the Mostow strong rigidity, see [Mos, p. 5]. A generalization of this theorem is given in [BGS]. In fact, when the rank of $X$ is at least 2, the target manifold $\Gamma^{\prime} \backslash X^{\prime}$ can be replaced by any compact manifold with nonpositive sectional curvature. The assumption on the rank is necessary due to existence of exotic negatively curved metrics on locally symmetric spaces of rank 1 (see [On] [FJO] [AF]). See [Sp1] [Sp2] for surveys of other aspects of rigidity results, in particular, from the point of view of dynamic systems, which is a huge area.

In the case of rank at least 2, this theorem is proved using the result in Theorem 2.4.2 on Tits buildings. In the case of rank 1, it uses generalizations of quasi-conformal maps on the sphere at infinity $X(\infty)$. See [Sp2] [GP] for an outline of the proof in the case of real hyperbolic spaces. See [Iv] for related results.

Since the rank 1 case does not make use of buildings, we will only briefly outline the proof for the case of higher rank.

The proof in [Mos] proceeds in the following steps:

1. Since $X, X^{\prime}$ are contractible, $\Gamma \backslash X$ and $\Gamma^{\prime} \backslash X^{\prime}$ are $K(\Gamma, 1)$-spaces. The isomorphism between $\Gamma$ and $\Gamma^{\prime}$ induces a homotopy equivalence

$$
\psi: \Gamma \backslash X \rightarrow \Gamma^{\prime} \backslash X^{\prime}
$$

which induces a $\Gamma$-equivariant quasi-isometry

$$
\varphi: X \rightarrow X^{\prime}
$$

2. Let $\mathcal{X}_{0}, \mathcal{X}_{0}^{\prime}$ be the maximal Furstenberg boundaries sitting in the boundary of the maximal Satake compactifications $\bar{X}_{\max }^{S}$ and ${\overline{X^{\prime}}}_{\max }^{S}$. The quasi-isometry $\varphi$ induces a homeomorphism

$$
\varphi_{0}: \mathcal{X}_{0} \rightarrow \mathcal{X}_{0}^{\prime}
$$

which is called the boundary value map of $\varphi$.

3. The same proof as in (2) shows that $\varphi$ induces an isomorphism between the spherical Tits buildings $\Delta(G)$ and $\Delta\left(G^{\prime}\right)$. 
4. Apply Theorem 2.4.2 to conclude $G \cong G^{\prime}$ as abstract groups. Combining with (2), it implies that $G$ and $G^{\prime}$ are isomorphic as Lie groups.

Recall that a map $\varphi: X \rightarrow X^{\prime}$ is called a quasi-isometry if there exist positive constants $L, C$ such that for all $x_{1}, x_{2} \in X$,

$$
L^{-1} d\left(x_{1}, x_{2}\right)-C \leq d^{\prime}\left(\varphi\left(x_{1}\right), \varphi\left(x_{2}\right)\right) \leq L d\left(x_{1}, x_{2}\right)+C,
$$

where $d, d^{\prime}$ are the distance functions of $X$ and $X^{\prime}$ respectively, and for every $x^{\prime} \in X^{\prime}$,

$$
d^{\prime}\left(x^{\prime}, \varphi(X)\right) \leq C
$$

Then Step (1) basically follows from the assumption that $\Gamma \backslash X$ and $\Gamma^{\prime} \backslash X^{\prime}$ are compact and $X, X^{\prime}$ are contractible.

The proof of Step (2) depends on the identification of the maximal Furstenberg boundary $\mathcal{X}_{0}$ with the set of equivalence classes of Weyl chambers in flats $\mathcal{F}$ in $X$.

Specifically, for any basepoint $x_{0} \in X$, any flat $F$ in $X$ passing through $x_{0}$ is of the form $e^{\mathfrak{a}} x_{0}$, where $\mathfrak{a}$ is a maximal abelian subalgebra in $\mathfrak{p}_{x_{0}}$, where $\mathfrak{p}_{x_{0}}$ is the component invariant under $\theta_{0}$ in the Cartan decomposition $\mathfrak{g}=\mathfrak{k}_{x_{0}}+\mathfrak{p}_{x_{0}}$, where $\theta_{0}$ is the Cartan involution $\theta_{0}$ associated with $x_{0}$. The connected components of the complement of the root hyperplanes in $\mathfrak{a}$ are called Weyl chambers. For any chamber $C$ of $\mathfrak{a}$, the image $e^{C} x_{0}$ in $X$ is called a chamber in $X$, which is also denoted, for simplicity, by $C$. Two chambers $C_{1}, C_{2}$ are called equivalent (or asymptotic) if

$$
d_{H}\left(C_{1}, C_{2}\right)<+\infty
$$

where $d_{H}$ is the Hausdorff distance on subsets of $X$. For each chamber $C$, denote its equivalence class by $[C]$. Then the set of equivalence classes of Weyl chambers $[C]$ can naturally be identified with the maximal Furstenberg boundary $\mathcal{X}_{0}$ [Mos, Lemma 4.1]. The identification comes from the following facts:

1. For any chamber $C$ in $X$ and a sequence $x_{j}$ in $C$ which diverges to infinity and its distance to the chamber walls also goes to infinity, then $x_{j}$ converges to a point $\xi \in \mathcal{X}_{0}$. Hence, there is a unique point in $\mathcal{X}_{0}$ corresponding to each chamber $C$.

2. If two chambers are equivalent, then they correspond to the same point.

To prove the existence of the boundary value map $\varphi_{0}$, it suffices to show that for any chamber $C$ in $X$, there is a chamber $C^{\prime}$ in $X$ and hence a unique equivalence class $\left[C^{\prime}\right]$ of chambers such that

$$
d_{H}\left(C^{\prime}, \varphi(C)\right)<+\infty
$$

Then we define the map $\varphi_{0}$ by

$$
\varphi_{0}([C])=\left[C^{\prime}\right]
$$

For this purpose, a crucial role is played by behaviors of flats under quasi-isometries. First we note that the rank of $X$ can be characterized algebraically in terms of $\Gamma$ [Mos, Lemma 11.3].

Proposition 2.8.3 Under the assumption of Theorem 2.8.1, the rank of $G$ and hence of $X$ is equal to the maximal rank of abelian subgroups of $\Gamma$. Hence, the rank of $X$ is equal to the rank of $X^{\prime}$.

Let $\mathcal{F}^{\prime}$ be the set of flats in $X^{\prime}$. 
Proposition 2.8.4 Let $\varphi: X \rightarrow X^{\prime}$ be the $\Gamma, \Gamma^{\prime}$-equivariant quasi-isometry constructed in Equation 2.8.2. Then for any flat $F \in \mathcal{F}$, there is a unique flat $F^{\prime} \in \mathcal{F}^{\prime}$ such that

$$
d_{H}\left(F^{\prime}, \varphi(F)\right)<+\infty .
$$

Define the map

$$
\bar{\varphi}: \mathcal{F} \rightarrow \mathcal{F}^{\prime}, \quad F \mapsto F^{\prime}
$$

Then $\bar{\varphi}$ is a homeomorphism.

This is proved in [Mos, Lemma 14.1]. To apply this proposition to prove the chamber approximation in Equation 2.8.6, we need to characterize chambers in $X$ in terms of asymptotic intersection of flats in the geodesic compactification $X \cup X(\infty)$. In fact, Mostow introduced splices in [Mos, p. 107] in terms of asymptotic intersection of flats $F_{0} \cap_{x} F$ [Mos, p. 56], where $x \in F$ and

$$
F_{0} \cap_{x} F=\cup\left\{\text { rays in } F \text { starting from } x_{0} \text { and contained in a bounded neighborhood of } F_{0}\right\} \text {. }
$$

It can be seen that Weyl chambers and their faces are splices. Then he introduced the notion of irreducible splices and showed that they are given by chambers and chamber faces.

Remark 2.8.5 By definition, $F_{0} \cap_{x} F$ clearly depends on the choice of the basepoint $x$. On the other hand, its boundary points in the geodesic compactification $X \cup X(\infty)$, i.e., the intersection

$$
\overline{F_{0} \cap_{x} F} \cap X(\infty)
$$

is independent of $x$, where $\overline{F_{0} \cap_{x} F}$ is the closure of $F_{0} \cap_{x} F$ in $X \cup X(\infty)$. Furthermore, this intersection with $X(\infty)$ can be shown to be equal to the intersection

$$
\overline{F_{0}} \cap \bar{F} \cap X(\infty),
$$

and determines $F_{0} \cap_{x} F$. For this reason, we call $F_{0} \cap_{x} F$ the asymptotic intersection of the flats $F_{0}$ and $F$.

Combining Proposition 2.8.4 with the observation that Weyl chambers and their faces are given by irreducible splices, Mostow proved [Mos, Theorem 15.2] the following result.

Proposition 2.8.6 Given any chamber $C$ in $X$, there exists a chamber $C^{\prime}$ in $X^{\prime}$ such that

$$
d_{H}\left(C^{\prime}, \varphi(C)\right)<+\infty
$$

and hence there is a well-defined map

$$
\varphi_{0}: \mathcal{F} \rightarrow \mathcal{F}_{0}^{\prime}, \quad[C] \rightarrow\left[C^{\prime}\right],
$$

which can be shown to be a homeomorphism.

In fact, the same proof works for chamber faces and show that

Proposition 2.8.7 For any Weyl chamber face $\sigma_{C}$ in $X$, there exists a chamber face $\sigma_{C^{\prime}}$ of the same dimension in $X^{\prime}$ such that

$$
d_{H}\left(\sigma_{C^{\prime}}, \varphi\left(\sigma_{C}\right)\right)<+\infty
$$


As in the case of chambers, we can also define an equivalence relation on chamber faces. Two chamber faces $\sigma_{1}, \sigma_{2}$ in $X$ are called equivalent if

$$
d_{H}\left(\sigma_{1}, \sigma_{2}\right)<+\infty
$$

Clearly two equivalent chamber faces have the same dimension. Define an incidence relation on the set of equivalence classes of chamber faces $[\sigma]$ as follows: an equivalence class $\left[\sigma_{1}\right]$ is called a face of $\left[\sigma_{2}\right]$ if there are are representatives $\sigma_{1} \in\left[\sigma_{1}\right]$ and $\sigma_{2} \in\left[\sigma_{2}\right]$ such that $\sigma_{1}$ is a face of $\sigma_{2}$.

It can be shown that the simplicial complex with simplexes corresponding to the equivalence classes of chamber faces and the incidence relation defined here is the spherical Tits building $\Delta(G)$. Then an immediate corollary is the following:

Corollary 2.8.8 The equivariant map $\varphi: X \rightarrow X^{\prime}$ induces an isomorphism between $\Delta(G) \rightarrow$ $\Delta\left(G^{\prime}\right)$.

To finish the proof of Theorem 2.8.1, there are two cases to consider depending on whether $G$ has any simple factor of rank equal to 1 or not.

Suppose that $G$ has no simple factor of rank 1. Then Theorem 2.4.2 implies that $G$ and $G^{\prime}$ are isomorphic as abstract groups. Since the maximal Furstenberg boundaries $\mathcal{X}_{0}, \mathcal{X}_{0}^{\prime}$ are included canonically in $\Delta(G)$ and $\Delta\left(G^{\prime}\right)$ and are stable under the action of $G$ and $G^{\prime}$ respectively and the maps $G \rightarrow \operatorname{Aut}\left(\mathcal{X}_{0}\right), G^{\prime} \rightarrow \operatorname{Aut}\left(\mathcal{X}_{0}^{\prime}\right)$ are injective, we can use the fact that $\varphi_{0}: \mathcal{X}_{0} \rightarrow \mathcal{X}_{0}^{\prime}$ is a homeomorphism (Proposition 2.8.6) to show that $G$ and $G^{\prime}$ are isomorphic as Lie groups, which proves Theorem 2.8.1 in this case.

In the second case, $G$ is reducible and contains simple factors of rank 1 . The last part of the arguments is different from above and given in [Mos, $\S 18$, Corollary 18.2]. The basic idea is as follows. Suppose $G$ has two simple factors, $G=G_{1} \times G_{2}$. Then the isomorphism of the Tits buildings in Proposition 2.8.8 implies that $G^{\prime}$ has a similar decomposition $G^{\prime}=G_{1}^{\prime} \times G_{2}^{\prime}$. Let $\pi_{i}: G \rightarrow G_{i}$ be the projection to the factors, $i=1$, 2 . Since $\Gamma$ is irreducible, $\pi_{i}(\Gamma)$ is dense in $G_{i}$, and hence the isomorphism of $\pi_{i}(\Gamma)$ and $\pi_{i}\left(\Gamma^{\prime}\right)$ via the restriction $\varphi_{i}$ of $\varphi_{0}$ implies that $G_{i}$ is isomorphic to $G_{i}^{\prime}$ as Lie groups, which completes the proof of Theorem 2.8.1 in this case.

Remark 2.8.9 In both cases, the existence of the boundary value map $\varphi_{0}$ and the fact that it is a homeomorphism is crucial in the proof of Theorem 2.8.1. In this case, the homeomorphism (or rather isomorphism) $\varphi_{0}$ is induced from the quasi-isometry $\varphi$. This confirms the philosophy that when one goes to infinity, finite (or small) ambiguities are smoothed out (or ignored) and one gets nicer maps.

In the proof both the fact that $\varphi$ induces an isomorphism of the Tits buildings and the fact that the boundary value map $\varphi_{0}$ is a homeomorphism are used. If one uses the topological spherical buildings of the Lie groups $G$ and $G^{\prime}$, then $\varphi$ induces an isomorphism of the topological spherical buildings, and arguments similar to those in [BuS2] should imply $G$ and $G^{\prime}$ are isomorphic as Lie groups as in Theorem 2.4.2.

\subsection{Rank rigidity of manifolds of nonpositive curvature}

In a list of open problems in [Yau2], Yau raised the following question, Problem 65 (only a part of the problem is stated here in a slightly modified form):

Define the rank of manifolds of nonpositive curvature so that it agrees with the standard one for locally symmetric spaces and describe the rigidity of such nonpositively curved manifolds when the rank is at least two. 
This question has generated a lot of work on nonpositively curved manifolds and was solved (see [Ba1] [BuS1] [Ba3] [BBE] [BBS], and [Leb] for some generalizations). The solution in [BuS1] uses classification of topological Tits buildings in Theorem 2.7.5. We briefly recall the precise formulation of the problem and its proof in [BuS1].

Let $M$ be a complete Riemannian manifold of nonpositive (sectional) curvature. Let $S M$ be the unit sphere subbundle in the tangent bundle of $M$. For each $v \in S M$, let $\gamma_{v}$ be the geodesic in $M$ which has the initial velocity vector $v$. Define the rank of $v$, denoted by $\operatorname{rank}(v)$, to be the dimension of the space of parallel Jacobi fields along the geodesic $\gamma_{v}$. We remark that $\operatorname{rank}(v)$ measures the maximal dimension of infinitesimal flat containing $\gamma_{v}$. In fact, the variation of a family of geodesics gives a Jacobi field. If the family of geodesics is parallel, the Jacobi field is parallel.

Define

$$
\operatorname{rank}(M)=\min \{\operatorname{rank}(v) \mid v \in S M\} .
$$

For any $v \in S M$, the tangent vector of $\gamma_{v}$ is a parallel Jacobi field, and hence $\operatorname{rank}(v) \geq 1$, which implies that

$$
\operatorname{rank}(M) \geq 1 \text {. }
$$

Let $\tilde{M}$ be the universal covering space of $M$. If $\tilde{M}$ is a symmetric space of noncompact type of rank $r$, then $M$ is nonpositively curved. It can be shown that the $\operatorname{rank}(M)$ as defined here is also equal to $r$. In fact, it is realized when $v$ is a regular tangent vector. Hence, the definition of rank here is a correct generalization for all nonpositively curved manifolds, and solves the first part of Yau's problem.

The second part of Yau's problem on the rank rigidity was solved in [Ba3] and [BuS1].

Theorem 2.9.1 Suppose that $M$ is nonpositively curved and has finite volume. Then its universal covering space $\tilde{M}$ is a space of rank 1 , or a symmetric space of noncompact type, or a Euclidean space, or a product of such spaces.

The manifold $M$ is called irreducible if there is no finite cover of $M$ which splits isometrically as a nontrivial product. This is related to the notion of irreducible lattices introduced before Theorem 2.8.1. It should be emphasized that $\tilde{M}$ could split.

A corollary of the above theorem is the following.

Corollary 2.9.2 Suppose that $M$ is nonpositively curved and has finite volume. If $M$ is irreducible and $\operatorname{rank}(M) \geq 2$, then $M$ is a locally symmetric space of noncompact type.

The basic idea of the proof in [BuS1] is as follows. Weyl simplexes in $\tilde{M}$ are introduced using notions of regular tangent vectors. Basically, a vector is regular if it is tangent to a unique $r$ dimensional flat, where $r=\operatorname{rank}(M)$. A related weaker notion of $p$-regular was also introduced [BBS, Definition 2.1] and $\ell$-regular in [BuS1, §2]. Then Weyl simplexes at infinity are defined [BuS1, Definition 2.2] and are shown to form a compact, metric, locally connected topological spherical Tits building of rank equal to $\operatorname{rank}(M)$, denoted by $\Delta(\tilde{M})$ [BuS1, Proposition 3.12]. (Note that the dimension of $\Delta(\tilde{M})$ is equal to $\operatorname{rank}(M)-1$.) Furthermore, it is Moufang [BuS1, Proposition 3.15]. If $\tilde{M}$ is irreducible, the building $\Delta(\tilde{M})$ is also irreducible [BuS1, Theorem 4.1].

To prove Theorem 2.9.1, it suffices to consider the case that $M$ is irreducible and $\operatorname{rank}(M) \geq 2$. There are two cases depending on if $\tilde{M}$ is irreducible.

If $\tilde{M}$ is reducible, the rigidity was proved in [Eb2, proposition 4.1]. If $\tilde{M}$ irreducible, then the topological building is irreducible, and hence by Theorem 2.7.5, the identity component $G^{0}$ of the 
topological automorphism of the topological building is a simple Lie group. Let $\Delta\left(G^{0}\right)$ be the associated topological spherical Tits building. Then it is isomorphic to the building $\Delta(\tilde{M})$.

This isomorphism was used to define a map

$$
\Phi: \tilde{M} \rightarrow X=G^{0} / K
$$

where $K \subset G^{0}$ is a maximal compact subgroup [BuS1, p. 56]. In fact, for any $p \in \tilde{M}$, the geodesic symmetry $\sigma_{p}$ of $\tilde{M}$ at $p$ defines a topological automorphism of $\Delta(\tilde{M})$, which in turn gives an involutive isomorphism $\theta_{p}$ of $G^{0}$. It can be shown that $\theta_{p}$ induces an isometry of $X$ and has a unique fixed point $q \in X$. Then define

$$
\Phi(p)=q .
$$

This idea was used earlier in the proof of a generalization of the strong Mostow rigidity by Gromov in [BGS, Chap. 4]. The last step is to show that $\Phi$ is an isometry up to a suitable multiple.

\subsection{Classification of isoparametric submanifolds}

Another application of the topological spherical Tits buildings to differential geometry concerns isoparametric submanifolds in Euclidean spaces [Tho2] [Ter1].

Isoparametric hypersurfaces in $\mathbb{R}^{n+1}$ were introduced by E.Cartan and defined to be hypersurfaces in $\mathbb{R}^{n+1}$ whose principal curvatures are constant. Clearly, affine $n$-planes and $n$-spheres in $\mathbb{R}^{n+1}$ are isoparametric. He showed that any connected isoparametric hypersurface is contained an affine $n$-plane, or $n$-sphere, or the product of a $k$-sphere with an affine $(n-k)$-plane.

Isoparametric submanifolds of higher codimension were introduced by Terng, motivated by the problem of finding submanifolds with simple local invariants. They turn out to be related to Coxeter groups and spherical Tits buildings, which reflect their global symmetries.

Definition 2.10.1 A submanifold in $\mathbb{R}^{n+r}$ of codimension $r$ is called isoparametric if its normal bundle is flat and the principal curvatures in the direction of any parallel normal vector field are constant.

See [Ter1] [Tho1] and the references there for the history and more motivations. (There is a thorough discussion about the origin of isoparametric hypersurfaces and related topics in [Tho1].)

It is known that any compact isoparametric submanifold is contained in some sphere, and hence the only compact isoparametric hypersurfaces are spheres.

Isoparametric submanifolds can be defined explicitly in terms of isoparametric maps and the associated foliation (or disjoint decomposition) as follows.

Definition 2.10.2 A smooth map $f=\left(f_{1}, \cdots, f_{r}\right): \mathbb{R}^{n+r} \rightarrow \mathbb{R}^{r}$ is called isoparametric if

1. $f$ has a regular value,

2. for any $i, j, k=1, \cdots, r,<\nabla f_{i}, \nabla f_{j}>$ and $\Delta f_{k}$ are constants on all fibers of the map,

3. for any $i, j=1, \cdots, r$, on each fiber of $f,\left[\nabla f_{i}, \nabla f_{j}\right]$ is a linear combination of $\nabla f_{1}, \cdots, \nabla f_{r}$ with constant coefficients.

For any isoparametric map $f$, the fibers of $f$ give a disjoint decomposition, called an isoparametric foliation of $\mathbb{R}^{n+r}$. It is known that the fiber over a regular value in $\mathbb{R}^{r}$ is an isoparametric submanifold of $\mathbb{R}^{n+r}$. Conversely, it is also known [Ter2] that every isoparametric submanifold occurs as a leaf in an isoparametric foliation. 
An isoparametric submanifold is called full if it is not contained in a proper affine subspace, and irreducible if it is not the product of two isoparametric submanifolds. Since a complete isoparametric submanifold is the product of a compact isoparametric submanifold with a Euclidean space, we will only discuss compact, full, and irreducible isoparametric submanifolds.

Another important class of isoparametric submanifolds arises from group spheres in the theory of symmetric spaces. Specifically, let $G$ be a noncompact semisimple Lie group and $K \subset G$ a maximal compact subgroup. Let $X=G / K$ be the associated symmetric space, and $r$ be the rank of $X$. Let

$$
\mathfrak{g}=\mathfrak{k}+\mathfrak{p}
$$

be the Cartan decomposition of $\mathfrak{g}$ determined by the Cartan involution $\theta$ corresponding to $K$. Then the tangent space $T_{x_{0}} X$ of $X$ at the basepoint $x_{0}=K$ can be identified with $\mathfrak{p}$. Since the adjoint action of $K$ preserves the Cartan decomposition, it restricts to the subspace $\mathfrak{p}$. This action of $K$ on $\mathfrak{p}=T_{x_{0}} X$ preserves the inner product and is called the isotropy representation of the symmetric space $X$. The $K$-orbits in $\mathfrak{p}$ give an isoparametric foliation, and the principal $K$-orbits, i.e., the orbits of maximal dimension, are isoparametric submanifolds. They are called flag manifolds. See [Ter1] for detailed discussion of their geometry as submanifolds of $\mathfrak{p}$.

Remark 2.10.3 When the rank $r$ of $X$ is equal to 1 , every nontrivial $K$-orbit is a principal orbit and is a sphere. In general, the principal $K$-orbits are of codimension $r$. Since the exponential map exp $: \mathfrak{p} \rightarrow X$ is a $K$-equivariant diffeomorphism, these $K$-orbits in $\mathfrak{p}$ can be looked upon as submanifolds in $X$. Then they are called group sphere in [GJT] for the following reason. Harmonic functions on $\mathbb{R}^{n}$ are characterized by the property that the average value on any sphere is equal to the value at the center. For the symmetric space, a function is called strongly harmonic if it is a joint eigenfunction of all invariant differential operators, which form a ring with $r$ generators. A strongly harmonic function on $X$ is characterized by the property that its average value over any group sphere is equal to its value at the center. The principal $K$-orbits can also be identified with the Poisson boundary of $X$, which is crucial for the generalization of the Poisson integral formula by Furstenberg $[\mathrm{Fu}]$.

The principal orbits of the isotropy representations of symmetric spaces are the main sources of isoparametric submanifolds in Euclidean spaces.

Proposition 2.10.4 Any homogeneous isoparametric submanifold in a Euclidean space is a principal $K$-orbit of the isotropy representation of a symmetric space $X=G / K$.

This result was proved by Palais and Terng [PT]. The next result of Thorbergsson [Tho2] removed the homogeneity assumption when the codimension is greater than or equal to 3 .

Theorem 2.10.5 Any compact irreducible, full isoparametric submanifold in a Euclidean space of codimension at least 3 is a principal orbit of the isotropy representation of a symmetric space.

It should be pointed out that the assumption on the codimension is necessary. There are many inhomogeneous examples of isoparametric submanifolds in Euclidean spaces of codimension 2. As pointed out earlier, in the case of dimension 1, compact isoparametric submanifolds are spheres. So the classification is only open in the case of codimension 2 (see [FKM], and also [Kr1] for recent progress on this problem). Theorem 2.10.5 has later been proved without using buildings in [HeL], [Ol] and [EsH] (see also [Esc1] [Esc2]). But the connection with the building is beautiful and fits in well with the themes of this survey. 
The idea of the proof of Theorem 2.10.5 is as follows. Let $M^{n}$ be a compact, full and irreducible isoparametric submanifold in $\mathbb{R}^{n+r}$, where $r \geq 3$. Assume without loss of generality that $M$ is contained in the unit sphere $S^{n+r-1}$ (with center at the origin).

1. Associate a simplicial complex $\Delta(M)$ of dimension $r$ to $M$ whose underlying space is the unit sphere $S^{n+r-1}$.

2. Show that the simplicial complex $\Delta(M)$ is a spherical Tits building of rank $r$, which is then a topological spherical building due to its realization in $S^{n+r-1}$.

3. Apply Theorem 2.7.5 on classification of topological Tits buildings to show that $\Delta(M)$ is the topological building associated with a simple Lie group $G$. In fact, the assumption $r \geq 3$ implies that the building is Moufang.

4. The symmetry of $\mathbb{R}^{n+r}$ with respect to the origin picks out a maximal compact subgroup $K$ of $G$. Define an action of $K$ on $\mathbb{R}^{n+r}$ using the fact that the underlying space of $\Delta(M)$ is the unit sphere $S^{n+r-1}$.

5. Show that the action of $K$ on $\mathbb{R}^{n+r}$ is the isotropy representation of the symmetric space $G / K$ and its principal orbits are isometric to the isoparametric submanifold $M$ up to scaling.

In this proof, the use of the topological building, rather than the usual building, is important to get Lie groups $G$ and $K$. The building is also crucial to get the desired action of $K$ on $\mathbb{R}^{n+r}$.

To motivate the construction of the simplicial complex $\Delta(M)$ in Step (1), we explain a construction of the spherical Tits building $\Delta(G)$ using the geometry of a principal $K$-orbit in the isotropy representation.

Let $H \in \mathfrak{p}$ be a regular unit vector, i.e., a unit vector contained in a unique maximal abelian subspace in $\mathfrak{p}$. Then the orbit $K \cdot H$ is a principal orbit, denoted by $\mathcal{O}$. Let $N \mathcal{O}$ be its normal bundle in $\mathfrak{p}$. Then for any $p \in \mathcal{O}$, there exists a unique maximal abelian subalgebra $\mathfrak{a}$ in $\mathfrak{p}$ which contains $p$. Furthermore, $\mathfrak{a}$ can be identified with the fiber $N_{p} \mathcal{O}$ in such a way such that the origin of the vector space $N_{p} \mathcal{O}$ corresponds to $p \in \mathfrak{a}$. In fact, since $\mathcal{O}$ is contained in the unit sphere in $\mathfrak{p}, p$ is contained in $N_{p} \mathcal{O}$; the Cartan decomposition $G=K \exp \mathfrak{a} K$ implies that $X=K \exp \mathfrak{a} \cdot x_{0}$, where $x_{0}=K \in X$, and hence the codimension of $\mathcal{O}$ is equal to $\operatorname{dim} \mathfrak{a}$, the rank of $G$ or $X$.

The Lie algebra $\mathfrak{g}$ decomposes into root spaces

$$
\mathfrak{g}=\mathfrak{g}_{0}+\sum_{\alpha \in \Sigma(\mathfrak{g}, \mathfrak{a})} \mathfrak{g}_{\alpha}
$$

where

$$
\mathfrak{g}_{\alpha}=\{Y \in \mathfrak{g} \mid[H, Y]=\alpha(H) Y, H \in \mathfrak{a}\},
$$

and $\Sigma(\mathfrak{g}, \mathfrak{a})=\left\{\alpha \in \mathfrak{a}^{*} \mid \mathfrak{g}_{\alpha} \neq 0\right\}$. Each root $\alpha \in \Sigma(\mathfrak{g}, \mathfrak{a})$ defines a root hyperplane $\mathbf{H}_{\alpha}$, and the root hyperplanes divide $\mathfrak{a}$ into Weyl chambers and chamber faces. The intersection of the unit sphere $S^{n+r-1}$ with these chambers and chamber faces determine a finite simplicial complex, the Coxeter complex associated with $G$. When $p \in \mathcal{O}$ changes, $\mathfrak{a}$ changes, and these Coxeter complexes fit together into the spherical Tits building $\Delta(G)$.

The crucial observation here is that these Coxeter complexes can be constructed from the geometry of $\mathcal{O}$ as a submanifold. In fact, the root hyperplanes $\mathbf{H}_{\alpha}$ in $\mathfrak{a}$ are exactly the intersection of $\mathfrak{a}$ with the set of focal points of $\mathcal{O} \in \mathfrak{p}$, i.e., the set of critical values of the exponential map restricted to the normal bundle $N \mathcal{O}$. In the case of rank 1, for example, $G=S O(2,1)$, $\mathcal{O}$ is the unit sphere, and the only focal point is the origin; each maximal abelian subspace $\mathfrak{a}$ has dimension 
1 , and the only root hyperplane is the origin. This example also explains the need for the shift in identifying $\mathfrak{a}$ with $N_{p} \mathcal{O}$ above. (To get a less trivial but explicit example, think of the product $S O(2,1) \times S O(2,1)$. The Weyl chamber decomposition is given by the coordinate quadrants.)

Now the construction of the simplicial complex $\Delta(M)$ in Step (1) is similar. Let $N M$ be the normal bundle of $M$ in $\mathbb{R}^{n+r}$. For each point $p \in M$, the intersection of the set of focal points of $M$ in $\mathbb{R}^{n+r}$ with the fiber $N_{p} M$, which is identified with a subspace of $\mathbb{R}^{n+r}$ by shifting by $p$, gives a collection of hyperplanes. They divide $N_{p} M$ into chambers and chamber faces, whose intersections with the unit sphere $S^{n+r-1}$ give a finite simplicial complex. When $p \in M$ changes, these finite complexes form the simplicial complex $\Delta(M)$.

To show that $\Delta(M)$ is a building is not easy. In fact, in the above construction of $\Delta(G)$ using the principal $K$-orbit $\mathcal{O}, \mathfrak{a}$ corresponds to a maximal totally geodesic subspace in $X$ passing through

the basepoint $x_{0}=K$, and its Coxeter complex is an apartment in $\Delta(G)$. They form a system of apartments in $\Delta(G)$ which is invariant under $K$ but not under $G$. This causes some difficulties in showing $\Delta(G)$ satisfies the axioms of buildings, for example, that any two chambers are contained in an apartment. In general, given two chambers in $\Delta(G)$, apartments containing the two chambers are not an apartment corresponding to a flat in $X$ passing through $x_{0}$, or corresponding to $\mathfrak{a}$.

This shows that $\Delta(M)$ is defined as the union of special apartments. Proving that $\Delta(M)$ is a spherical Tits building in Step (2) is not easy. In fact, it is the major part of the paper [Tho2], which depends on a local approach of Tits on constructing buildings.

Remark 2.10.6 Though isoparametric submanifolds of codimension 2 have not been classified, many known examples are connected to rank-2 buildings. In fact, in $[\mathrm{FKM}]$, an isoparametric hypersurface in a sphere with precisely four principal curvatures was constructed for every real representation of a Clifford algebra. It is proved in [Tho3] that each of these hypersurfaces is the flag manifold of a (differentiable) polar plane, i.e., a building of type $C_{2}$, also called a generalized quadrangle. Most of these polar planes are not Moufang buildings, and hence Theorem 2.7.5 does not apply. In fact, they do not come from isotropy representations of symmetric spaces. See [Kr1] for related results on isoparametric submanifolds of codimension 2. Together with Theorem 2.10.5, this result shows that all presently known isoparametric submanifolds are associated with buildings.

\subsection{Spherical buildings and compactifications of locally symmetric spaces}

Let $\mathbf{G} \subset G L(n, \mathbb{C})$ be a linear semisimple algebraic group defined over $\mathbb{Q}, G=\mathbf{G}(\mathbb{R})$ its real locus, a Lie group with finitely many components. Let $K \subset G$ be a maximal compact subgroup as usual, and $X=G / K$ the associated Riemannian symmetric space of noncompact type.

Let $\Gamma \subset \mathbf{G}(\mathbb{Q})$ be an arithmetic subgroup, i,e., a subgroup commensurable to $\mathbf{G}(\mathbb{Q}) \cap G L(n, \mathbb{Z})$. For simplicity, assume that $\Gamma$ is torsion free or even neat in the following. Then $\Gamma \backslash X$ is a locally symmetric space of finite volume.

Let $r$ be the $\mathbb{Q}$-rank of $\mathbf{G}$, which is defined to be the maximal dimension of $\mathbb{Q}$-split tori contained in $\mathbf{G}$. It is known that $\Gamma \backslash X$ is compact if and only if the $\mathbb{Q}$-rank $r$ of $\mathbf{G}$ is positive. In the following, we assume that $r>0$, and hence $\Gamma \backslash X$ is noncompact.

Let $\Delta_{\mathbb{Q}}(\mathbf{G})$ be the spherical Tits building of the algebraic group $\mathbf{G}$ defined over $\mathbb{Q}$, i.e., its simplexes correspond to $\mathbb{Q}$-parabolic subgroups. Then $\mathbf{G}(\mathbb{Q})$ and hence $\Gamma$ acts simplicially on $\Delta_{\mathbb{Q}}(\mathbf{G})$. Note that for any element $g \in \mathbf{G}(\mathbb{Q})$ and $x \in \Delta_{\mathbb{Q}}(\mathbf{G})$, if $g$ fixes $x$, then $g$ acts as the identity map on the unique simplex which contains $x$ as an interior point. Since there are only finitely many $\Gamma$-conjugacy classes of $\mathbb{Q}$-parabolic subgroups and $\Gamma$ acts, the quotient $\Gamma \backslash \Delta_{\mathbb{Q}}(\mathbf{G})$ is a finite simplicial complex. 
The basic theme of this and the next subsection is that the geometry at infinity and compactifications of $\Gamma \backslash X$ are closely related to $\Delta_{\mathbb{Q}}(\mathbf{G})$ and $\Gamma \backslash \Delta_{\mathbb{Q}}(\mathbf{G})$. For example, when $r=1, \Gamma \backslash \Delta_{\mathbb{Q}}(\mathbf{G})$ consists of finitely many points, which are in canonical one-to-one correspondence with the ends of $\Gamma \backslash X$.

\section{Borel-Serre compactification}

A well-known compactification of $\Gamma \backslash X$ is the Borel-Serre compactification in [BoS]. The compactification has also been discussed in many papers, for example, [GHM] [RS] [Ro2] [Gra1] [Gra2] [JM] [BJ1] [BJ2]. We will outline the modified approach in [JM] [BJ1] [BJ2].

It is constructed in the following three steps:

1. For every $\mathbb{Q}$-parabolic subgroup $\mathbf{P}$ of $\mathbf{G}$, choose a boundary component $e(\mathbf{P})$.

2. Attach all the boundary components $e(\mathbf{P})$ to $X$ at infinity to form a partial compactification ${ }^{1}$ $X \cup \coprod_{\mathbf{P}} e(\mathbf{P})$.

3. Show that the $\Gamma$-action on $X$ extends to a continuous action on $X \cup \coprod_{\mathbf{P}} e(\mathbf{P})$ with a compact quotient $\Gamma \backslash X \cup \coprod e(\mathbf{P})$.

In this general approach, the Langlands decomposition of $\mathbb{Q}$-parabolic subgroups and the induced horospherical decomposition of $X$ play a fundamental role.

For each $\mathbb{Q}$-parabolic subgroup $\mathbf{P}$, let $P=\mathbf{P}(\mathbb{R})$ be its real locus. Let $N_{P}$ be the unipotent radical of $P$. Let $\theta$ be the Cartan involution associated with the maximal compact subgroup $K$. Then there are two subgroups $A_{\mathbf{P}}$ and $M_{\mathbf{P}}$ of $P$ which are stable under $\theta$ and

$$
M_{\mathbf{P}}=\cap_{\chi \in X_{\mathbb{Q}}(\mathbf{G})}\{g \in G|| \chi(g) \mid=1\},
$$

where $X_{\mathbb{Q}}(\mathbf{G})$ is the set of characters of $\mathbf{G}$ defined over $\mathbb{Q}$, such that

$$
P=N_{P} A_{\mathbf{P}} M_{\mathbf{P}} \cong N_{P} \times A_{\mathbf{P}} \times M_{\mathbf{P}}
$$

The component $A_{\mathbf{P}}$ is diffeomorphic to its Lie algebra and called the $\mathbb{Q}$-split component. The product $A_{\mathbf{P}} M_{\mathbf{P}}$ is the Levi component of $\mathbf{P}$. The decomposition of $\mathbf{P}$ in Equation 2.11.1 is called the $\mathbb{Q}$-Langlands decomposition of $P$, since it depends on the $\mathbb{Q}$-structure of $\mathbf{P}$. In general, it is different from the $\left(\mathbb{R}\right.$-)Langlands decomposition of $\mathbf{P}$ recalled in Equation 2.6.1. In fact, $A_{\mathbf{P}} \subset A_{P}$ in general.

Define

$$
X_{\mathbf{P}}=M_{\mathbf{P}} / K \cap M_{\mathbf{P}},
$$

called the $\mathbb{Q}$-boundary symmetric space of $\mathbf{P}$. Then the $\mathbb{Q}$-Langlands decomposition of $P$ induces the $\mathbb{Q}$-horospherical decomposition

$$
X=N_{P} \times A_{\mathbf{P}} \times X_{\mathbf{P}} .
$$

Unlike the (R-)boundary symmetric space in Equation $2.6 .2, X_{\mathbf{P}}$ is in general the product of a symmetric space of noncompact type with a Euclidean space.

\footnotetext{
${ }^{1}$ By a partial compactification of $X$, we mean any space $Y$ which contains $X$ as an open dense subset. The reason is that $Y$ is obtained by compactifying $X$ in some directions or parts. Some other names for such spaces are bodifications and enlargements.
} 
Now the Borel-Serre compactification can be constructed as follows. For each $\mathbb{Q}$-parabolic subgroup $\mathbf{P}$, define its boundary component $e(\mathbf{P})$ by

$$
e(\mathbf{P})=N_{P} \times X_{\mathbf{P}}
$$

It is attached at the infinity of $X$ using the $\mathbb{Q}$-horospherical decomposition. In fact, the attachment is achieved when the $A_{\mathbf{P}}$-component goes to infinity through the positive chamber and stays further and further away from its walls.

Then the Borel-Serre partial compactification $\bar{X}^{B S}$ of $X$ is defined to be

$$
\bar{X}^{B S}=X \cup \coprod_{\mathbf{P}} N_{P} \times X_{\mathbf{P}}
$$

with a suitable topology. In fact, as mentioned above, the convergence of interior points to the boundary points in $X_{P}$ is described in terms of the $\mathbb{Q}$-horospherical decomposition of $X$ with respect to $\mathbf{P}$. For any two $\mathbb{Q}$-parabolic subgroups $\mathbf{P}$ and $\mathbf{Q}, e(\mathbf{P})$ is contained in the closure of $e(\mathbf{Q})$ if and only if $\mathbf{P} \subseteq \mathbf{Q}$; and the convergence sequences of points in $e(\mathbf{Q})$ to points is described in terms of a relative $\mathbb{Q}$-horospherical decomposition.

The $\Gamma$-action on $X$ extends to a continuous action on $\bar{X}^{B S}$. Since the boundary components $e(\mathbf{P})$ are sufficiently large, $\Gamma$ also acts properly on $\bar{X}^{B S}$. Using the reduction theory of arithmetic groups, it can be shown that the quotient $\Gamma \backslash \bar{X}^{B S}$ is a compact Hausdorff space, which is defined to be the Borel-Serre compactification of $\Gamma \backslash X$, also denoted by $\overline{\Gamma \backslash X}^{B S}$.

If $\Gamma$ is neat, then $\Gamma$ acts freely on $\bar{X}^{B S}$, and the quotient $\overline{\Gamma \backslash X}^{B S}$ is a compact real analytic manifold with corners.

When $\mathbf{G}=S L(2), \Gamma \backslash X$ is a Riemann surface with finitely many cusps, and $\overline{\Gamma \backslash X}^{B S}$ is obtained by adding one (horo)-circle to each cusp of $\Gamma \backslash X$. In this case, $\overline{\Gamma \backslash X}^{B S}$ is a real analytic manifold with boundary, adding one circle at each end of $\Gamma \backslash X$.

An important property of $\overline{\Gamma \backslash X}^{B S}$ is that when $\Gamma$ is neat, the inclusion $\Gamma \backslash X \rightarrow \overline{\Gamma \backslash X}^{B S}$ is a homotopy equivalence. In fact, $\overline{\Gamma \backslash X}^{B S}$ is a manifold with corners with the interior equal to $\Gamma \backslash X$. Since $\Gamma \backslash X$ is a $K(\Gamma, 1)$-space, $\overline{\Gamma \backslash X}^{B S}$ is a also $K(\Gamma, 1)$-space in this case. Since $\Gamma \backslash \bar{X}^{B S}$ is a compact real analytic manifold with corners and hence admits a finite triangulation, it follows that $\overline{\Gamma \backslash X} B S$ is a finite $K(\Gamma, 1)$-space.

The Borel-Serre compactification $\overline{\Gamma \backslash X}^{B S}$ is important in studying the cohomology groups of $\Gamma$. Its applications to the cohomology groups of $\Gamma$ have been discussed in many articles and surveys [Se2] [RS] [Br1] [Br3] [Ro2], and we will not discuss them except mentioning that the cohomological dimension of $\Gamma$ is equal to $\operatorname{dim} \Gamma \backslash X-r$, where $r$ is the $\mathbb{Q}$-rank of $\mathbf{G}$. In proving this result, the fact that the boundary $\partial \bar{X}^{B S}$ is a cell complex homotopy equivalent to the Tits building $\Delta_{\mathbb{Q}}(\mathbf{G})$ was used.

\section{Tits compactification}

In [JM], the general approach outlined above to compactify $\Gamma \backslash X$ was used to construct a partial compactification $\bar{X}^{T}$ whose boundary is exactly the Tits building $\Delta_{\mathbb{Q}}(\mathbf{G})$, and the quotient $\Gamma \backslash \bar{X}^{T}$ is a compactification whose boundary is the finite simplicial complex $\Gamma \backslash \Delta_{\mathbb{Q}}(\mathbf{G})$. Due to the relation to the Tits building $\Delta_{\mathbb{Q}}(\mathbf{G})$, this compactification of $\Gamma \backslash X$ was called the Tits compactification in $[\mathrm{JM}]$.

Briefly, the Tits compactification is constructed as follows. Let $\mathbf{G}$ be a linear semisimple algebraic group defined over $\mathbb{Q}$ as above. For each $\mathbb{Q}$-parabolic subgroup $\mathbf{P}$, let $\mathfrak{a}_{\mathbf{P}}$ be the Lie 
algebra of $A_{\mathbf{P}}$. The parabolic subgroup $\mathbf{P}$ determines a positive chamber $\mathfrak{a}_{\mathbf{P}}^{+}$. Let $\mathfrak{a}_{\mathbf{P}}^{+}(\infty)$ be the set of unit vectors in $\mathfrak{a}_{\mathbf{P}}^{+}$,

$$
\mathfrak{a}_{\mathbf{P}}^{+}(\infty)=\left\{H \in \mathfrak{a}_{\mathbf{P}}^{+} \mid\|H\|=1\right\},
$$

where $\|\cdot\|$ is the restriction of the Killing form to $\mathfrak{a}_{\mathbf{P}}$. Clearly $\mathfrak{a}_{\mathbf{P}}^{+}(\infty)$ is an open simplex. Define the boundary component $e(\mathbf{P})$ by

$$
e(\mathbf{P})=\mathfrak{a}_{\mathbf{P}}^{+}(\infty) \text {. }
$$

We also attach the boundary components $\mathfrak{a}_{\mathbf{P}}^{+}(\infty)$ to the infinity of $X$ through the $\mathbb{Q}$-horospherical decomposition associated with $\mathbf{P}$, and obtain the partial Tits compactification

$$
\bar{X}^{T}=X \cup \coprod_{\mathbf{P}} \mathfrak{a}_{\mathbf{P}}^{+}(\infty)
$$

It can be shown that $\Gamma$ acts continuously with a compact quotient $\Gamma \backslash \bar{X}^{T}$, which is also denoted by $\overline{\Gamma \backslash X}^{T}$.

\subsection{Geodesic compactification, Gromov compactification and large scale ge- ometry}

The Tits compactification $\overline{\Gamma \backslash X}^{T}$ is closely related to the structure of geodesics in $\Gamma \backslash X$ and the large scale geometry of $\Gamma \backslash X$. In fact, it is homeomorphic to the geodesic compactification $\Gamma \backslash X \cup \Gamma \backslash X(\infty)$ and the Gromov compactification $\overline{\Gamma \backslash X}^{G}$, and its boundary $\Gamma \backslash \Delta_{\mathbb{Q}}(\mathbf{G})$ is also the base of the cone of the tangent space at infinity $T_{\infty} \Gamma \backslash X$. Furthermore, the cone over $\Gamma \backslash \Delta_{\mathbb{Q}}(\mathbf{G})$ is within a finite Gromov-Hausdorff distance of $\Gamma \backslash X$, and this result is closely related to a conjecture of Siegel.

\section{Geodesic compactification $\Gamma \backslash X \cup \Gamma \backslash X(\infty)$}

In the rest of this subsection, $X$ denotes the symmetric space $G / K$ of noncompact type, and a general Riemannian manifold is denoted by $M$. As pointed out earlier, $X$ is simply connected and nonpositively curved, and hence the set of equivalence classes of geodesics defines the sphere at infinity $X(\infty)$, which is the boundary of the geodesic compactification $X \cup X(\infty)$.

On the other hand, since $\Gamma \backslash X$ is noncompact, there are geodesics which are bounded, or unbounded but do not go to infinity. It is necessary to choose a suitable class of geodesics. In [JM], EDM geodesics were used to define the geodesic compactification $\Gamma \backslash X \cup \Gamma \backslash X(\infty)$.

Specifically, a (unit speed, directed) geodesic $\gamma(t)$ in $\Gamma \backslash X$ is called eventually distance minimizing (EDM) if for all $t_{1}, t_{2} \gg 0$,

$$
d\left(\gamma\left(t_{1}\right), \gamma\left(t_{2}\right)\right)=\left|t_{1}-t_{2}\right|
$$

Clearly, every EDM geodesic $\gamma(t)$ goes to infinity of $\Gamma \backslash X$, i.e., leaves every compact subset of $\Gamma \backslash X$, as $t \rightarrow+\infty$. Two EDM geodesics $\gamma_{1}, \gamma_{2}$ are called equivalent if

$$
\limsup _{t \rightarrow+\infty} d\left(\gamma_{1}(t), \gamma_{2}(t)\right)<+\infty .
$$

Let $\Gamma \backslash X(\infty)$ be the set of equivalence classes of EDM geodesics in $\Gamma \backslash X$.

EDM geodesics were studied in [Ha1] [Ha2] [Le2], and were completely classified in [JM]. In particular, $\Gamma \backslash X(\infty)$ was canonically identified with $\Gamma \backslash \Delta_{\mathbb{Q}}(\mathbf{G})$.

In $[\mathrm{JM}]$, a general procedure was given to compactify a complete (not necessarily simply connected) Riemannian manifold which satisfies certain conditions by adding the set of equivalence classes of EDM geodesics. It was then shown that these conditions were satisfied by $\Gamma \backslash X$, and hence 
the geodesic compactification $\Gamma \backslash X \cup \Gamma \backslash X(\infty)$ exists. Since $\Gamma \backslash X(\infty)=\Gamma \backslash \Delta_{\mathbb{Q}}(\mathbf{G})$, the geodesic compactification is the same as the Tits compactification $\overline{\Gamma \backslash X}^{T}$.

\section{Gromov compactification}

In [BGS], Gromov introduced a compactification of any complete Riemannian manifold $M$ using the distance functions. Let $C^{0}(M)$ be the space of continuous functions on $M$, and $\tilde{C}^{0}(M)$ be the quotient of $C^{0}(M)$ by the space of constant functions. Define a map

$$
i_{G}: M \rightarrow \tilde{C}^{0}(M), \quad x \mapsto[d(x, \cdot)]
$$

where $d(x, \cdot)$ is the distance from $x$, and $[d(x, \cdot)]$ its image in $\tilde{C}^{0}(M)$. It can be shown that the map $i_{G}$ is an embedding, and the closure of $i_{G}(X)$ in $\tilde{C}^{0}(M)$ is compact and called the Gromov compactification and denoted by $\bar{M}^{G}$.

When $M$ is a symmetric space $X$ of noncompact type, it was shown in [BGS] that $\bar{X}^{G} \cong$ $X \cup X(\infty)$. When $M=\Gamma \backslash X$, it was shown in [JM] that $\overline{\Gamma \backslash X}^{G} \cong \overline{\Gamma \backslash X}^{T}$ and hence

$$
\overline{\Gamma \backslash X}^{G}=\Gamma \backslash X \cup \Gamma \backslash X(\infty) .
$$

\section{Tangent cone at infinity}

For any Riemannian manifold $(M, g)$, and a point $p \in M$, the tangent space $T_{p} M$ can be obtained as the Gromov-Hausdorff limit of $(M, \varepsilon g)$ with the fixed point $p$ as $\varepsilon \rightarrow 0$. On the other hand, if the Gromov-Hausdorff limit of $\left(M, \frac{1}{\varepsilon} g\right)$ exists as $\varepsilon \rightarrow 0$, it called the tangent space of $M$ at infinity, denoted by $T_{\infty} M$ (For general manifolds, we need to take ultralimits in order to get existence of the tangent cone. See $\S 4$ below about how the tangent cone of symmetric spaces leads to $\mathbb{R}$-trees and $\mathbb{R}$-Euclidean buildings). Clearly, it is independent of the choice of the point $p$ and only depends on the quasi-isometry class of the metric $g$, and hence describes the geometry at infinity. It was shown in $[\mathrm{JM}]$ that $T_{\infty} \Gamma \backslash X$ exists and is equal to a cone $C\left(\Gamma \backslash \Delta_{\mathbb{Q}}(\mathbf{G})\right)$, a metric cone with a homothety section given by $\Gamma \backslash \Delta_{\mathbb{Q}}(\mathbf{G})$.

In [J3], it was shown that the Gromov-Hausdorff distance between $\Gamma \backslash X$ and $C\left(\Gamma \backslash \Delta_{\mathbb{Q}}(\mathbf{G})\right)$ is finite. This is closely related to a conjecture of Siegel which compares metrics of $X$ and $\Gamma \backslash X$ on Siegel sets. See [J3] for details.

\section{Euclidean buildings}

In this section, we discuss applications of Euclidean buildings to super-rigidity of discrete subgroups of co-finite volume in the isometry groups of the quaternionic hyperbolic spaces and the Cayley hyperbolic plane, and integral Novikov conjectures for S-arithmetic groups.

First we discuss Euclidean reflection groups and Euclidean buildings, and construct the BruhatTits buildings of semisimple simply connected algebraic groups defined over a field with discrete valuation. The key point is that instead of parabolic subgroups, we use parahoric subgroups to parametrize simplexes of the buildings. Then we discuss choices of $B N$-pairs and buildings for non-simply connected algebraic groups and a concrete realization of the Bruhat-Tits building of the group $S L(n)$.

Once the Euclidean buildings are defined, we study their applications. The first concerns the proof by Garland of a conjecture of Serre on vanishing of certain cohomology groups of uniform discrete subgroups of $p$-adic Lie groups. 
The second is the proof by Gromov and Schoen of the non-archimedean super-rigidity of cofinite discrete subgroups of $S p(1, n)$ and $F_{4(-20)}$. To motivate this, we recall the Margulis super-rigidity in the higher rank case. Since the latter part of the arguments in [GS] follows the proof in [Co], we briefly outline the proof in [Co] for archimedean super-rigidity of the cofinite discrete subgroups of $S p(1, n)$ and $F_{4(-20)}$, and comment on their differences which lead to the boundedness of the image of the representation.

To obtain applications to $S$-arithmetic subgroups, we need to compactify Euclidean buildings by spherical Tits buildings. We conclude this section by an application to the integral Novikov conjectures for $S$-arithmetic subgroups.

\subsection{Definitions and basic properties}

Let $V$ be a Euclidean space. An affine reflection group $W$ on $V$ is a group of affine isometries generated by reflections with respect to affine hyperplanes such that the set $\mathcal{H}$ of affine hyperplanes fixed by reflections in $W$ is locally finite. Clearly, a finite reflection group is an affine reflection group.

The linear parts of the affine transformations in $W$ define a finite (linear) reflection group $\bar{W}$. $W$ is called essential if $\bar{W}$ is essential.

The hyperplanes in $\mathcal{H}$ divide $V$ into chambers, and $W$ acts simply transitively on the set of chambers. Let $C$ be a chamber. Then $W$ is generated by the reflections of the walls of $C$.

Proposition 3.1.1 Assume that $W$ is essential and irreducible. Then

1. either $W$ is finite and has a fixed point, and hence becomes a finite reflection group when the point becomes the origin. In this case, $C$ is a simplicial cone;

2. or $W$ is infinite and $C$ is a simplex.

See [Br1, Chap VI., §1] for a proof of this proposition. An essential infinite affine reflection group is called a Euclidean reflection group. The chambers and their faces form a simplicial complex, which is called a Euclidean Coxeter complex. A typical example of a Euclidean reflection group is the affine Weyl group of a simple Lie algebra over $\mathbb{C}$.

Definition 3.1.2 A chamber complex $\Delta$ is called a Euclidean building if it satisfies all the conditions in Definition 2.1.3 except that all the apartments are Euclidean Coxeter complexes.

Since the underlying space of each Euclidean Coxeter complex is a Euclidean space, it has a metric. Fix a Euclidean metric on every apartment such that all apartments are isometric. Then these metrics can be glued into a metric on $\Delta$ which is a geodesic space, i.e., the distance between any two points is realized by a geodesic connecting them [Br1, Chap. VI, §3] [BH].

Proposition 3.1.3 Any Euclidean building $\Delta$ as a metric space is a CAT(0)-space, and hence has nonpositive curvature and is contractible. In particular, it is simply connected.

Recall that a CAT(0)-space $M$ is a length space such that every triangle in $M$ is thinner than a corresponding triangle in $\mathbb{R}^{2}$ of the same side lengths $[\mathrm{BH}]$. This proposition has important application to understanding compact subgroups of semisimple $p$-adic groups. 


\subsection{Semisimple $p$-adic groups and Euclidean Buildings}

Next we consider some examples of Euclidean buildings constructed from linear semisimple algebraic groups defined over local fields.

Let $\mathbf{G}$ be a linear connected semisimple algebraic group defined over a non-archimedean local field $F$, i.e., a field with a complete discrete valuation. Assume that $F$ is locally compact, which is equivalent to that the residue field $f$ is finite. For example, we can take $F=\mathbb{Q}_{p}$, where $p$ is a finite prime, and the residue field is $f=\mathbb{F}_{p}$. We also assume that $\mathbf{G}$ is absolutely almost simple, i.e., over any field extension of $F, \mathbf{G}$ has no proper normal subgroup of strictly positive dimension.

In the first part, we assume that $\mathbf{G}$ is simply connected, i.e., there is no nontrivial isogeny $\mathbf{G}^{\prime} \rightarrow \mathbf{G}$ over $K$. Examples include $\mathbf{G}=S L(n), S p(n)$. In the second part, we consider the nonsimply connected case. Then we conclude this subsection with the example of the Bruhat-Tits building of $S L(n)$.

Simply connected semisimple algebraic groups

In $\S 2.2$, for any linear semisimple algebraic group $\mathbf{G}$ defined over any field $F$, we can associate a spherical Tits building $\Delta(\mathbf{G})$, whose simplexes correspond to parabolic subgroups of $\mathbf{G}$ which are defined over $F$. When the parabolic subgroups are replaced by parahoric subgroups of $\mathbf{G}(F)$, we obtain a Euclidean building $\Delta^{B T}(\mathbf{G})$, called the Bruhat-Tits building of $\mathbf{G}$.

As recalled in $\S 2.2$, parabolic subgroups $\mathbf{P}$ of $\mathbf{G}$ can be defined simply as subgroups such that $\mathbf{G} / \mathbf{P}$ is a projective variety. On the other hand, there is no such simple characterization of parahoric subgroups of $\mathbf{G}(F)$ for general reductive group $\mathbf{G}$ (see [BT2, Definition 5.2.6] and also [BT1, p.31]).

Fortunately, under our assumption that $\mathbf{G}$ is simply connected and absolutely almost simple, there is a direct definition of parahoric subgroups. A minimal parahoric subgroup of $\mathbf{G}(F)$ is called an Iwahori subgroup and is equal to the normalizer of any maximal pro- $p$-subgroup of $\mathbf{G}(F)$, where $p$ is the characteristic of the residue field $f$, and a pro- $p$-subgroup is the projective limit of a sequence of $p$-subgroups. Such minimal parahoric subgroups are conjugate under $\mathbf{G}(F)$. Then any proper subgroup of $\mathbf{G}(F)$ containing an Iwahori subgroup is called a parahoric subgroup of $\mathbf{G}(F)$.

When $\mathbf{G}=S L(n)$, examples of Iwahori and parahoric subgroups are described explicitly in [Br1, Chap. V, §8] (see below).

The relations between the parahoric subgroups are similar to those of parabolic subgroups.

Proposition 3.2.1 Under the above assumptions on $\mathbf{G}$, all minimal parahoric subgroups of $\mathbf{G}(F)$ are conjugate. Fix any minimal parahoric subgroup B. Let $r$ be the rank of $\mathbf{G}$ over $F$. Then there are exactly $r+1$ maximal parahoric subgroups $P_{1}, \cdots, P_{r+1}$ which contain $B$, and $P_{i_{1}} \cap \cdots \cap P_{i_{j}}$ are exactly all the parahoric subgroups which contain $B$ when $\left\{i_{1}, \cdots, i_{k}\right\}$ runs over non-empty subsets of $\{1, \cdots, r+1\}$. In particular, any parahoric subgroup is conjugate to such an intersection.

Parahoric subgroups are basically related to bounded subgroups and compact open subgroups of $\mathbf{G}(F)$.

Definition 3.2.2 A subgroup $K \subset \mathbf{G}(F)$ is called bounded if it satisfies one of the following equivalent conditions:

1. There exists a faithful finite dimensional rational representation $\rho: \mathbf{G}(F) \rightarrow G L(n, F)$ defined over $F$ such that the coordinates of the elements $\rho(g), g \in K$, are uniformly bounded with respect to the natural topology of $F$.

2. For any finite dimensional rational representation $\rho: \mathbf{G}(F) \rightarrow G L(n, F)$, the coordinates of the elements $\rho(g), g \in K$, are uniformly bounded with respect to the natural topology of $F$. 
3. For any finite dimensional rational representation $\rho: \mathbf{G}(F) \rightarrow G L(n, F)$, there exists a lattice $\Lambda$ in $F^{n}$ such that $\rho(g) \Lambda=\Lambda, g \in K$.

By (3), any bounded subgroup is contained in a compact open subgroup of $\mathbf{G}(F)$. When $\mathbf{G}$ is simply connected, any maximal open compact subgroup of $\mathbf{G}(F)$ is a maximal parahoric subgroup, and the converse is also true.

Remark 3.2.3 To emphasize the similarities between the parabolic subgroups and parahoric subgroups, we point out that when $\mathbf{G}$ is split over $F$, then the minimal parahoric subgroups, i.e., the Iwahori subgroups, are lifted from the Borel subgroups of the reduction modulo the prime ideal of some integral model of $\mathbf{G}$ (see [IM] $[\mathrm{Iw}]$ and the example of $\mathbf{G}=S L(n)$ in [Br1, p. 130]). In this split case, the minimal parabolic subgroups of $\mathbf{G}$ are given by the Borel subgroups, i.e., the maximal connected solvable subgroups.

The Bruhat-Tits building $\Delta^{B T}(\mathbf{G})$ is defined to be the simplicial complex whose simplexes correspond to parahoric subgroups such that

1. The maximal parahoric subgroups correspond to vertexes.

2. The inclusion relation is the opposite of the inclusion relation of the parahoric subgroups. In particular, for any parahoric subgroup $P$, the vertexes of its simplex correspond to the maximal parahoric subgroups which contain $P$.

The apartments in $\Delta^{B T}(\mathbf{G})$ are also similarly described as in the case of the spherical Tits building $\Delta(\mathbf{G})$. Let $\mathcal{O}_{F}$ be the ring of valuation. Then for any maximal $F$-split torus $\mathbf{T}$ of $\mathbf{G}$, the set of parahoric subgroups which contain $\mathbf{T}\left(\mathcal{O}_{F}\right)$ form an apartment in $\Delta^{B T}(\mathbf{G})$ and is isomorphic to a Euclidean Coxeter complex.

As pointed out in $\S 2.3$, buildings can also be described in terms of $B N$-pairs. In this case, $B$ is a minimal parahoric subgroup, and $\mathbf{T}$ is a maximal $F$-split torus such that $\mathbf{T}(F)$ is contained in $B$. Let $N$ be the normalizer of $\mathbf{T}(F)$ in $\mathbf{G}(F)$. Then $B$ and $N$ form a $B N$-pair, i.e., they satisfy the axioms in Definition 2.3.1. The intersection $B \cap N$ is a maximal compact subgroup of the centralizer of $\mathbf{T}(F)$ in $\mathbf{G}(F)$, and $W=N /(B \cap N)$ is a Euclidean Coxeter group whose Coxeter complex is isomorphic to the apartments in $\Delta^{B T}(\mathbf{G})$. In fact, $W$ is the extension of a finite Coxeter group by a free abelian group of rank equal to the $F$-rank of $\mathbf{G}$. Such a Weyl group is called an affine Weyl group.

Since $\mathbf{G}(F)$ acts on the set of parahoric subgroups by conjugation, it acts simplically on $\Delta^{B T}(\mathbf{G})$ such that the stabilizers of the vertexes are the maximal parahoric subgroups.

Remark 3.2.4 When $\mathbf{G}$ is absolutely almost simple and simple connected over $F=\mathbb{Q}_{p}$ or more general local fields, the existence of $B N$-pairs with affine Weyl groups in $\mathbf{G}(F)$ is proved in [Hij]. Such $B N$-pairs with affine Weyl groups are unique up to conjugation. On the other hand, for a general reductive linear group $\mathbf{G}$ defined over a local field, the Bruhat-Tits buildings are constructed first using valued root data, and then parahoric subgroups are defined in terms of stabilizers of facets in Bruhat-Tits buildings, different from the procedure described above.

Remark 3.2.5 The basic idea in the approach using valued root data is to start with the structure of an apartment and to use the group action to obtain other apartments and hence the buildings. Roughly, let $A$ be an apartment. Then the Bruhat-Tits building $\Delta^{B T}(\mathbf{G})$ is defined as a quotient of $G \times A$. Such an idea of passing from an apartment to the whole buildings was also used in 
constructing compactifications of Bruhat-Tits buildings in [Lan2]. It is also needed to construct non-discrete $\mathbb{R}$-Euclidean buildings discussed in $\S 4$ below.

A related idea of passing from a totally geodesic maximal flat subspace of a symmetric space $X$ of noncompact type to the symmetric space has been used to understand compactifications of symmetric spaces in [Os] and [GJT, Chap. III].

Remark 3.2.6 For a semisimple algebraic group $\mathbf{G}$ as in the above Remark, besides the $B N$-pair which gives the Bruhat-Tits building, there is also a $B N$-pair in $\S 2.3$ which leads to the spherical Tits building corresponding to the parabolic subgroups. The first $B N$-pair depends on the structure of the local fields, while the second depends on the structure of the algebraic group $\mathbf{G}$.

Non-simply connected semisimple groups

Let $\mathbf{G}$ be a connected, non-simply connected linear semisimple algebraic group defined over a non-archimedean local field $F$. Let $\tilde{\mathbf{G}}$ be its universal covering. Then $\tilde{\mathbf{G}}$ is a simply connected semisimple linear algebraic group defined over $F$, and hence its Bruhat-Tits building of $\Delta^{B T}(\tilde{\mathbf{G}})$ is defined as above. Define the Bruhat-Tits building $\Delta^{B T}(\mathbf{G})$ to be equal to $\Delta^{B T}(\tilde{\mathbf{G}})$. Let $\pi: \tilde{\mathbf{G}} \rightarrow \mathbf{G}$ be the canonical central isogeny. The group $\tilde{\mathbf{G}}(F)$ acts on the set of its parahoric subgroups via conjugation. Since the center of $\tilde{\mathbf{G}}(F)$ clearly acts trivially, this gives an action of $\mathbf{G}(F)$ on the set of parahoric subgroups of $\tilde{\mathbf{G}}(F)$ and hence on the building $\Delta^{B T}(\tilde{\mathbf{G}})$ (see $[\operatorname{BoS1}, \S 4.8]$ ).

Exmaple: Bruhat-Tits building of $S L(n)$.

For the sake of explicitness, we describe the $B N$-pair in the case $\mathbf{G}=S L(n)$ over $\mathbb{Q}_{p}$, where $p$ is a finite prime [Br1, Chap. $\mathrm{V}, \S 8]$. Let $S L\left(n, \mathbb{F}_{p}\right) \rightarrow S L\left(n, \mathbb{F}_{p}\right)$ be the natural projection. Take $B$ to be the inverse image of the upper triangular subgroup, and $N$ to be the monomial subgroup of $S L\left(n, \mathbb{Q}_{p}\right)$, i.e., the subgroup consisting of all matrices with exactly one non-zero element in every row and every column.

The simplexes and apartments in $\Delta^{B T}(S L(n))$ can also be described explicitly in terms of lattices (see [Ro1, pp. 31-32] [Br1, pp. 132-137]). Briefly, two lattices $\Lambda$ and $\Lambda^{\prime}$ in $F^{n}$ are equivalent if there exists an element $\lambda \in F^{\times}$such that $\Lambda=\lambda \Lambda^{\prime}$. The stabilizers of equivalent lattices are obviously the same, and they are the maximal parahoric subgroups. Hence, the equivalence classes of lattices correspond to vertexes of $\Delta^{B T}(S L(n))$. Two equivalence classes $[\Lambda]$ and $\left[\Lambda^{\prime}\right]$ form the vertexes of a simplex of dimension 1 if and only if there exist representatives $\Lambda$ and $\Lambda^{\prime}$ such that

$$
\pi \Lambda \supset \Lambda^{\prime} \supset \Lambda,
$$

where $\pi$ is a uniformizer of the valuation ring of integer of the local field $F$. The vertexes $\left[\Lambda_{1}\right], \cdots,\left[\Lambda_{m}\right]$ form the vertexes of a $m$-simplex if there are representatives $\Lambda_{1}, \cdots, \Lambda_{m}$ such that

$$
\Lambda_{1} \supset \cdots \supset \Lambda_{m} \supset \pi \Lambda_{1} \text {. }
$$

If a lattice $\Lambda$ has a basis $v_{1}, \cdots, v_{n}$, denote the equivalence class of $\Lambda$ by $\left[v_{1}, \cdots, v_{n}\right]$. Let $e_{1}, \cdots, e_{n}$ be a basis of $F^{n}$. Then the vertexes $\left[e_{1}, \cdots, e_{i}, \pi e_{i+1}, \cdots, \pi e_{n}\right], i=1, \cdots, n$, form the vertexes of a chamber in $\Delta^{B T}(S L(n))$, a fundamental domain of the Euclidean Coxeter group acting on the Euclidean Coxeter complex.

The equivalence classes $\left[\pi^{i_{1}} e_{1}, \cdots, \pi^{i_{n}} e_{n}\right]$ form the vertexes of an apartment containing the above chamber, and any apartments are obtained from this apartment under the action of $S L(n, F)$.

Remark 3.2.7 See [BT3] [BT4] for concrete realization of the building in terms of norms for classical groups and [GY] for exceptional groups. See also [AbN] [Yu1] for other concrete realizations. See also [Yu2] for a survey of the Bruhat-Tits theory. 
Remark 3.2.8 In this case, there are $n+1 S L(n, F)$-conjugacy classes of maximal parahoric subgroups of $S L(n, F)$, which are also maximal compact subgroups of $S L(n, F)$. On the other hand, it is well-known that there is only one $S L(n, \mathbb{R})$-conjugacy class of maximal compact subgroups in $S L(n, \mathbb{R})$. To help explain this difference, we note that if $K \subset S L(n, F)$ is a maximal compact subgroup, then for any $g \in G L(n, F)$, the conjugate $g K^{-1}$ is also a maximal subgroup in $S L(n, F)$. But this conjugation can not always be achieved by elements in $S L(n, F)$. (This problem will not arise for $S L(n, \mathbb{R})$ since we can always assume $\operatorname{det} g>0$ and use $(\operatorname{det} g)^{-1 / n} g \in S L(n, \mathbb{R})$ to get the same conjugated subgroup). In fact, $G L(n, F)$ acts transitively on the set of lattices in $F^{n}$, and hence all the maximal compact subgroups of $S L(n, F)$ are conjugate under $G L(n, F)$.

When the $F$-rank of $\mathbf{G}$ is equal to $1, \Delta^{B T}(\mathbf{G})$ is a tree. For a thorough discussion of trees and their applications to combinatorial group theory, see [Se1].

\section{$3.3 \quad p$-adic curvature and vanishing of cohomology}

In [Kaz], Kazhdan introduced the important notion of Property (T) for a locally compact and second countable group $G$. Briefly, a group $G$ has the Property (T) if every unitary representation of $G$ which almost has invariant vectors does have nontrivial invariant vectors, i.e., the trivial representation is isolated in the unitary dual of $G$.

The Property $(\mathrm{T})$ has been very useful for many different problems, in particular for problems around rigidity of discrete subgroups of Lie groups. See [Zi, Chap. 7] for the precise definition and applications.

In [Wa], Wang showed that if $G$ has property (T) and $\pi$ is a unitary representation of $G$, then $H^{1}(G, \pi)=0$.

In [Kaz], Kazhdan proved that connected semisimple Lie groups with finite center and no simple factor of rank 1 have Property (T). Furthermore, any lattice subgroups $\Gamma$, i.e., discrete subgroups of finite covolume, of such Lie groups have Property $(\mathrm{T})$ as well. In particular, for such lattice subgroups, $H^{1}(\Gamma, \pi)=0$ for any unitary representation $\pi$ of $\Gamma$. This vanishing of $H^{1}(\Gamma, \pi)$ is closely related to the (infinitesimal) rigidity of $\Gamma$, or rather the associated locally symmetric space $\Gamma \backslash X$, where $X=G / K, K$ being a maximal compact subgroup of $G$ (see [Mos, p.5] for a history of results leading to Theorem 2.8.1 in $\S 2.8$, and results in [We1] [We2] [We3] [CV] [Mat1] [Mat2] [Ra1] [Ra2] [JoM] which are related to the first cohomology of $\Gamma$ ).

Let $k$ be either a number field or a function field of an algebraic curve over a finite field, and $k_{\mathfrak{p}}$ the completion at a finite place. Then $k_{\mathfrak{p}}$ is a local field.

Let $\mathbf{G}$ be a simple linear algebraic group defined over $k_{\mathfrak{p}}$, and $\Gamma \subset \mathbf{G}\left(k_{\mathfrak{p}}\right)$ a torsion free discrete and cocompact subgroup.

Remark 3.3.1 It should be pointed out that when the characteristic of $k_{\mathfrak{p}}$ is equal to zero, then any co-finite discrete subgroup $\Gamma$ of $\mathbf{G}\left(k_{\mathfrak{p}}\right)$ is uniform. In fact, if not, it will contain a nontrivial unipotent element, which will generate a non-discrete subgroup. The assumption on the characteristic of $k_{\mathfrak{p}}$ is necessary.

Assume that the $k_{\mathfrak{p}}$-rank $r$ of $\mathbf{G}$ is at least 2, then it is also known that $\Gamma$ has Property (T) (see [Mar, Chap. III, Theorem 5.3] [HV]); in particular,

$$
H^{1}(\Gamma, \pi)=0
$$

for any unitary representation $\pi$ of $G$. 
Motivated by this and his computations about signs of the Euler characteristic of $\Gamma$ in $[\mathrm{Se} 2]$, Serre conjectured that for any $i, 0<i<r$,

$$
H^{i}(\Gamma, \mathbb{R})=0
$$

When the rank $r=2$, this is reduced to Equation 3.3.1.

In [Garl], Garland proved this conjecture by using the Bruhat-Tits building $\Delta^{B T}$. (In [Garl], the cardinality of the residue field was assumed to be sufficiently large. This additional assumption was removed by Casselman in [Cas]). We will very briefly outline the idea of the proof.

Recall that for any group $\Gamma$ (with discrete topology), its $K(\Gamma, 1)$-space is defined to be a connected CW-space such that

$$
\pi_{1}(K(\Gamma, 1))=\Gamma, \quad \pi_{i}(K(\Gamma, 1))=\{1\}, \quad i \geq 2 .
$$

Then it is known that

$$
H^{i}(\Gamma, \mathbb{R})=H^{i}(K(\Gamma, 1), \mathbb{R})
$$

Since it is often difficult to compute $H^{i}(\Gamma, \mathbb{R})$ directly from the definition, it is crucial to find a good model of $K(\Gamma, 1)$.

If $\Gamma$ acts freely on some contractible space $Y$, then $\Gamma \backslash Y$ is a $K(\Gamma, 1)$-space.

Assume that $\Gamma$ is a torsion free discrete subgroup of a connected semisimple Lie group $G$. Let $K$ be a maximal compact subgroup of $G$, and $X=G / K$ the associated symmetric space of noncompact type, which is diffeomorphic to a Euclidean space and hence contractible. Then $G$ acts on $X$ by the left multiplication, and the stabilizer in $G$ of every point in $X$ is a compact subgroup of $G$. This implies that $\Gamma \backslash X$ is a $K(\Gamma, 1)$-space.

In the case under consideration, $\mathbf{G}\left(k_{\mathfrak{p}}\right)$ is a non-archimedean Lie group, and there is more than one conjugacy class of maximal compact subgroups $K$; and for each of them, the quotient $\mathbf{G}\left(k_{\mathfrak{p}}\right) / K$ is certainly not contractible. On the other hand, the Bruhat-Tits building $\Delta^{B T}(\mathbf{G})$ is a contractible simplicial complex, and $\mathbf{G}\left(k_{\mathfrak{p}}\right)$ acts simplicially on it such that the stabilizer in $\mathbf{G}\left(k_{\mathfrak{p}}\right)$ of every point is a compact subgroup. By assumption, $\Gamma$ is a disctere, cocompact subgroup of $\mathbf{G}\left(k_{\mathfrak{p}}\right)$. This implies that $\Gamma$ acts freely on $\Delta^{B T}(\mathbf{G})$ if $\Gamma$ is torsion free and discrete, and hence $\Gamma \backslash \Delta^{B T}(\mathbf{G})$ is a $K(\Gamma, 1)$ space. Furthermore, since the quotient of $\Delta^{B T}(\mathbf{G})$ by $\mathbf{G}\left(k_{\mathfrak{p}}\right)$ consists of a closed chamber, it follows that $\Gamma \backslash \Delta^{B T}(\mathbf{G})$ is a finite $\mathrm{CW}$-complex, which gives a so-called finite $K(\Gamma, 1)$-space.

So the conjecture of Serre is reduced to proving vanishing of $H^{i}\left(\Gamma \backslash \Delta^{B T}(\mathbf{G}), \mathbb{R}\right), 0<i<r$. In [Garl], Garland introduced a scalar product on the finite dimensional cochain complex, and the associated Laplace operator and hence the harmonic cocycles. The analogue of the Hodge decomposition shows that $H^{i}\left(\Gamma \backslash \Delta^{B T}(\mathbf{G}), \mathbb{R}\right)$ is isomorphic to the space of harmonic cocycles. The vanishing of these harmonic cocycles depends on lower bounds of eigenvalues of certain quadratic forms. This approach was suggested by the modification of the Bochner identity by Matsushima in [Mat1]. Since a corresponding quadratic form in [Mat1] depends on the curvature operator of the symmetric space, Garland called his operator $p$-adic curvature.

\subsection{Super-rigidity and harmonic maps into Euclidean buildings}

There have been several important generalizations of the Mostow strong rigidity. One is the Margulis super-rigidity [Mar], and another is for more general, not necessarily locally symmetric spaces by the approach of using harmonic maps and Bochner type formulas, which was initially suggested by S.T.Yau [Yau1, p. 37] to study both rigidity of discrete subgroups and complex structures of certain manifolds.

In fact, the Mostow rigidity was used by Yau to prove his rigidity of complex structures on $\mathbb{C} P^{2}$ as a corollary of his celebrated solution of Calabi's conjecture [Yau4, Theorem 6]. 
Theorem 3.4.1 Let $N$ be a compact quotient of the unit ball in $\mathbb{C}^{2}$ by a (uniform) lattice in $S U(1,2)$. Then any complex surface homotopy equivalent to $N$ preserving the canonical orientations induced from the complex structures is biholomorphic to $N$.

In the proof of this theorem, the Mostow strong rigidity was used. This is the first instance where the Mostow rigidity for locally symmetric spaces is combined with the rigidity of complex structures of Kähler manifolds (see also [Yau4, Remark after Theorem 5] on rigidity of the complex structure of $\left.\mathbb{C} P^{n}\right)$.

Motivated by these results, Yau suggested the idea of using harmonic maps to study both the rigidity of discrete subgroups and the rigidity of complex structures of Kähler manifolds with negative curvature [Yau1, p. 37] [Yau3] [JY3]. In [Siu2] [Siu1], Siu carried out this approach for the rigidity of the complex structures with a slightly stronger condition on negativity of the curvature.

The Siu-Yau method of using harmonic maps is very elegant and has also turned out to be extremely powerful for both Mostow type rigidity of lattices of Lie groups and the rigidity of geometric structures (see [JY1] [JY3] [MSY] [Mok1] [Mok2] and the references there). In fact, it can be used to reprove the Mostow strong rigidity and the Margulis super-rigidity in many cases ([MSY] [JY1] [JZ]). More importantly, it can be used to prove new cases of super-rigidity, i.e., the superrigidity of lattices acting on the quaternionic hyperbolic spaces and the Cayley hyperbolic plane, which can not be proved by other methods. More specifically, Corlette proved the archimedean super-rigidity of such lattices in [Co], and Gromov and Schoen developed a theory of harmonic maps to the Bruhat-Tits buildings and used it to prove the non-archimedean super-rigidity of these lattices in [GS].

Remark 3.4.2 The generalization of the Mostow strong rigidity to $\mathfrak{p}$-adic Lie groups and lattices is given in [Pr2]. Another generalization of rigidity for lattices in $P G L(d, k)$, where $k$ is a local non-Archimedean field, is given in [Ber1]. A generalization to graphs, in particular to quotients of Bruhat-Tits trees, of the entropy rigidity of rank 1 locally symmetric spaces in [BCG] is given in [Lim].

Remark 3.4.3 Some other versions of rigidity of lattices in $P U(1,2)$, for example, the superrigidity of lattices satisfying some cohomological conditions and integrality of lattice subgroups, are given in [Klig1] and [Ye1] [Ye2]. More precisely, let $\mathbf{G} \subset G L(n)$ be an algebraic group defined over $\mathbb{Q}$. A subgroup $\Gamma \subset \mathbf{G}(\mathbb{R})$ is called integral if it contains a subgroup $\Gamma^{\prime}$ of finite index such that $\Gamma^{\prime} \subset \mathbf{G}(\mathbb{Q}) \cap G L(n, \mathbb{Z})$. If $\Gamma^{\prime}$ is also of finite index in $\mathbf{G}(\mathbb{Q}) \cap G L(n, \mathbb{Z})$, then $\Gamma$ is an arithmetic subgroup. The symmetric space of noncompact type $X=P U(1,2) / U(2)$ can be identified with the complex unit ball $B_{\mathbb{C}}^{2}$ in $\mathbb{C}^{2}$. One result in [Ye1] says that if a ball quotient $\Gamma \backslash B_{\mathbb{C}}^{2}$ has the first Betti number equal to 1 and the Picard number equal to 1 , then $\Gamma$ is integral. Under other conditions, the results in [Ye2] and [Klig1] show that $\Gamma$ is arithmetic. In the proofs of these results, Bruhat-Tits buildings played an important role. See also [PYe] for applications of Bruhat-Tits buildings in classifying fake projective planes, where a fake projective plane is a smooth compact complex surface which is not the complex projective plane but has the same first and second Betti numbers as the complex projective plane.

Remark 3.4.4 The idea of using harmonic maps is very fruitful. Besides the applications in [Co] and [GS], it has also been used by Sampson [Sa], Simpson [Sim], Carlson-Toledo [CT] and others. As well-known, the Mostow strong rigidity fails for hyperbolic Riemann surfaces. On the other hand, the theory of harmonic maps between Riemann surfaces of negative curvature developed by Schoen and Yau [SY] [Yau3] has been very useful in studying the Teichmüller spaces and related problems [Wol1] [Wol2] [Ru] [DDW]. 
In this subsection, we briefly discuss the results in [Co] and [GS]. First, the Mostow strong rigidity in Theorem 2.8.1 can be restated as follows.

Proposition 3.4.5 Let $G, G^{\prime}$ be two connected semisimple Lie groups with trivial center and no compact simple factors. Suppose that $\Gamma \subset G, \Gamma^{\prime} \subset G^{\prime}$ are uniform lattices, $\Gamma$ is irreducible. Suppose furthermore that if $G$ is of rank one, then $G$ is not equal to $S L(2, \mathbb{R})$, or equivalently $\operatorname{dim} G \geq 4$. Then any isomorphism $\pi: \Gamma \rightarrow \Gamma^{\prime}$ extends to an isomorphism $\pi: G \rightarrow G^{\prime}$.

The super-rigidity removes the assumption that the image $\pi(\Gamma)$ is a uniform lattice in $G^{\prime}[\mathrm{Zi}$, Theorem 5.1.2] [Mar, Theorems 2 and 3, pp. 2-3].

Theorem 3.4.6 Let $\mathbf{G}$ be a connected linear semisimple algebraic group defined over $\mathbb{R}$ with $\mathbb{R}$ rank at least 2. Assume that the identity component $\mathbf{G}(\mathbb{R})^{0}$ has no compact factors. Suppose that $k_{\mathfrak{p}}$ is a local field of characteristic 0 , and $\mathbf{H}$ a connected linear algebraic group defined and simple over $k_{\mathfrak{p}}$. Assume that $\pi: \Gamma \rightarrow \mathbf{H}\left(k_{\mathfrak{p}}\right)$ is a homomorphism with $\pi(\Gamma)$ Zariski dense in $\mathbf{H}$. Then one of the following alternatives hold:

1. If $k_{\mathfrak{p}}=\mathbb{R}$ and $\mathbf{H}(\mathbb{R})$ is not compact, then $\pi$ extends to a rational homomorphism $\mathbf{G} \rightarrow \mathbf{H}$ defined over $\mathbb{R}$.

2. If $k_{\mathfrak{p}}=\mathbb{C}$, then either (a) the closure of $\pi(\Gamma)$ in the ordinary topology is compact, or (b) $\pi$ extends to a rational homomorphism $\pi: \mathbf{G} \rightarrow \mathbf{H}$.

3. If $k_{\mathfrak{p}}$ is totally disconnected (i.e., non-archimedean), then $\pi(\Gamma)$ is bounded in $\mathbf{H}\left(k_{\mathfrak{p}}\right)$, i.e., its closure with respect to the natural Hausdorff topology of $\mathbf{H}\left(k_{\mathfrak{p}}\right)$ is compact.

An important consequence of this theorem is the celebrated arithmeticity theorem of irreducible lattices of rank 2 (see [Zi, Theorem 6.1.2] [Mar, Theorem 1, p. 2, Theorem (1'), p. 4]), which together with a result of Prasad [Pr3] show that Proposition 3.4.5 holds when $\Gamma, \Gamma^{\prime}$ are only assumed to be lattices, but not necessarily co-compact. It should be pointed out that if $\mathbf{G}(\mathbb{R})$ is the real locus of a linear connected semisimple algebraic group defined over $\mathbb{R}$ and $\mathbf{G}$ has no compact simple factors, then the Borel density [Zi, Theorem 3.2.5] says that any lattice $\Gamma$ in $\mathbf{G}(\mathbb{R})$ is Zariski dense in $\mathbf{G}$, i.e., $\Gamma$ is dense in $\mathbf{G}$ with respect to the Zariski topology. So the assumption on the Zariski density in Theorem 3.4.6 is natural.

Since the strong Mostow rigidity in Proposition 3.4.5 holds in the rank one case with one exception, it is natural to ask if the super-rigidity holds for lattices in some rank one Lie groups as well.

According to the classification of symmetric spaces of ( $\mathbb{R}$-)rank 1 (see [Mos, §19]), there are four types:

1. the real hyperbolic spaces $\mathbf{H}_{\mathbb{R}}^{n}=S O^{0}(1, n) / S O(n), n \geq 2$;

2. the complex hyperbolic spaces $\mathbf{H}_{\mathbb{C}}^{n}=S U(1, n) / U(n), n \geq 2$;

3. the quaternionic hyperbolic spaces $\mathbf{H}_{\mathbb{H}}^{n}=S p(1, n) / S p(n), n \geq 2$;

4. the (exceptional) Cayley hyperbolic plane $\mathbf{H}_{\mathbb{O}}^{2}=F_{4(-20)} / \operatorname{Spin}(9)$.

It is known [Co, p.166] that the super-rigidity for lattices $\Gamma$ acting on the real and complex hyperbolic spaces, $\Gamma \subset S O^{0}(1, n)$ or $S U(1, n)$, does not hold due to existence of non-arithmetic lattices. In $[\mathrm{Co}]$, it is shown that the archimedean super-rigidity, i.e., $k_{\mathfrak{p}}=\mathbb{R}, \mathbb{C}$, holds for lattices 
in $S p(1, n)$ and $F_{4(-20)}$. It uses the method of harmonic maps initiated in [Si2] (see [JY1] for a survey of the Bochner-Matsushima type formulas and [Mat1] [Ye3]).

The proof of the Mostow strong rigidity theorem using harmonic maps goes in two steps:

1. Let $\psi$ be the map in the Equation 2.8.1. Using the nonpositive curvature of the target manifold, show that there exists a unique harmonic map $\Psi: \Gamma \backslash X \rightarrow \Gamma \backslash X^{\prime}$ in the homotopy equivalence class of the map $\psi$.

2. Using a Bochner type formula, show the vanishing of certain derivatives of $\Psi$ and hence that $\Psi$ is totally geodesic, which in turn implies that $\Psi$ is an isometry up to suitable scaling.

Since the harmonic map $\Psi$ is deformed from $\psi$ (or rather the equivariant quasi-isometry $\varphi$ ), this gives explicitly an isomorphism between the Lie groups $G$ and $G^{\prime}$ in Theorem 2.8.1. The arguments in the other cases are similar, replacing the harmonic maps between $\Gamma \backslash X$ and $\Gamma \backslash X^{\prime}$ by equivariant maps. We briefly outline the arguments in $[\mathrm{Co}]$ and indicate where the assumption that the Lie groups are $S p(1, n)$ or $F_{4(-20)}$ is used.

Let $G=S p(1, n)$ or $F_{4(-20)}$, and $\Gamma \subset G$ a lattice. Let $G^{\prime}$ be a simple noncompact Lie group with trivial center, and $\rho: \Gamma \rightarrow G^{\prime}$ a homomorphism with a Zariski dense image. We need to show that $\rho$ extends to a homorphism $\rho: G \rightarrow G^{\prime}$. There are three steps:

1. There is a finite-energy, $\rho$-equivariant map from $X=G / K$ to $X^{\prime}=G^{\prime} / K^{\prime}$, where $K \subset G$ and $K^{\prime} \subset G^{\prime}$ are maximal compact subgroups.

2. Show that the finite-energy equivariant map can be modified into a finite-energy $\rho$-equivariant harmonic map $f: X \rightarrow X^{\prime}$.

3. On $X$, there exists a parallel differential form $\omega$ such that its annihilator in $\operatorname{End}\left(T_{o} X\right)$ consists of only skew-symmetric endomorphisms, where $T_{o} X$ is the tangent space to $X$ at $o \in X$.

4. The harmonic map $f$ is totally geodesic and the Zariski density of the image of $\Gamma$ implies that $f$ is an isometry between $X$ and $X^{\prime}$, which in turn implies that $\rho$ extends to a homomorphism from $G$ to $G^{\prime}$.

Step (1) follows from [Co, Proposition 2.3] and depends on the fact that the root spaces have sufficiently large dimensions, which is satisfied by $S p(1, n)$ and $F_{4(-20)}$. Step (2) is given in [Co, Theorem 2.1] and works for other groups as well. Step (3) is proved in [Co, Propositions 1.2 and 1.4], and also works for some other higher rank groups [Co, p. 166]. Step (4) is given in [Co, Proposition 3.3] and depends crucially on Step (3) and a new Bochner-type formula, i.e., $D^{*}(d f \wedge \omega)=0$ in [Co, Theorem 3.2].

The arguments in [GS] are similar to the arguments in [Co] once the theory of harmonic maps into Euclidean buildings (or rather $F$-connected complexes) is established. We indicate briefly why in this case the image of the representation of $\Gamma$ is bounded, while in [Co], the representation of $\Gamma$ extends to a isomorphism of the Lie group and hence the image is clearly not bounded.

In fact, let $G=S p(1, n)$ or $F_{4(-20)}$ and $\Gamma \subset G$ a lattice as above, and $\mathbf{G}^{\prime}$ be an almost simple algebraic group defined over a non-archimedean local field $k_{\mathfrak{p}}$. Let $\rho: \Gamma \rightarrow \mathbf{G}^{\prime}\left(k_{\mathfrak{p}}\right)$ be a homomorphism. Then we need to show the image $\rho(\Gamma)$ is a bounded subgroup in $\mathbf{G}^{\prime}\left(k_{\mathfrak{p}}\right)$. Let $\Delta^{B T}\left(\mathbf{G}^{\prime}\right)$ be the Bruhat-Tits building associated with $\mathbf{G}^{\prime}$ (or rather to its simply connected covering group) in $\S 3.2$.

The proof in [GS] follows several steps:

1. The homomorphism $\rho$ induces a finite-energy Lipschitz $\rho$-equivariant map from $X=G / K$ to $\Delta^{B T}\left(\mathbf{G}^{\prime}\right)$. 
2. There is a finite-energy Lipschitz $\rho$-equivariant map of least energy $u: X \rightarrow \Delta^{B T}\left(\mathbf{G}^{\prime}\right)$ whose restriction to small balls at any point is also minimizing, which implies that $u$ is a harmonic map.

3. The map $u$ is called differentiable (or non-singular) at a point $p \in X$ if the local images of $u$ around $p$ are contained in an apartment of $\Delta^{B T}\left(\mathbf{G}^{\prime}\right)$. Show that the set of singular points of $u$ has Hausdorff dimension at most $\operatorname{dim} X-2$.

4. Show that the harmonic map $u$ is constant around the differentiable points and is hence constant.

5. Let $p$ be the unique point in the image $u(X)$. Then the image $\rho(\Gamma)$ is contained in the stabilizer in $\mathbf{G}^{\prime}\left(k_{\mathfrak{p}}\right)$ of the point $p \in \Delta^{B T}\left(\mathbf{G}^{\prime}\right)$, and is hence contained in a parahoric subgroup of $\mathbf{G}^{\prime}\left(k_{\mathfrak{p}}\right)$, which is a bounded subgroup.

Step (1) is proved in [GS, Lemma 8.1] and uses $\operatorname{dim} X \geq 8$. Step (2) is proved in [GS, Theorem 7.1]. Step (3) is proved in [GS, Theorem 6.4], which works for more general $F$-connected complexes.

Step (4) is given in [GS, Theorem 7.4] and is a new feature not present in [Co]. In fact, since the local image is contained in an apartment which can be identified with $\mathbb{R}^{r}$, where $r$ is the $k_{\mathfrak{p}}$-rank of $\mathbf{G}^{\prime}$, the coordinates of the harmonic map $u$ give parallel 1-forms. If they are not zero, they will give local isometric splitting of $X$ with $\mathbb{R}$ as a factor. Since this is impossible, it implies that $u$ must be locally constant.

Remark 3.4.7 The philosophical differences between the original approach of Mostow and the approach here are interesting. In the former case, the quasi-isometry $\varphi$, the lift of $\psi$, is pushed to infinity in $X$ and $X^{\prime}$ to get precise maps, i.e., an isomorphism of the spherical Tits buildings, and a homeomorphism of the maximal Furstenberg boundaries. On the other hand, in the latter case, the quasi-isometry $\varphi$ is deformed locally into an isometry via the theory of harmonic maps. In a sense, the former is a global argument, and the latter is local and does not depend on the theory of spherical Tits buildings. It is also instructive to point out that the idea in $\S 4$ is to scale down or shrink symmetric spaces infinitely to get $\mathbb{R}$-Euclidean buildings. This is another type of global geometry. These remarks confirm the point of view that buildings reflect the global geometry in more than one way.

Remark 3.4.8 After the success of [GS] in applying the theory of harmonic maps into Euclidean buildings, the theory has been generalized to CAT(0)-metric spaces. See [KS1] [KS2] [Jo3] [Bal1] $[\mathrm{JZ}]$.

Remark 3.4.9 The Mostow strong rigidity deals with uniform lattices in semisimple Lie groups. Its generalization to lattices in semisimple $p$-adic Lie groups is given by Prasad in [Pr2]. The proof followed the method of [Mos]. Instead of realizing the spherical Tits building using the geometry at infinity of the symmetric spaces of noncompact type, Euclidean buildings were used to obtain spherical buildings at infinity, which will be explained in the next subsection.

Remark 3.4.10 In [Mar], lattice subgroups in products of real and $p$-adic groups, in particular, $S$-arithmetic subgroups, are studied, and hence the archimedean and non-archimedean places are studied at the same time. Basically, irreducible $S$-arithmetic subgroups of semisimple algebraic groups of total rank greater than or equal to 2 are super-rigid. 


\subsection{Compactification of Euclidean buildings by spherical buildings}

For a connected linear semisimple algebraic group $\mathbf{G}$ defined over a non-archimedean local field $F$, there are two buildings associated with $\mathbf{G}$ : the spherical Tits building $\Delta(\mathbf{G})$ and the Bruhat-Tits Euclidean building $\Delta^{B T}(\mathbf{G})$. These two buildings are closely related. In fact, the former with a suitable topology can be used to compactificatify the latter. This compactification is similar to the geodesic compactification $X \cup X(\infty)$ of a symmetric space $X$ of noncompact type in $\S 2.5$, and is very important for applications to the cohomology of $S$-arithmetic groups in [BoS1].

The construction was given in [BoS1] and also reviewed in [Br1] and [Ro3]. Here we recall the compactification, in particular its topology in a slightly different way, i.e., we view the Euclidean building as a $\mathrm{CAT}(0)$-space $[\mathrm{BH}]$.

As recalled earlier, a CAT(0)-space is a geodesic metric space whose triangles are thinner than the corresponding triangles of the same side lengths in $\mathbb{R}^{2}$. A complete simply connected manifold of nonpositive curvature, i.e., a Hadamard manifold, is a CAT(0)-space, and CAT(0)-spaces are generalizations of Hadamard manifolds to the category of metric spaces.

Let $(\Delta, d)$ be a $\operatorname{CAT}(0)$-space. A ray in $\Delta$ is an isometric embedding $\gamma:[0,+\infty) \rightarrow \Delta$. Two rays $\gamma_{1}, \gamma_{2}$ are called equivalent if

$$
\limsup _{t \rightarrow+\infty} d\left(\gamma_{1}(t), \gamma_{2}(t)\right)<+\infty
$$

Let $\Delta(\infty)$ be the set of equivalence classes of rays in $\Delta$.

Define a conic topology on $\Delta \cup \Delta(\infty)$ as follows: Let $o \in \Delta$ be any basepoint. It is known that every equivalence class $[\gamma]$ of rays contains a unique ray $\gamma$ which starts from $o$, i.e., $\gamma(0)=o$. Define $\gamma(+\infty)$ to be the equivalence class $[\gamma]$. Then we get an extended ray

$$
\gamma:[0,+\infty] \rightarrow \Delta \cup \Delta(\infty) .
$$

For any ray $\gamma$ with $\gamma(0)=o$ and $r>0$ and $\varepsilon>0$, define a neighborhood of $\gamma(+\infty)$ by

$$
U(\gamma(+\infty), r, \varepsilon)=\left\{\gamma^{\prime}(t) \mid \gamma^{\prime} \text { is a ray, } \gamma^{\prime}(0)=0, d\left(\gamma^{\prime}(r), \gamma(r)\right)<\varepsilon, t \in(r,+\infty]\right\} .
$$

When $r$ runs over a sequence of numbers going to infinity, and $\varepsilon$ over a sequence going to 0 , $U(\gamma(+\infty), r, \varepsilon)$ forms a neighborhood basis of $\gamma(+\infty)$ in $\Delta \cup \Delta(\infty)$ (see [BH, pp. 263-264]).

Remark 3.5.1 Let $S(o, r)=\{x \in \Delta \mid d(x, o)=r\}$ be the sphere of radius $r$ with center $o$. Then $\gamma(r), \gamma^{\prime}(r) \in S(o, r)$. For a Hadamard manifold, the spheres $S(o, r)$ are all diffeomorphic for different values of $r$. This implies that in this case, we could only use one fixed $r$ to get a neighborhood basis of $\gamma(+\infty)$. On the other hand, for a general CAT(0)-space, $r$ needs to run over a sequence of numbers going to infinity. This can be seen clearly when $\Delta$ is an infinite tree.

In this topology of $\Delta \cup \Delta(\infty)$, an unbounded sequence $x_{j}$ converges to a boundary point $\gamma(+\infty)$ if and only if the unique ray $\gamma_{j}(t)$ starting from $o$ and passes through $x_{j}$ converges to $\gamma(t)$ uniformly for $t$ in compact subsets.

Proposition 3.5.2 If $\Delta$ is complete, the space $\Delta \cup \Delta(\infty)$ is contractible.

Proof. Let $o \in \Delta$ be a basepoint as above. Then each ray $\gamma$ issuing from $o$ extends continuously to an extended ray $\gamma:[0,+\infty] \rightarrow \Delta \cup \Delta(\infty)$. When $\gamma$ runs over all the rays starting from $o$, the extended ray sweeps out $\Delta \cup \Delta(\infty)$. Now contract $\Delta \cup \Delta(\infty)$ along such each extended rays to the basepoint $o$ using a fixed deformation retract of $[0,+\infty)$ to 0 . It follows easily from the definition of the conic topology that they define a deformation retraction of $\Delta \cup \Delta(\infty)$ to the basepoint $o$. 
Proposition 3.5.3 If a CAT(0)-space $\Delta$ is complete and locally compact, then $\Delta \cup \Delta(\infty)$ is compact, and $\Delta \cup \Delta(\infty)$ is called the geodesic compactification.

Proof. Since $\Delta$ is a geodesic space, the local compactness and completeness imply that it is a proper metric space [Ha, p. 84]. It suffices to show that any unbounded sequence $x_{j}$ in $\Delta$ has a convergent subsequence. Let $\gamma_{j}$ be the unique ray from $o$ which passes through $x_{j}$. The properness of $\Delta$ implies that for any $r>0$, the closed ball $B(o, r)$ of radius $r$ and center $o$ is compact, which in turn implies that there is a subsequence of $\gamma_{j}(t)$ which converges to a ray $\gamma(t)$ uniformly for $t$ in compact subsets. Then the corresponding subsequence of $x_{j}$ converges to $\gamma(+\infty)$.

If $\Delta$ is a Euclidean building, then $\Delta(\infty)$ is the underlying space of a spherical Tits building. The details are given in [BoS1] (see also [Br1] [Ro3]). We comment briefly on the construction. First we define the notion of sectors of apartments of $\Delta$. Let $\Sigma$ be an apartment in $\Delta$ and identify it with $\mathbb{R}^{r}$. Let $W$ the Euclidean Coxeter group, and $\bar{W}$ be its linear part. Then $\bar{W}$ is a finite Coxeter group and divides $\mathbb{R}^{n}$ into finite many simplicial cones and their faces. (In fact, they are the chambers and chamber faces of the finite Coxeter complex associated with $\bar{W}$ ). The corresponding subsets in $\Sigma$ are called the sectors and sector faces in $\Delta$.

For each sector $C$ of $\Delta$, let $\bar{C}$ be the closure of $C$ in $\Delta \cup \Delta(\infty)$. Define

$$
\bar{C}(\infty)=\bar{C} \cap X(\infty) .
$$

Then $\bar{C}(\infty)$ is a closed simplex. Denote its interior by $C(\infty)$, called the simplex at infinity associated with $C$. For any sector face $C_{I}$ of $C$, we can also define a simplex at infinity $C_{I}(\infty)$. Then it is known [Br1, pp. 175-177] that these simplexes at infinity give a partition of $\Delta(\infty)$ and define a spherical Tits building, denoted by $\Delta_{\infty}$.

Remark 3.5.4 Another slightly different construction of the spherical Tits building $\Delta_{\infty}$ was contained in the proof of the Mostow strong rigidity and can be described as follows. Two sectors $C_{1}, C_{2}$ are called equivalent if the Hausdorff distance between them is finite. Similarly we can define equivalent sector faces, and the incidence relation between the equivalence classes of sectors and sector faces. Then the set of equivalence classes of sectors and sector faces form the spherical Tits building at infinity $\Delta_{\infty}$.

If $\Delta$ is the Bruhat-Tits building $\Delta^{B T}(\mathbf{G})$ of a linear connected simply connected algebraic group $\mathbf{G}$ defined over a local field $k_{\mathfrak{p}}$, then the spherical building $\Delta_{\infty}$ is exactly the spherical Tits building $\Delta(\mathbf{G})$. In this case, the subset topology on $\Delta(\infty)$ induces a topology on $\Delta(\mathbf{G})$ and is different from the canonical topology of $\Delta(\mathbf{G})$ defined by the Tits metric. For example, when the rank of $\mathbf{G}$ over $k_{\mathfrak{p}}$ is equal to 1 , the Tits metric defines the discrete topology on $\Delta(\mathbf{G})$. On the other hand, the topology on $\Delta(\infty)$ is not discrete; in fact, it is a compact space homeomorphic to the Cantor set.

The topology of $\Delta(\mathbf{G})$ induced from the subset topology of $\Delta^{B T}(\mathbf{G})(\infty)$ can be described as follows. Since $\mathbf{G}\left(k_{\mathfrak{p}}\right)$ acts isometrically on $\Delta^{B T}(\mathbf{G})$, it preserves the equivalence classes of rays and acts continuously on the compactification $\Delta^{B T}(\mathbf{G}) \cup \Delta(\mathbf{G})$, and hence on $\Delta(\mathbf{G})$ as well. Let $\sigma$ be a simplex in $\Delta(\mathbf{G})$ of the top dimension, i.e., of dimension $r-1$. Then the map

$$
\mathbf{G}\left(k_{\mathfrak{p}}\right) \times \sigma \rightarrow \Delta(\mathbf{G})
$$

is surjective. The subset topology of $\sigma$ is clearly equal to the one given by the simplicial structure. In [BoS1], the topology of $\Delta(\mathbf{G})$ is given by the quotient topology of $\mathbf{G}\left(k_{\mathfrak{p}}\right) \times \sigma$ under the above map and is shown to be compact and Hausdorff. The above discussions show that this topology is as fine as the topology induced from $\Delta^{B T}(\infty)$. Since both topologies are compact and Hausdorff, they agree. 
Remark 3.5.5 The topology on $\Delta(\mathbf{G})$ naturally makes it a topological building according to Definition 2.7.1. In fact, the set $\Delta_{1}$ of the vertexes is equal to the disjoint union of $\mathbf{G}\left(k_{\mathfrak{p}}\right) / \mathbf{P}_{m}\left(k_{\mathfrak{p}}\right)$, $m=1, \cdots, r$, each is given with the quotient topology and is a compact Hausdorff space, where $\mathbf{P}_{1}, \cdots, \mathbf{P}_{r}$ are representatives of conjugacy classes of maximal parabolic subgroups. For any $s=$ $2, \cdots, r, \Delta_{s}$ is a finite union of $\mathbf{G}\left(k_{\mathfrak{p}}\right) / \mathbf{P}\left(k_{\mathfrak{p}}\right)$, where $\mathbf{P}$ run over some representatives of conjugacy classes of parabolic subgroups of $\mathbf{G}$ of rank $s$. With the quotient topology, $\mathbf{G}\left(k_{\mathfrak{p}}\right) / \mathbf{P}\left(k_{\mathfrak{p}}\right)$ is clearly compact and Hausdorff. Since the map $\Delta_{s} \rightarrow\left(\Delta_{1}\right)^{s}$ is continuous and injective, and all the spaces are compact and Hausdorff, it is clear that the image is closed. This gives the structure of a topological building on $\Delta(\mathbf{G})$.

\subsection{Applications to S-arithmetic groups}

In this subsection, we briefly recall two applications of the Bruhat-Tits buildings to $S$-arithmetic groups. The first concerns the cohomology groups, and the second one the integral Novikov conjectures.

First we define $S$-arithmetic subgroups. Let $k$ be a global field, i.e., either a number field, a finite extension of $\mathbb{Q}$, or the function field of a smooth projective curve over a finite field, for example $\mathbb{F}_{p}(t)$, where $p$ is a prime.

Let $S$ be a finite set of places of $k$ including the set $S_{\infty}$ of all infinite places. Let $S_{f}=S-S_{\infty}$ be the subset of finite places. For each finite place $\mathfrak{p}$, let $\nu_{\mathfrak{p}}$ be the corresponding valuation. Define the ring $\mathcal{O}_{S}$ of $S$-integers in $k$ by

$$
\mathcal{O}_{S}=\left\{x \in k \mid \nu_{\mathfrak{p}}(x) \geq 0, \mathfrak{p} \notin S\right\} .
$$

Let $\mathbf{G} \subset G L(n)$ be a linear algebraic group defined over $k$. A subgroup $\Gamma$ of $\mathbf{G}(k)$ is called an $S$-arithmetic subgroup if it is commensurable with $\mathbf{G}\left(\mathcal{O}_{S}\right)=\mathbf{G}(k) \cap G L(n, k)$.

Assume that $\mathbf{G}$ is semisimple. Then $\Gamma$ is a co-finite discrete subgroup in

$$
G_{S}=\prod_{\mathfrak{p} \in S} \mathbf{G}\left(k_{\mathfrak{p}}\right)
$$

where for $\mathfrak{p} \in S_{f}, k_{\mathfrak{p}}$ is the completion of $k$ with respect to the norm associated with the valuation $\nu_{\mathfrak{p}}$, and for $\mathfrak{p} \in S_{\infty}, k_{\mathfrak{p}}$ is the completion of $k$ with respect to the associated archmedean norm. It is also known that $\Gamma$ is uniform if and only if the $k$-rank $r$ of $\mathbf{G}$ is equal to 0 .

Proposition 3.6.1 (1) When $k$ is a number field, any $S$-arithmetic subgroup $\Gamma$ admits a torsion free subgroup of finite index. (2) When $k$ is a function field, if the rank $r=0$, any $S$-arithmetic subgroup admits a torsion free subgroup of finite index; on the other hand, if the rank $r>0, \Gamma$ does not admit any torsion free subgroup of finite index.

(1) is proved in $\left[\mathrm{Se} 2\right.$, p. 126, $\left.\left(a_{3}\right)\right]$ (see also [Br1, p. 196]). The first part of $(2)$ is proved in $[\mathrm{Se} 2$, p. 126, Case (b)]. The second part follows from the fact that any subgroup of finite index of $\Gamma$ contains unipotent elements which are torsion since the characteristic of $k$ is positive.

An important ingredient in studying cohomology groups and related cohomological finiteness of discrete subgroups $\Gamma$ is finding or constructing good models of $K(\Gamma, 1)$-spaces (see [Br3])

From now on, we assume that $\mathbf{G}$ is semisimple. When $S=S_{\infty}, \Gamma$ is an arithmetic subgroup, and $G_{S}$ is a Lie group. Let $X_{\infty}=G_{S} / K$ be the associated symmetric space of noncompact type. Then $\Gamma$ acts properly on $X_{\infty}$. If $\Gamma$ is torsion free, then $\Gamma \backslash X_{\infty}$ is a $K(\Gamma, 1)$-space. 
Otherwise, for every $\mathfrak{p} \in S_{f}$, let $X_{\mathfrak{p}}$ be the Bruhat-Tits building of $\mathbf{G}$ when considered as an algebraic group defined over $k_{\mathfrak{p}}$. Denote the symmetric space associated with $S_{\infty}$ by $X_{\infty}$ as before. Set

$$
X_{S}=X_{\infty} \times \prod_{\mathfrak{p} \in S_{f}} X_{\mathfrak{p}}
$$

Then $\Gamma$ acts properly on $X_{S}$, and hence the stabilizer in $\Gamma$ of any point in $X_{S}$ is finite. If $\Gamma$ is torsion free, then $\Gamma$ acts freely on $X_{S}$. Since $X_{\infty}$ and each $X_{\mathfrak{p}}$ is contractible, this implies that $\Gamma \backslash X_{S}$ is a $K(\Gamma, 1)$-space in this case.

When the rank $r>0, \Gamma \backslash X_{S}$ is not compact and hence is not a finite $C W$-complex, i.e., not a finite $K(\Gamma, 1)$-space. If $k$ is a number field, we can enlarge $X_{S}$ to

$$
\bar{X}_{S}^{B S}=\bar{X}_{\infty}^{B S} \times \prod_{\mathfrak{p} \in S_{f}} X_{\mathfrak{p}},
$$

where $\bar{X}_{\infty}^{B S}$ is the Borel-Serre partial compactification in $\S 2.12$. (We can use the functor of restriction of scalars to replace $\mathbf{G}$ by an algebraic group defined over $\mathbb{Q}$ so that the construction in $\S 2.12$ applies). Then $\Gamma \backslash \bar{X}_{S}^{B S}$ is compact and can be shown to have the structure of finite CWcomplex. Since the inclusion $\Gamma \backslash X_{S} \rightarrow \Gamma \backslash \bar{X}_{S}^{B S}$ is a homotopy equivalence, this gives a concrete finite $K(\Gamma, 1)$-space.

The $\Gamma$-action on $X_{S}$ and $\bar{X}_{S}^{B S}$ and their quotients have played an important and fundamental role in understanding cohomology groups and other related finiteness properties of $\Gamma$. The compactification $\Delta^{B T}(\mathbf{G}) \cup \Delta(\mathbf{G})$ discussed in the previous subsection is also crucial in such applications, which are discussed in great detail in surveys [Se2] [Br1, Chap. VII] [Ro2, §10] [RS] (see also the original paper $[\mathrm{BS} 1])$.

Remark 3.6.2 Cohomological finiteness properties of $S$-arithmetic subgroups of algebraic groups defined over a global field depend in an essential way on whether the field is a number field or a function field, i.e., the function field of a smooth curve over a finite field. In the latter case, the many different kinds of cohomological finiteness properties of $S$-arithmetic subgroups are not well understood. See [Abe1] [Abe2] [AbA] [Beh1] [Beh2] [Bux] and [Br1, Chap. VII, §4]. In all these works, actions on Bruhat-Tits buildings or suitable subcomplexes play an important role.

Remark 3.6.3 As we pointed out in Remark 3.5.5, the boundary $\Delta(\mathbf{G})$ in the compactification $\Delta^{B T}(\mathbf{G}) \cup \Delta(\mathbf{G})$ is a topological spherical Tits building rather than a usual spherical Tits building. Besides the application to the problem of rank rigidity and the rigidity of isoparametric submanifolds, this also confirms our point of view that in applications to geometry and topology, the topological buildings are more natural.

In the rest of this subsection, we discuss an application to the integral Novikov conjectures for $S$-arithmetic subgroups $\Gamma$.

To explain this, we start with the original Novikov conjecture on the homotopy invariance of higher signatures (see [FRR] for precise statements and history of the Novikov conjectures). The Hirzebruch index theorem says that the signature of a compact oriented manifold $M$ of dimension $4 n$ is equal to the evaluation of the Hirzebruch class $\mathcal{L}_{M}$ on the fundamental class, which implies that the latter is an oriented homotopy invariant. Novikov introduced higher signatures by the evaluation of $\mathcal{L}_{M}$ cupped with some cohomology classes pulled back from a $K(\pi, 1)$-space, where $\pi=\pi_{1}(M)$, which are called higher signatures, and conjectured that they are homotopy invariant. 
This original Novikov conjecture is equivalent to the rational injectivity of the assembly map in the surgery (or $L$-) theory, and the (integral) injectivity of the assembly map in the $L$-theory is called the integral Novikov conjecture, which is an important step towards computing the $L$-groups, the image groups of the assembly map. Similarly, there are also assembly maps for the (topological) $K$-groups of $C^{*}$-algebras, in particular the reduced group $C^{*}$-algebras which are the $\ell^{2}$-completions of group algebras $\mathbb{C}[\Gamma]$ of groups $\Gamma$, and for the algebraic $K$-groups of the group ring $\mathbb{Z}[\Gamma]$ of groups $\Gamma$. In each theory, the rational injectivity of the assembly map is called the Novikov conjecture, and the injectivity of the assembly map is called the integral Novikov conjecture in that theory.

A particularly interesting class of groups $\Gamma$ with discrete topology is the class of discrete subgroups of Lie groups. For torsion free arithmetic subgroups $\Gamma$ of linear algebraic groups, the integral Novikov conjectures were proved in [J2] by showing that arithmetic subgroups have finite asymptotic dimension (see [J2] for a brief history about the integral Novikov conjectures for such discrete subgroups). It should be emphasized that the torsion free assumption is in general necessary for the integral Novikov conjectures.

The next natural and important class of groups is the class of $S$-arithmetic subgroups of linear algebraic groups defined over global fields. They are in general not contained in real Lie groups as discrete subgroups.

To prove the integral Novikov conjecture for them, we use the following general result of Carlsson and Pedersen $[\mathrm{CP}]$.

Proposition 3.6.4 Suppose that $\Gamma$ has a finite $K(\Gamma, 1)$-space, denoted by $B \Gamma$, and the universal cover $E \Gamma$ of $B \Gamma$ has a contractible, metrizable $\Gamma$-compactification $\overline{E \Gamma}$ such that the action of $\Gamma$ on $\overline{E \Gamma}$ is small at infinity. Then the integral Novikov conjecture holds for $\Gamma$.

In the above proposition, by a small action at infinity we mean that for any compact subset $K \subset E \Gamma$, if $g_{j} K$ has an accumulation near $z \in \partial \overline{E \Gamma}$, then the whole set $g_{j} K$ is contained in small neighborhoods of $z$.

A typical example is when $\Gamma$ is the fundamental group of a closed nonpositively curved manifold $M$. Then we can take $B \Gamma=M$, and $E \Gamma$ the universal covering $\tilde{M}$. Since $\tilde{M}$ is a Hadamard manifold, it admits the geodesic compactification $\tilde{M} \cup \tilde{M}(\infty)$, and the $\Gamma$-action on $\tilde{M}$ is small.

As discussed before, for a torsion free $S$-arithmetic subgroup $\Gamma$ of a connected linear semisimple algebraic group defined over a global field $k$, we can take $\Gamma \backslash X_{S}$ to be a not necessarily finite $B \Gamma$. When the rank $r$ is equal to zero, it can be shown to be a finite $K(\Gamma, 1)$-space. Now $E \Gamma=X_{S}$. Since $X_{\infty}$ is nonpositively curved and hence a complete CAT(0)-space, and each Bruhat-Tits building $X_{\mathfrak{p}}$ is also a complete CAT(0)-space, this follows that $X_{S}$ is a complete CAT(0)-space. By Proposition 3.5.2 and Proposition 3.5.3, $X_{S}$ admits a contractible compactification $X_{S} \cup X_{S}(\infty)$. Since $\Gamma$ acts on the interior $X_{S}$ with a compact quotient and the compactification $X_{S} \cup X_{S}(\infty)$ is defined in terms of equivalence classes of rays, it can be shown easily that the extended action on $X_{S} \cup X_{S}(\infty)$ is small at infinity. By Proposition 3.6.4, we obtain the following result [J1].

Proposition 3.6.5 If the rank $r$ of a semisimple linear algebraic group $\mathbf{G}$ over $k$ is equal to zero, then the integral Novikov conjecture in K-theory and L-theory holds for any torsion free $S$-arithmetic subgroup of $\mathbf{G}$.

Perhaps it should be pointed out that the existence of a finite or even finite-dimensional $B \Gamma$ implies that $\Gamma$ is torsion free. By the earlier discussions in this subsection, when $k$ is a function field, torsion free $S$-arithmetic subgroups exist only in the case when the rank $r=0$, and hence the result in the proposition is optimal in this case. 
On the other hand, when $k$ is a number field, the assumption that $r=0$ might be removed. But the general method in Proposition 3.6.4 can not be applied directly. In fact, when $r>0$, we can take the compactification $\Gamma \backslash \bar{X}_{S}^{B S}$ as a finite $K(\Gamma, 1)$-space and $\bar{X}_{S}^{B S}$ as $E \Gamma$. To apply Proposition 3.6.4, we need to construct a small compactification of the partial compactification $\bar{X}_{S}^{B S}$. This does not seem to be possible. We need a generalized version of Proposition 3.6.4 by Carlsson-Pedersen with weaker conditions on $E \Gamma$. See $[\mathrm{Gol}]$ for the statement of this result and an application to arithmetic subgroups of $\mathbb{R}$-rank 1 semisimple algebraic groups.

Remarks 3.6.6 After a preliminary version of this paper was written, the author has proved a generalized integral Novikov conjecture in both L- and K-theories for all S-arithmetic subgroups, which may contain torsion elements, of $\mathbf{G}$ of rank $r=0$ over a global field $k$ (see [J1]), using the method in [Rose].

It is also recently proved in [J4] that all torsion-free S-arithmetic groups $\Gamma$ of a semisimple linear algebraic group $\mathbf{G}$ over a number field $k$, without the rank zero restriction, has finite asymptotic dimension and finite $B \Gamma$-spaces; hence the integral Novikov conjecture in both L- and K-theories holds for them. It is not known if the generalized integral Novikov conjecture holds if the Sarithmetic groups contain torsion elements.

\section{$4 \quad \mathbb{R}$-trees and $\mathbb{R}$-buildings}

The Bruhat-Tits buildings of semisimple algebraic groups defined over local fields are simplicial complexes and locally compact. In this section, we recall $\mathbb{R}$-trees and $\mathbb{R}$-Euclidean buildings. They are analogues of the Bruhat-Tits buildings for algebraic groups over fields with non-discrete valuations but not simplicial complexes or locally compact topological spaces. They also arise naturally in the study of global geometry of symmetric spaces of noncompact type and the Euclidean buildings. In fact, $\mathbb{R}$-trees have been used crucially in reproofs of some fundamental results of Thurston's geometrization program of three dimensional manifolds [Th1] [Th2] [Th2], and $\mathbb{R}$-Euclidean buildings were used to prove the Margulis conjecture on quasi-flats in symmetric spaces [KL2].

First we discuss $\mathbb{R}$-trees as metric spaces. Then we show how to construct them from $\Lambda$-valued metric spaces, where $\Lambda$ is an ordered abelian group contained in $\mathbb{R}$, and $S L(2, F)$ where $F$ is a field with non-discrete valuation. $\mathbb{R}$-trees have many applications in topology, and we mention only one sample result from [MS2]. $\mathbb{R}$-Euclidean buildings are more complicated and can not be easily described in terms of $B N$-pairs; and we follow the method in [BT1, §7.4] to construct them for algebraic groups. Finally we consider their applications to quasi-isometric rigidity of symmetric spaces.

\subsection{Definition of $\mathbb{R}$-trees and basic properties}

$\mathbb{R}$-trees were first introduced by Tits [Ti2], and a self-contained description was given in [MS1]. Surveys about $\mathbb{R}$-trees and their applications are given in [Mor1] [Sh]. We will recall some applications in the next subsection.

Definition 4.1.1 A metric space $(T, d)$ is called an $\mathbb{R}$-tree if every pair of points $p, q \in M$ is connected by a unique arc $c:[0, \ell] \rightarrow M$, where $\ell=d(p, q)$, such that (1) $c(0)=p, c(\ell)=q$; (2) the map $c$ is an isometric embedding.

The image of $c$ in $T$ is called a segment. Clearly, if two segments $c_{1}, c_{2}$ only intersect at one end point, their union is also a segment. The intersection of any two segments is either a point or a segment. These properties characterize $\mathbb{R}$-trees among geodesic metric spaces. 
Roughly speaking, $\mathbb{R}$-trees have two characteristic properties: (1) they are 1-dimensional, (2) they don't contain any loops.

A usual (simplicial) tree with a linear distance function on every edge is clearly a $\mathbb{R}$-tree. To explain the difference between simplicial trees and general $\mathbb{R}$-trees, we note if we normalize the edge lengths of a simplicial tree to be equal to 1, then the set of vertexes (or nodes or branch points) of the tree is a discrete subset. On the other hand, in a general $\mathbb{R}$-tree which arises in topology and geometry, the set of branch points is dense or even equal to the whole tree.

Since $\mathbb{R}$-trees are not 1-dimensional simplicial complexes in general, we could not completely describe them usually by specifying vertexes and edges between them. On the other hand, we can start with specifying the collection of vertexes and a distance on them which measures the lengths of the edges connecting them and hence determines the edges in some sense.

To make this more precise and rigorous, we briefly recall the construction of $\mathbb{R}$-trees [MS1]. Let $\Lambda$ be an ordered abelian group, for example, $\mathbb{R}, \mathbb{Z}$ and $\mathbb{Q}$.

Definition 4.1.2 $A \Lambda$-metric space is a pair $(X, d)$, where $X$ is a set and $d$ is a function $d$ : $X \times X \rightarrow \Lambda$ satisfying the following conditions: for all $x, y, z \in X$, (1) $d(x, y) \geq 0$, (2) $d(x, y)=0$ if and only if $x=y$, (3) $d(x, y)=d(y, x)$, and (4) $d(x, z) \leq d(x, y)+d(y, z)$.

Clearly, $\Lambda$ is a $\Lambda$-metric space with the metric $d(x, y)=\max (x-y, y-x)$. A closed segment in a $\Lambda$-metric space is a subset in $X$ isometric to a closed interval $\left[\lambda_{1}, \lambda_{2}\right]=\left\{\lambda \in \Lambda \mid \lambda_{1} \leq \lambda \leq \lambda_{2}\right\}$.

Then a $\Lambda$-tree is defined to be a $\Lambda$-metric space $(T, d)$ satisfying the following conditions:

1. Every two points in $T$ are the end points of a unique closed segment;

2. if two closed segments in $T$ have a common endpoint, then their intersection is a closed segment;

3. for $a, b, c \in \Lambda$ with $a \leq c \leq b$, if $\mu:[a, b] \rightarrow T$ is a map such that $\left.\mu\right|_{[a, c]}$ and $\left.\mu\right|_{[b, c]}$ are parametrized segments and $\mu([a, c]) \cap \mu([c, b])=\{\mu(c)\}$, then $\mu$ is a parametrized segment.

When $\Lambda=\mathbb{R},(2)$ is automatically satisfied [MS1, Lemma II.1.1], and (3) corresponds to the condition of containing no loops, and hence an $\mathbb{R}$-tree defined here is also an $\mathbb{R}$-tree in the sense defined earlier.

When $\Lambda$ is a subgroup of $\mathbb{R}$, then we can take a $\Lambda$-tree as the set of vertexes and fill in suitable edges to get an $\mathbb{R}$-tree [MS1, Theorem II.1.9].

Proposition 4.1.3 Assume that $\Lambda \subseteq \mathbb{R}$. Then every $\Lambda$-tree $T$ can be embedded isometrically in a unique $\mathbb{R}$-tree $T_{\mathbb{R}}$ such that (a) $T_{\mathbb{R}}$ is a complete metric space, (b) the union of all closed segments in $T_{\mathbb{R}}$ with endpoints in $T$ is a dense subset of $T_{\mathbb{R}}$. If $\Lambda$ is dense in $\mathbb{R}$, then $T$ is dense in $T_{\mathbb{R}}$. If $\Lambda$ is discrete and generated by $\ell>0$, then $T_{\mathbb{R}}$ can be identified with a simplicial tree whose set of vertexes is equal to $T$ and whose edges have a common length $\ell$.

If $\Lambda$ is a dense subset of $\mathbb{R}$, this proposition may give an $\mathbb{R}$-tree which is not a simplicial tree. To illustrate this, let $F=\overline{\mathbb{Q}_{p}}$ be the algebraic closure of the $p$-adic field, where $p \geq 2$ is a prime number. The discrete valuation of $\mathbb{Q}_{p}$ extends to a non-discrete valuation of $F$. Let $\Lambda$ be the subgroup of $\mathbb{R}$ generated by the values of this valuation. Let $\mathcal{O}$ be the valuation ring of $F$. Then there there is a $\Lambda$-tree $T$ associated to the simple algebraic group $S L(2, F)$ [MS1, II. 3] (see [Mor4] also for the higher dimensional case).

In fact, a lattice in $F^{2}$ is a finitely generated $\mathcal{O}$-module in $F^{2}$ which spans $F^{2}$. Two lattices are called equivalent if one is the multiple of another by a nonzero element of $F$. Then points of 
the $\Lambda$-tree are given by equivalence classes of lattices in $F^{2}$. To define a distance between any two such equivalence classes, we note that for any two lattices $L$ and $L^{\prime}$, there exists a unique lattice $L_{0}^{\prime}$ which is equivalent to $L^{\prime}$ and contained in $L$ such that $L / L_{0}^{\prime}$ is isomorphic to $\mathcal{O} / \beta \mathcal{O}$ for some $\beta \in \mathcal{O}$ [MS1, Proposition II.3.5]. Then the distance between the equivalence classes $[L]$ and $\left[L^{\prime}\right]$ is equal to the valuation of $\beta$.

On the other hand, simplicial trees also arise from the construction in this proposition. For example, take the $\Lambda$-metric space $T$ to be equal to $\Lambda$. Then the associated $\mathbb{R}$-tree is equal to $\mathbb{R}$.

As will be seen later, $\mathbb{R}$-trees also occur naturally as limit objects in topology and geometry.

\subsection{Applications of $\mathbb{R}$-trees in topology}

The original application of the theory of $\mathbb{R}$-trees [MS1] is to give an alternative proof of compactness of the space of hyperbolic structures of certain three dimensional manifolds in [Thu1] (see Theorem 4.2.2 below), which plays an important role in Thurston's geometrization program (Theorem 4.2.1). This theory has also been used in many other problems in topology and geometry.

Let $\Gamma$ be a torsion free finitely generated group. An $n$-dimensional hyperbolic structure on $\Gamma$ is a pair $(N, \varphi)$ such that $N$ is an $n$-dimensional hyperbolic manifold and $\varphi: \Gamma \rightarrow \pi_{1}(N)$ is an isomorphism. Each hyperbolic structure corresponds to a faithful representation $\rho: \Gamma \rightarrow S O^{0}(1, n)$ with discrete image.

Two hyperbolic structures $\left(N_{1}, \varphi_{1}\right)$ and $\left(N_{2}, \varphi_{2}\right)$ on $\Gamma$ are equivalent if there is an isometry between $N_{1}, N_{2}$ which commutes with the isomorphisms $\varphi_{1}, \varphi_{2}$. Two representations $\rho_{1}, \rho_{2}$ of $\Gamma$ in $S O^{0}(1, n)$ are equivalent if and only if they are conjugate. This implies that the set $\mathcal{H}^{n}(\Gamma)$ of equivalence classes of hyperbolic structures on $\Gamma$ is naturally identified with the set of conjugacy classes of faithful representations of $\Gamma$ in $S O^{0}(1, n)$ with discrete images. This identification gives a natural topology on $\mathcal{H}^{n}(\Gamma)$.

If $\Gamma$ is the fundamental group of an $n$-dimensional hyperbolic manifold $M$ of finite volume with $n \geq 3$, then the Mostow strong rigidity (Theorem 2.8.1) implies the hyperbolic structure on $M$ is unique and $\mathcal{H}^{n}(\Gamma)$ consists of only one point. In particular, $\mathcal{H}^{n}(\Gamma)$ is compact. Hence, the compactness of $\mathcal{H}^{n}(\Gamma)$ is a weak version of the Mostow rigidity.

It is well-known that for $n=2, \mathcal{H}^{n}(\Gamma)$ is noncompact. If $\Gamma$ is the fundamental group of a compact hyperbolic surface, then for $n \geq 3, \mathcal{H}^{n}(\Gamma)$ contains $\mathcal{H}^{2}(\Gamma)$ and is noncompact. In fact, the closure of $\mathcal{H}^{2}(\Gamma)$ in $\mathcal{H}^{n}(\Gamma)$ is noncompact and hence $\mathcal{H}^{n}(\Gamma)$ is noncompact. The three dimensional hyperbolic manifolds in $\mathcal{H}^{3}(\Gamma)$ which belong to the subset $\mathcal{H}^{2}(\Gamma)$ have infinite volume and have natural compactifications which are manifolds with nonempty boundaries; in fact, the boundary consists of two Riemann surfaces. In general, if $\Gamma$ is the fundamental group of a hyperbolic manifold of infinite volume (of finite geometric type), then $\mathcal{H}^{n}(\Gamma)$ might be noncompact. As explained below, manifolds with boundary occur naturally in studying three dimensional topology.

An important result of the Thurston geometrization program is the following hyperbolization Theorem of Thurston. For simplicity, we state only the version for closed manifolds (see [Mor3, pp. 51-52] [Kap, Preface] [Ot1] for general results).

Theorem 4.2.1 If $M$ is a compact orientable irreducible atoroidal Haken 3-manifold without boundary, then $M$ admits a complete hyperbolic structure of finite volume.

In this theorem, a 3-manifold $M$ is called irreducible if every sphere $S^{2} \subset M$ bounds a 3-ball in $M$. A closed 3-manifold $M$ is called atoroidal if it is irreducible and every map from the torus $T^{2}$ to $M$ has a nontrivial kernel on the fundamental group. A 3-manifold $M$ is called Haken if it contains a properly embedded incompressible surface $S$ not equal to the two sphere, where by 
incompressibility, we mean that the induced map $\pi_{1}(S) \rightarrow \pi_{1}(M)$ is injective (see [Mor3] and [Kap] for these definitions in the general case).

In proving this, the following compactness result [Thu1, Theorem 1.2] is crucial.

Theorem 4.2.2 If $\Gamma$ is the fundamental group of a three dimensional acylindrical manifold $M$, then $\mathcal{H}^{n}(\Gamma)$ is compact.

In the above theorem, a 3-manifold $M$ is called acylindrical if it does not contain an incompressible torus or essential annuli.

The proof of Theorem 4.2.1 is basically divided in two cases: (1) the manifold $M$ contains an incompressible surface which is not a fiber of a fibration over the circle, (2) the manifold $M$ is a surface bundle over the circle.

In Case (1), let $S \subset M$ be an incompressible surface that separates $M$ into two compact manifolds $M_{1}, M_{2}$ with boundary. Then $S$ is contained in the boundaries of $M_{1}, M_{2}$. Denote the image of $S$ in them by $S_{1}$ and $S_{2}$. By induction, assume that both $M_{1}, M_{2}$ have hyperbolic metrics. The problem is to deform the metrics in the space of hyperbolic metric so that the metrics in neighborhoods of $S_{1}$ and $S_{2}$ match and can be glued into a hyperbolic metric on $M$. Since the theory of fixed points of maps on compact spaces is used, the compactess of $\mathcal{H}^{n}\left(\pi\left(M_{i}\right)\right), i=1,2$, is crucial. See [Kap, Preface] [Mor3] for detailed outline of the arguments.

In Case (2), $M$ is a surface bundle over $S^{1}$ and hence a mapping torus of a homeomorphism of a surface. In the proof, an important ingredient is the double limit theorem. After the original proof of Thurston, Otal [Ot2] gave an alternative proof for the generic case which depends crucially on the theory of group actions on $\mathbb{R}$-trees developed by Morgan-Shalen and others. To state the double limit theorem and indicate how $\mathbb{R}$-trees enter, we quote a part of the Review of [Ot2] by L.Mosher in Math Reviews:

" To state the double limit theorem, consider the space $\mathcal{Q F}(S)$ of quasi-Fuchsian representations of $\pi_{1}(S)$ into Isom $\left(\mathbf{H}^{3}\right)$ up to conjugacy (to simplify matters, assume $S$ is closed). A theorem of Ahlfors and Bers parameterizes the space $\mathcal{Q F}(S)$ as the product of two copies of the Teichmüller space $\mathcal{T}(S)$ of $S$. In order to investigate what happens to a representation as the parameters in $\mathcal{T}(S)$ go to infinity, Thurston used a compactification $\overline{\mathcal{T}}(S)=\mathcal{T}(S) \cup \mathcal{P} \mathcal{M L}(S)$, where $\mathcal{P} \mathcal{M L}(S)$ is the space of projective classes of measured laminations on $S$. Consider a sequence $\rho_{i}=\left(\sigma_{i}^{+}, \sigma_{i}^{-}\right)$in $\mathcal{Q F}(S)$ such that the sequences $\left(\sigma_{i}^{+}\right),\left(\sigma_{i}^{-}\right) \in \mathcal{T}(S)$ converge, respectively, to the projective classes of two measured geodesic laminations $\lambda^{+}, \lambda^{-}$on $S$. If these two laminations fill up the surface, that is, if each component of $S-\left(\lambda^{+} \cup \lambda^{-}\right)$is simply connected, then the double limit theorem says that $\rho_{i}$ has a subsequence converging to some representation of $\pi_{1}(S) \rightarrow \operatorname{Isom}\left(\mathbf{H}^{3}\right)$.

"To sketch the author's new proof of the double limit theorem, suppose $\rho_{i}$ has no convergent subsequence. Applying a theorem of Morgan and Shalen, from the degenerating sequence of representations $\rho_{i}$ one extracts an action of $\pi_{1}(S)$ on a real tree $\mathcal{T}$, with small arc stabilizers. By a theorem of Skora [Sk], the action of $\pi_{1}(S)$ on $\mathcal{T}$ is dual to a measured geodesic lamination $\lambda$ on $S$. The author now studies "realizations" of geodesic laminations in real trees, leading to the conclusion that (essentially) the only geodesic laminations on $S$ which are not realizable on $\mathcal{T}$ are those which do not cross $\lambda$. Since $\lambda^{-}, \lambda^{+}$fill up $S$, at least one of them crosses $\lambda$, and so is realizable on $\mathcal{T}$. If, say, $\lambda^{-}$is realizable on $\mathcal{T}$, then for simple closed curves $\gamma \subset S$ that approximate $\lambda^{-}$in $\mathcal{P} \mathcal{M L}(S)$, one obtains an estimate for $l_{\rho_{i}}(\gamma)$, the translation length of $\gamma$ with respect to the quasi-Fuchsian representation $\rho_{i}$. This is shown to contradict Ahlfors' estimate ${ }^{2}$, which compares $l_{\rho_{i}}(\gamma)$ to $l_{\sigma_{i}^{-}}(\gamma)$, the translation length of $\gamma$ with respect to the hyperbolic structure $\sigma_{i}^{-}$."

\footnotetext{
${ }^{2}$ It probably should be Bers instead of Ahlfors
} 

$18]$.

For more detailed outlines of the proof and other discussions, see [Ot2] and also [Kap, Chap.

In a series of three papers by Morgan and Shalen [MS1] [MS2] [MS3], they gave an alternative proof of Theorem 4.2.2 using $\mathbb{R}$-trees and characterizations of $\Gamma$-actions on them. Since the theory works similarly for all $n \geq 3$, we discuss the general case of $\mathbf{H}^{n}, n \geq 3$, below.

The basic steps are as follows:

1. If $\mathcal{H}^{n}(\Gamma)$ is noncompact, then unbounded sequences will lead to some actions of $\Gamma$ on $\mathbb{R}$-trees.

2. Show that such actions on $\mathbb{R}$-trees will lead to splittings of $\Gamma$. Therefore, if such splittings of $\Gamma$ are excluded, the space $\mathcal{H}^{n}(\Gamma)$ is compact.

The $\mathbb{R}$-trees appear in the boundary of the Morgan-Shalen compactification of $\mathcal{H}^{n}(\Gamma)$, which is defined as follows. Let $\rho: \Gamma \rightarrow S O^{0}(1, n)$ be a faithful representation with discrete image. For each $\gamma \in \Gamma$, we define a translation length as follows: if $\rho(\gamma)$ is parabolic, define its translation length to be zero; if it is hyperbolic, $\rho(\gamma)$ fixes a unique geodesic, called the axis of translation of $\rho(\gamma)$, in $\mathbf{H}^{n}$ and acts as translation on it, and define its translation length $\ell(\rho(\gamma))$ to be the translation length on the geodesic invariant under it. Let $\mathcal{C}$ be the conjugacy classes in $\Gamma$. Then each $\rho$ defines a translation length function

$$
\ell_{\rho}: \mathcal{C} \rightarrow[0,+\infty)^{\mathcal{C}}, \quad \gamma \mapsto \ell(\rho(\gamma))
$$

Let $P(\mathcal{C})=\mathbb{R}^{\mathcal{C}} / \mathbb{R}^{\times}$be the projective space based on $\mathcal{C}$. Then $\ell_{\rho}$ defines a point in $P(\mathcal{C})$, and this induces a map

$$
\Theta: \mathcal{H}^{n}(\Gamma) \rightarrow P(\mathcal{C})
$$

which can be shown to be an embedding. The closure of $\Theta\left(\mathcal{H}^{n}(\Gamma)\right)$ is compact and defines the Morgan-Shalen compactification, denoted by ${\overline{\mathcal{H}^{n}(\Gamma)}}^{M S}$.

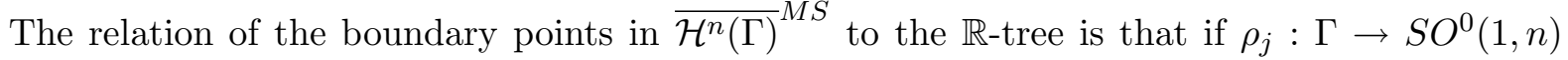
represent a unbounded sequence of points in $\mathcal{H}^{n}(\Gamma)$ which converges to a point $\left\{\ell_{\infty}(\gamma)\right\}_{\gamma \in \mathcal{C}} \in P(\mathcal{C})$, then there exists an $\mathbb{R}$-tree with a $\Gamma$-action whose translation length function projects to the point $\left\{\ell_{\infty}(\gamma)\right\}_{\gamma \in \mathcal{C}}$ in $P(\mathcal{C})$ (see [MS1] and also [Mor2] for a summary).

The construction in [MS1] is purely algebraic and related to compactifications of affine varieties via the relative growth rates of regular functions at infinity. On the other hand, a geometric way to see how $\mathbb{R}$-trees with $\Gamma$-action occur in the boundary of $\mathcal{H}^{n}(\Gamma)$ was given in [Bes2] [Pau1], which is summarized in [Kap, Chap. 10] [Ot2, Chap. 2]. Briefly, it can be described as follows.

1. Scale down $\mathbf{H}^{n}$ to get the tangent cone at infinity $T_{\infty} \mathbf{H}^{n}$, an $\mathbb{R}$-tree with a nontrivial $\Gamma$-action.

2. Take the $\Gamma$-invariant minimal $\mathbb{R}$-tree contained in $T_{\infty} \mathbf{H}^{n}$.

In the following, assume that $\Gamma$ is not an elementary discrete subgroup of the isometry group of $\mathbf{H}^{n}$. Let $g_{1}, \cdots, g_{m}$ be a set of generators of $\Gamma$.

Let $d(\cdot, \cdot)$ be the distance function on $\mathbf{H}^{n}$. Define a function $D_{j}(x)$ on $\mathbf{H}^{n}$ by

$$
D_{j}(x)=\max \left\{d\left(x, \rho_{j}\left(g_{1}\right)\right), \cdots, d\left(x, \rho_{j}\left(g_{m}\right)\right) .\right.
$$

Since the functions $d\left(x, \rho_{j}\left(g_{1}\right)\right), \cdots, d\left(x, \rho_{j}\left(g_{m}\right)\right)$ are convex and bounded from below (note that the $\Gamma$-action is proper), the minimum value of $D_{j}(x)$ is achieved (see [Kap, p. 240]). Let $x_{j} \in \mathbf{H}^{n}$ be such a minimum point. Since the isometry group of $\mathbf{H}^{n}$ acts transitively on $\mathbf{H}^{n}$, by conjugating $\rho_{j}$ by suitable elements, we can assume that $x_{j}$ is equal to a fixed basepoint $x_{0}$ in $\mathbf{H}^{n}$. 
In most cases we are interested in, $\Gamma$ is not virtually nilpotent, i.e., does not contain a nilpotent subgroup of finite index. Then the Margulis Lemma shows that $D_{j}$ is uniformly bounded away from zero. Since $\rho_{j}$ is not bounded and hence does not converge to a faithful discrete representation, $D_{j}$ is not bounded either. Assume that $D_{j} \rightarrow+\infty$. Scale the metric $d$ on $\mathbf{H}^{n}$ to $\frac{1}{D_{j}} d$ and take the limit of the sequence of pointed metric spaces $\left(\mathbf{H}^{n}, \frac{1}{D_{j}} d, x_{0}\right)$.

Proposition 4.2.3 As $D_{j} \rightarrow+\infty$, the sequence of pointed metric spaces $\left(\mathbf{H}^{n}, \frac{1}{D_{j}} d, x_{0}\right)$ converges to a geodesic metric space $\left(T_{\infty} \mathbf{H}^{n}, d_{\infty}, x_{0}\right)$ in the following sense: For any compact subset $K \ni x_{o}$ in $T_{\infty} \mathbf{H}^{n}$, there exists a compact subset $K_{j} \ni x_{0}$ in $\mathbf{H}^{n}$ such that $\left(K_{j}, \frac{1}{D_{j}} d\right)$ converges to $\left(K, d_{\infty}\right)$ in the sense of Gromov-Hausdorff. The metric space $T_{\infty} \mathbf{H}^{n}$ is called the tangent cone at infinity. It is an $\mathbb{R}$-tree and branches everywhere. In $T_{\infty} \mathbf{H}^{n}$, every point is the intersection of infinitely many distinct geodesics, where a geodesic is an isometric embedding of $\mathbb{R}$.

To understand the limit, we note that triangles in $\mathbf{H}^{n}$ with side lengths greater than $D_{j}$ with center $x_{0}$ in the metric $d$ converge under the scaling to a $Y$-shaped subset with three segments coming out of the point $x_{0}$. Changing these triangles and increasing their side lengths will produce infinitely many geodesics coming out of the point $x_{0}$. To see that other points on these rays are also branch points, we use large triangles centered at other points. Specifically, let $\gamma(t), t \in(-\infty,+\infty)$ be a geodesic with $\gamma(0)=x_{0}$. Fix any $t>0$. Let $x_{j}, y_{j}, z_{j}$ be the vertexes of a family of triangles whose side $\overline{x_{j} y_{j}}$ converges to the geodesic $\gamma$. Choose a point $w_{j}$ on the side $\overline{x_{j} y_{j}}$ such that the sequence $w_{j}$ converges to $\gamma(t)$. Then triangles in $\mathbf{H}^{n}$ of long sides and with center $w_{j}$ will converge to geodesics intersecting at $\gamma(t)$. This shows that the tangent cone at infinity $T_{\infty} \mathbf{H}^{n}$ is an $\mathbb{R}$-tree which branches infinitely many times at every point.

Remark 4.2.4 The existence of the tangent cone at infinity of $\mathbf{H}^{n}$ is a special case of the tangent cone at infinity of symmetric spaces in [KL2]. In fact, according to [KL2, §5, §2.4], one first chooses a non-principal ultrafilter, which is a finitely additive probability measure $\omega$ on the collection of subsets of the natural numbers $\mathbb{N}$ such that $(1) \omega(S)=0$ or 1 for any $S \subset \mathbb{N}$, and $(2) \omega(S)=0$ if $S$ is finite. Roughly, subsets $S$ with $\omega(S)=1$ are suitable subsequences of $\mathbb{N}$, and ultralimits are basically limits for some complicated procedure of taking limits over subsequences. It is known that any sequence of pointed metric spaces has an ultralimit associated with the ultrafilter $\omega$. In particular, every metric space $(M, d)$ has a tangent cone at infinity, defined as the ultralimit of $\left(M, \frac{1}{D_{j}} d, x_{0}\right)$ associated with $\omega$, where $D_{j}$ is a sequence going to infinity. For symmetric spaces, it is believed by many people that the tangent cone at infinity should not depend on the choice of the ultrafilter $\omega$, though there is no written proof of it.

Remark 4.2.5 The reason for the choice of the scaling constant $D_{j}$ is that for any element in $\Gamma$, its translation length in the limit $\mathbb{R}$-tree is finite, and for some elements, the translation length is not zero. For this purpose, the assumption that the minimum point $x_{j}$ is equal to (or conjugated to) the fixed basepoint $x_{0}$ is also important. Basically, $x_{0}$ is not far away from the axes of translation of the elements $g_{1}, \cdots, g_{m}$. Certainly we can modify $D_{j}$ by any positive number. This corresponds to the factor $\mathbb{R}^{\times}$in the projection map $\mathbb{R}^{\mathcal{C}} \rightarrow P(\mathcal{C})=\mathbb{R}^{\mathcal{C}} / \mathbb{R}^{\times}$above.

Consider $\left(\mathbf{H}^{n}, \frac{1}{D_{j}} d, x_{0}\right)$ as a pointed $\Gamma$-space through the representation $\rho_{j}$. After passing to a subsequence, it converges to the space $T_{\infty} \mathbf{H}^{n}$ with an isometric action of $\Gamma$. This finishes Step (1).

It should be emphasized that this $\mathbb{R}$-tree $T_{\infty} \mathbf{H}^{n}$ with the $\Gamma$-action is not necessarily the $\mathbb{R}$-tree which appears in the boundary of $\mathcal{H}^{n}(\Gamma)$. In fact, let $\mathcal{T}$ be the minimal invariant sub- $\mathbb{R}$-tree in $T_{\infty} \mathbf{H}^{n}$, where by a minimal $\Gamma$-tree, we mean that that it does not contain any proper $\Gamma$-invariant 
subtree. (see [Pau1] [Kap, Theorem 10.12, Theorem 10.24] [Ot2, pp. 28-32]). The translation lengths of the elements of $\Gamma$ on $\mathcal{T}$ are finite but not all zero and project to the limit of $\rho_{j}$ in $P(\mathcal{C})$. Then $\mathcal{T}$ is the $\mathbb{R}$-tree in the boundary of $\mathcal{H}^{n}(\Gamma)$ to which the sequence $\rho_{j}$ converges.

Remark 4.2.6 There are reasons for picking the minimal $\Gamma$-invariant subtree $\mathcal{T}$ instead of the whole tangent cone $T_{\infty} \mathbf{H}^{n}$. One reason is that minimal non-unipotent $\Gamma$-trees are uniquely determined by their translation length functions [CM] [Kap, Theorem 10.15]. Since the Morgan-Shalen compactification of $\mathcal{H}^{n}(\Gamma)$ is obtained by embedding into the projective space via the translation length function, it is natural to require that the boundary trees are minimal in order to get unique ones.

The $\mathbb{R}$-trees with $\Gamma$-action which appear in the boundary of $\mathcal{H}^{n}(\Gamma)$ satisfy the following important property [MS2, Theorem IV.1.2] (see also [Kap, Theorem 10.24] [Mor2, Lemma 6, Addendum $\left.\left.8^{\prime}\right]\right):$

Proposition 4.2.7 Let $T$ be such an $\mathbb{R}$-tree in the boundary of $\mathcal{H}^{n}(\Gamma)$. Then $\Gamma$ has no fixed point on $T$, and for every nondegenerate segment in $T$, its stabilizer in $\Gamma$ is small, i.e., virtually abelian.

Corollary 4.2.8 Suppose every action of $\Gamma$ on an $\mathbb{R}$-tree with small stabilizers of edges (nondegenerate segments) has a fixed point, then $\mathcal{H}^{n}(\Gamma)$ is compact.

The problem is to find conditions under which the $\Gamma$-action in the corollary has no fixed point. This is given as follows [MS2, Chap. IV] [Mor2, Theorem 12].

Proposition 4.2.9 Let $\Gamma$ be a finitely generated group isomorphic to the fundamental group of a 3-manifold. If $\Gamma$ has an action without fixed points on an $\mathbb{R}$-tree with small edge stabilizers, then $\Gamma$ splits as either (I) $\Gamma=A \star_{C} B$ with $C$ virtually abelian and $C \neq A, C \neq B$, or (II) $\Gamma=A \star_{C}$ with $C$ virtually abelian.

The compactness result of Thurston in Theorem 4.2.2 follows from this result and Corollary 4.2.8.

Remark 4.2.10 There are alternative approaches to issues related to the compactness of $\mathcal{H}^{n}(\Gamma)$. See $[\mathrm{BF}][\mathrm{GLP}][\mathrm{Ch}]$. Characterizations of certain nice actions of surface groups on $\mathbb{R}$-trees are given in [Sk] and used crucially in [Ot2]. For other applications of $\mathbb{R}$-trees to topology, see [KlS] [AS] [Wol1] [DDW].

\section{$4.3 \quad \mathbb{R}$-Euclidean buildings}

In $\S 4.1$, we recalled the definition of $\mathbb{R}$-trees. They are special cases of $\mathbb{R}$-Euclidean buildings, which will be recalled briefly.

As seen above, an $\mathbb{R}$-tree is not a 1-dimensional simplicial complex. It contains infinitely many geodesics, which correspond to apartments in rank-1 Euclidean buildings. In general $\mathbb{R}$-trees, geodesics are also called apartments, but they can not be decomposed into a locally finite disjoint union of chambers and chamber faces as in the case of simplicial trees.

Roughly, an $\mathbb{R}$-Euclidean building of rank $r \geq 1$ is a metric space which contains many compatible apartments, each of them is an affine (or Euclidean) space of dimension $n$ admitting the action of a generalized Weyl group.

More precisely, let $\bar{W}$ be a finite Coxeter group acting essentially on a vector space $\mathbb{R}^{r}$. Let $\mathbf{A}$ be the affine space associated with $\mathbb{R}^{r}$. Define $W$ to be the group of affine isometries of $\mathbf{A}$ whose 
vector part is $\bar{W}$, i.e., $W=\bar{W} \cdot \mathbb{R}^{r}$. We can also take a subgroup of $W$ such that its vector part is equal to $\bar{W}$ but the translation part is a dense subgroup of $\mathbb{R}^{r}$. Using reflections in $W$, we can define half-apartments, sectors and sector faces as in the case of Euclidean reflection complexes. (It should be emphasized that we could not define chambers since the walls, i.e., the faces of half apartments, are dense in the apartments).

Then an $\mathbb{R}$-Euclidean building modeled on $(\mathbf{A}, W)$ is a metric space $\Delta$ together with a family $\mathcal{F}$ of isometric embeddings of $\mathbf{A}$ into $\Delta$, which are called the apartments in $\Delta$, satisfying the following conditions:

1. If $w \in W$ and $f \in \mathcal{F}$, then $f \circ w \in \mathcal{F}$.

2. If $f, f^{\prime} \in \mathcal{F}$, then $S=f^{-1}\left(f^{\prime}(\mathbf{A})\right)$ is closed and convex in $\mathbf{A}$ (this should be interpreted as the intersection of the two apartments $f(\mathbf{A})$ and $f^{\prime}(\mathbf{A})$ ), and there exists $w \in W$ such that $\left.f\right|_{S}=\left.f^{\prime} \circ w\right|_{S}$.

3. Any two points in $\Delta$ lie in a common apartment.

4. Any two sectors contain subsectors which are contained in a common apartment.

5. If $A_{1}, A_{2}, A_{3}$ are three apartments such that each of $A_{1} \cap A_{2}, A_{1} \cap A_{3}$ and $A_{2} \cap A_{3}$ is a half-apartment, then $A_{1} \cap A_{2} \cap A_{3} \neq \emptyset$.

Remark 4.3.1 We basically follow the definition given in [Ro3, Appendix 3]. Conditions (1)-(4) are reasonable and clearly expected, and (5) can be replaced by the following: For any apartment $f(\mathbf{A})$ and a point $p$ in it, there exists a retraction $\rho: \Delta \rightarrow f(\mathbf{A})$ such that $\rho^{-1}(p)=p$, and the restriction to each apartment is distance decreasing.

Remark 4.3.2 There are other definitions of $\mathbb{R}$-Euclidean buildings or affine $\mathbb{R}$-buildings (and more generally affine $\Lambda$-buildings). The first non-discrete buildings were introduced by Bruhat and Tits in [BT1, §7]. The general axioms of non-discrete buildings were first introduced by Tits in [Ti7]. In [KL2, 4.1.2], R-Euclidean buildings were defined in a slightly different way. In [Par] Parreau showed that they are equivalent. See also the survey $[\mathrm{KT}]$.

As recalled earlier in $\S 3.2$, for any field $F$ with a non-discrete valuation $\nu: F^{\times} \rightarrow \mathbb{R}_{\geq 0}$, there is a natural $\mathbb{R}$-tree associated with $S L(2, F)$. In $[\mathrm{BT} 1, \S 7.4]$, for a linear connected and simply connected semisimple algebraic group $\mathbf{G}$ defined over such a field $F$, there is an $\mathbb{R}$-Euclidean building $\Delta^{B T}(\mathbf{G})$. When the valuation is discrete, the building is a simplicial complex and can be described by either specifying the set of simplexes, for example, the vertexes and chambers; or using a $B N$-pair. In the case of rank 1, i.e., $\Lambda$-trees, we specified the set of vertexes and described the edges using a $\Lambda$-distance. This approach seems to be difficult to generalize in the higher rank case. Hence, Bruhat and Tits $[\mathrm{BT} 1, \S 7]$ used another construction which also gives an alternative construction of the usual Euclidean buildings.

To motivate this construction, we consider the Bruhat-Tits building $\Delta^{B T}(\mathbf{G})$ of a simply connected algebraic group over a local field $k_{\mathfrak{p}}$. Then $\mathbf{G}\left(k_{\mathfrak{p}}\right)$ acts transitively on the set of apartments. Fix an apartment $\Sigma$, which can be identified with an affine space $\mathbf{A}$. Then there is a surjective map

$$
\mathbf{G}\left(k_{\mathfrak{p}}\right) \times \Sigma \rightarrow \Delta^{B T}(\mathbf{G}) .
$$

The fibers of this map can be described explicitly in terms of parabolic subgroups.

The construction for the non-discrete case is a generalization of this (see [Ti3, §2] and also [Lan2]). Let $\mathbf{T}$ be a maximal split torus in $\mathbf{G}$, and $N$ the normalizer of $\mathbf{T}(F)$ in $\mathbf{G}(F)$. Then the 
group $X_{*}(\mathbf{T})=\operatorname{Hom}_{F}\left(G L_{1}, \mathbf{T}\right)$ determines a real vector space $V=X_{*}(\mathbf{T}) \otimes \mathbb{R}$. Let $Z=Z(\mathbf{T}(F))$ be the centralizer of $\mathbf{T}(F)$ in $\mathbf{G}(F)$. There is a natural homorphism $\nu: Z \rightarrow V$. Denote its kernel by $Z_{c}$. Then $\Lambda=Z / Z_{c}$ is a free abelian group, and $W=N / Z_{c}$ is an extension of a finite Coxeter group by $\Lambda$, and acts isometrically on the affine space $\mathbf{A}$ associated with $V$ (see [Ti3, $\S 1.2]$ ).

In the affine space $\mathbf{A}$, we can define various subgroups associated to subsets, in particular to points, for example, $\hat{P}_{x}, x \in \mathbf{A}[\mathrm{BT} 1, \S 7.1]$. Then the associated building $\Delta^{B T}(\mathbf{G})$ is defined as a quotient of $\mathbf{G}(F) \times \mathbf{A}$ under the following equivalence relation: Two pairs $(g, x)$ and $(h, y)$ are equivalent if there exists $n \in N$ such that

$$
y=n \cdot x, \quad \text { and } g^{-1} h n \in \hat{P}_{x} .
$$

\subsection{Quasi-isometries and tangent spaces at infinity of symmetric spaces}

As seen in the proof of Mostow strong rigidity in Theorem 2.8.1, quasi-isometries play an important role. In fact, a crucial step is that under the equivariant quasi-isometry $\varphi$ in Equation 2.8.2, the image of a flat $F$ in $X$ lies within a bounded neighborhood of a flat $F^{\prime}$ in $X^{\prime}$ (Proposition 2.8.4).

Gromov initiated a systematic study of global geometry of groups in terms quasi-isometries (see $[\mathrm{Gr}]$ ). One problem concerns the rigidity of symmetric spaces of higher rank under quasi-isometries, a conjecture due to Margulis in late 1970's, which was proved by Kleiner and Leeb [KL2, Theorem 1.1.3] and later Eskin and Farb [EF1]. One result in the proof says that in the above result of Mostow in Proposition 2.8.4, the equivariance condition on the quasi-isometry can be removed [KL2, Corollary 7.1.5].

Proposition 4.4.1 Let $X, X^{\prime}$ be two symmetric spaces of noncompact type without rank one irreducible factors. If they are quasi-isometric, then they are isometric after the metrics on the irreducible factors are suitably scaled, and any quasi-isometry between them is within a bounded distance of an isometry.

Remark 4.4.2 See [KL1] [Esk] [EF1] [Scr1] [Scr2] [FS] and the review of [Esk] by L.Mosher for a summary of other results related to classifications of lattices in semisimple Lie groups up to quasiisometry. Parts of the proofs in [Esk] follow the proof of the Mostow strong rigidity and use the spherical Tits buildings in a substantial way.

The results in [KL2, Theorem 1.1.3] are more general than stated here. The proof in [KL2] of this result goes as follows:

1. Choose an ultrafilter $\omega$ and obtain the tangent cones at infinity $T_{\infty} X$ and $T_{\infty} X^{\prime}$.

2. Show that $T_{\infty} X$ and $T_{\infty} X^{\prime}$ are $\mathbb{R}$-Euclidean buildings of rank at least two, and a quasiisometry $\varphi: X \rightarrow X^{\prime}$ induces a homeomorphism between the two $\mathbb{R}$-Euclidean buildings.

3. The homeomorphism maps an apartment in $T_{\infty} X$ to an apartment in $T_{\infty} X^{\prime}$.

4. Let $F$ be a flat in $X$. Then $\varphi(F)$ lies in a bounded distance of a flat $F^{\prime}$ of $X^{\prime}$.

5. Follow similar steps in the proof of Mostow strong rigidity to prove the result.

For a more detailed outline and summary of the proof, see [KL2, §1.2].

Remark 4.4.3 In the proof of the Mostow strong rigidity, we push things out to infinity to get an isomorphism of the spherical Tits buildings. In Step (1) of the proof in [KL2] outlined here, we pull in infinity towards a basepoint to get the tangent cones at infinity, and the symmetric spaces become $\mathbb{R}$-Euclidean buildings. Both have the common feature that they ignore finite ambiguities and turn a quasi-isometry into more precise maps. 


\section{Other applications of buildings}

In $\S \S 2-4$, we have discussed applications of buildings to many problems in (differential) geometry and topology. In this last section, we indicate very briefly other applications.

\subsection{Applications in algebraic geometry}

Algebraic groups and their parabolic subgroups occur naturally in geometric invariant theory and torus embeddings (or toric varieties) and toroidal compactifications of Hermitian locally symmetric spaces. Because of this, spherical Tits buildings have played an important role in these subjects.

Given a reductive algebraic group $\mathbf{G}$ and a variety $X$ where $\mathbf{G}$-acts, a basic problem in geometric invariant theory is to find geometric quotients of suitable open subsets $U$ of $X$ which are invariant under $\mathbf{G}$ and the geometric quotient of $U$ by $\mathbf{G}$ exists. These open subsets $U$ can be defined in terms of notions of stability and semi-stability of points [Mu3, Chap. I, §4]. Now these stability conditions are related to the positivity of certain numerical invariants of 1-parameter subgroups $\lambda$ in $\mathbf{G}$ [Mu3, Chap. II, $\S 1$, Theorem 2.1]. Considerations of these invariants naturally lead to parabolic subgroups $\mathbf{P}(\lambda)$ of $\lambda$ [Mu3, Chap. II, $\S 2]$. This connection with parabolic subgroups gives an equivalence relation on the set of 1-parameter subgroups of $\mathbf{G}$, which leads to a rational flag complex [Mu3, Chap. II, Definition 2.5]. The rational flag complex is contained in the spherical Tits building of $\mathbf{G}$ as a dense subset and its completion gives the spherical Tits building $\Delta(\mathbf{G})$ [Mu3, Chap. II, pp. 59-61]. This embedding in the building $\Delta(\mathbf{G})$ allows one to define semi-convex subsets and convex subsets of of the rational flag complex of 1-parameter subgroups [Mu3, Chap. II, Definition 2.10], which are important in questions about stability [Mu3, Chap. II, §3].

The 1-parameter subgroups $\lambda$ of $\mathbf{G}$ in [Mu3] describe the geometry at infinity of $\mathbf{G}$. A more explicit relation between the structure at infinity of $\mathbf{G}$ and the spherical Tits building $\Delta(\mathbf{G})$ is given in [KKMS, Chap. IV, §2]. In fact, a toroidal embedding (or a partial compactification) of a semisimple linear algebraic group $\mathbf{G}$ over an algebraically closed field $k$ was constructed. The boundary is decomposed into boundary components whose normalizers are exactly the parabolic subgroups of $\mathbf{G}$. The inclusion relations between the closures of the boundary components define a complex which turns out to be exactly the spherical Tits building $\Delta(\mathbf{G})$. One reason is that the polyhedal cone decomposition for the torus embeddings involved is exactly the Weyl chamber decomposition [KKMS, p. 185, p. 187].

Simplicial complexes constructed from only a proper subset of the set of all parabolic subgroups are also important in toroidal compactifications of Hermitian locally symmetric spaces [Mu1] [AMRT] [HKW] [HZ1] [HZ2]. In fact, the theories in [KKMS] were used in the sequel [AMRT] to explicitly resolve the singularities of the Baily-Borel compactification [BB] of Hermitian locally symmetric spaces, or quotients of bounded symmetric domains by arithmetic quotients. The resolutions are given by the toroidal compactifications. The reason why the Tits buildings or related complexes come in is the same basically as before. The normalizers of boundary components of (partial) compactifications of groups and bounded symmetric domains are often certain parabolic subgroups, and the inclusion relation between their closures lead to simplicial complexes which are often closely related to spherical Tits buildings. For example, in the partial Baily-Borel compactification of a bounded symmetric domain, only maximal parabolic subgroups arise as the normalizer of the boundary components. See $[\mathrm{HKW}]$ for detailed discussions about the (partial) Tits buildings and relations to the Baily-Borel compactification, and the toroidal compactifications of the moduli space of abelian surfaces. The discussions here are related to the point of view in $\S 2.12$.

The spherical Tits buildings of dimension 1 are crucial in the important paper [Mu2] on uniformization of curves over complete local rings by Schottky groups (see $[\mathrm{FvP}]$ for more recent results 
and applications and also [GvP] [vP], where trees and currents on trees were used), and the generalization in [Mus]. Related results about Arakelov intersection of arithmetic varieties are given in [Man], which motivated [Wer3], where the Bruhat-Tits building of $P G L(n)$ was used to compute certain Arakelov intersection. As pointed out earlier, Euclidean buildings of dimension 1 are trees. Certain trees, called valuation trees in [FJ], are important in describing the singularities in complex analysis and algebraic geometry.

The theory of Bruhat-Tits buildings plays an important role in [Ra3] on work related to a conjecture by Grothendieck and Serre that given any principal bundle over a smooth connected $k$-scheme with fiber given by a smooth reductive group scheme over $k$, if it is trivial over some nonempty open subset of $X$, then it is locally trivial in the Zariski topology. The Bruhat-Tits theory is also used in a new proof in $[\mathrm{GaR}]$ of some results of Bott on the loop space of a compact, simply connected, simple Lie group and hence of the Bott periodicity for the unitary group.

Buildings also occur naturally in rigid analytic geometry over local non-Archimedean fields. An important rigid analytic space is the p-adic symmetric space $\mathcal{X}_{n}$ associated with the group $P G L(n, K)$, introduced by Drinfeld in [Dr] (see also [BoC] and [vPV]). There is a well-defined surjective equivariant map from $\mathcal{X}_{n}$ to the Bruhat-Tits building of $P G L(n, K)$, called the reduction map [Te]. This reduction map plays an important role in computing the cohomology of $\mathcal{X}_{n}$ and its quotients by cocompact discrete groups in [SS2] (see also [Te]).

A natural boundary of $\mathcal{X}_{n}$ is the topological spherical Tits building of $P G L(n, K)$. As recalled in $\S 3.5$, this topological Tits building appears naturally as the boundary of the Bruhat-Tits building of $P G L(n, K)$. An important property of this boundary of $\mathcal{X}_{n}$ is that the reduction map extends to an equivariant map to the boundaries. This plays an important role in [ST], which generalizes the Poisson integral transformation of Riemannian symmetric spaces.

Buildings are also related to p-adic analytic spaces in the sense of [Ber2]. In fact, rigid analytic spaces are analog over local non-Archimedean fields of complex analytic spaces but do not have the corresponding topological properties, for example, local compactness and local arcwise connectedness. To overcome this problem, p-adic analytic spaces were introduced in [Ber2]. Roughly, to a rigid analytic space $X$, there is a p-adic analytic space $X^{a n}$, which is a kind of completion (or filling in holes) in a suitable way. Under suitable assumptions on $X$, the underlying topological space of $X^{a n}$ is locally connected, locally compact and locally arcwise connected. There is a canonical surjective morphism $X^{\text {an }} \rightarrow X$ [Ber2, Theorem 3.4.1].

Buildings and trees are related to these spaces in several ways. In fact, one dimensional quasipolyhedrons were introduced in [Ber2, Definition 4.1]. The simply connected ones are certain trees. If $X$ is a smooth geometrically connected projective curve of genus $g \geq 1$, then $X^{a n}$ is a quasipolyhedron whose Betti number is at most $g$ [Ber2, Theorem 4.3.2]. When $\mathbf{G}$ is a semisimple Chevalley group over a local non-Archimedean field, then its Bruhat-Tits building $\Delta^{B T}(\mathbf{G})$ can be embedded into $\mathbf{G}^{a n}$ in two ways [Ber2, Theorems 5.4.2 and 5.4.4]. This is important in understanding the homotopy type of some p-adic analytic spaces [Ber2, Chap. 6]. When $\mathbf{P}$ is a parabolic subgroup of $\mathbf{G}$, then the Bruhat-Tits building $\Delta^{B T}(\mathbf{G})$ can also be embedded into $\mathbf{G}^{a n} / \mathbf{P}^{a n}$. Since the latter is compact, the closure of $\Delta^{B T}(\mathbf{G})$ under this embedding gives a compactification of $\Delta^{B T}(\mathbf{G})$ [Ber2, Remarks 5.5.2].

The results mentioned above are related to the reductive group $P G L(n, K)$. See [RaZ] for generalizations of some of these results to some other reductive groups over local non-Archimedean fields. 


\subsection{Applications in representation theory}

For semisimple algebraic groups over local fields, construction of the Bruhat-Tits buildings is only part of the Bruhat-Tits theory, which describes in detail various subgroups and decompositions. In fact, the geometry of the Bruhat-Tits buildings allow one to construct various filtrations and subgroups, which are fundamental to the representation theory of $p$-adic groups. The basic point is that the geometry of the Bruhat-Tits buildings can be used to parametrize various objects. See [Yu2] for a survey and introduction to the Bruhat-Tits theory and [MP1] [MP2] [SS1] [SS2] [ST] [De1] [De2] [Moy] [KM] [PYu] [Te] for many applications in representation theory of $p$-adic groups.

Another important application of the Bruhat-Tits theory concerns topological central extensions of semisimple groups over local fields [PrR1] [PrR2], which motivated the Moy-Prasad filtration in [MP1], which in turn has become a basic tool in representation theory and related subjects.

The Bruhat-Tits theory is also important in computing the volume of $S$-arithmetic quotients of semisimple algebraic groups [Pr1].

\subsection{Random walks and the Martin boundary}

Random walks on graphs and in particular on trees are natural problems. They are related to harmonic functions, which are functions on the set of vertexes and satisfy the condition that the value at any point is equal to the average (or weighted average) of values over nearby points. Closely related problems are to determine the Poisson boundary and the Martin compactification. These problems have been solved for trees [An] [Woe].

For Euclidean buildings of rank at least 2, we can also define functions on the set of vertexes, or the set of simplexes of any fixed dimension, and harmonic functions. The related problems on the Poisson boundary and the Martin compactification have been only partially understood. See $[\mathrm{CW}],[\mathrm{Ge}]$, and the papers and references in $[\mathrm{Ko}]$.

\subsection{Finite groups, finite geometry and algebraic $K$-groups}

Spherical Tits buildings or rather $B N$-pairs have played an important role in understanding finite groups and their representations [Car1] [Car2] [So]. See [Su1] [Su2] [Su3] [Har] for characterization and classification of finite simple groups of Lie type.

Buildings are also important in incidence geometry (see the many articles in [Bue]), finite geometry (see [Hir] [Tha2] and the references there) and coding theory [MS].

An important conjecture of Bass in algebraic K-theory concerns finite generation of $K$-groups associated with a commutative ring which is finitely generated as an algebra over $\mathbb{Z}$ (see [FW, p. 53] for the precise statement). This conjecture has been proved by Quillen for finite fields [Qu2], the ring of integers of any number field [Qu1] and the ring of regular functions of a smooth affine curve over a finite field (written up by Grayson in [Gra3]. See also [Ro2, §13]).

In $[\mathrm{Qu}]$, the Solomon-Tits theorem that the homotopy type of a spherical Tits building is a bouquet of spheres was used crucially. In [Gra3], Bruhat-Tits buildings of $G L(n)$ over nonarchimedean local fields induced from function fields played an important role.

As pointed out in [Qu1], the finiteness result also holds for the ring of $S$-integers $\mathcal{O}_{S}$ in any number field, by combining the results for finite fields and a localization result. The result in [Gra3] can also be restated as that the $K$-groups of rings of $S$-integers (see $\S 3.6$ for definition) of the function field of a smooth projective curve over a finite field are finitely generated. 


\subsection{Hyperbolic buildings and combinatorial group theory}

It is well-known that simplicial trees are fundamental in combinatorial group theory (see [Se2]). For a survey of more recent developments and generalizations, see the book [BL] and [BuM1] [BuM2]. Many natural discrete subgroups in the automorphism group of trees have been constructed from Kac-Moody groups and subgroups. In these studies, the buildings associated to the infinite dimensional Kac-Moody groups (or algebras) are used. As pointed out in the introduction, many such buildings are hyperbolic, i.e., the apartments have negative curvature. This seems to be an active ongoing research area. See [CG1] [CG2] [Re1] [Re4] [DJ] [Ja] [Laf1] [Laf2].

Buildings are also used by Deligne in [Del] to compute the homology groups of hyperplane arrangements.

\subsection{Algebraic combinatorics}

Complexes similar to Coxeter complexes and spherical Tits buildings also occur naturally in algebraic combinatorics. In fact, they are very important special examples of these general complexes.

In $[\mathrm{Bj}]$, it is shown that Coxeter complexes and Tits buildings are shellable and Cohen-Macaulay complexes. A generalization of Coxeter complexes for a group with a minimal set of generators is given in $[\mathrm{BaR}]$. Such complexes and their homology give useful representations of the group.

Combinatorial flag varieties were introduced in [BGW] using suitable partial ordering on matroids, and their shellability and other applications are studied in [BGS]. Semibuildings were introduced in [Her]. Other results related to shellability are given in [ER] [Was].

Relations between groups and abstract regular polytopes are explained in [McS]. For generalized polygons, see [Tha1].

\subsection{Expanders and Ramanujan graphs}

Expanders have been used to construct efficient networks. An important class of expanders consists of the class of Ramanujan graphs. Bruhat-Tits buildings of dimension 1 have played an important role in studying Ramanujan graphs. Higher dimensional Bruhat-Tits buildings have also been used to construct hypergraphs.

For a general introduction to Ramanujan graphs and connections to modular forms, see the book [Li2], surveys [Li3] [Li4]. For constructions of Ramanujan graphs, see also [LPS] [Mors]; for constructions of Ramanujan hypergraphs or hypercomplexes, see [Li1] [LSV1] [LSV2] [CSZ] [Sar]. See also [ChL1] [ChL2] for applications of modular forms to Ramanujan graphs.

\section{References}

[Abe1] H.Abels, Finiteness properties of certain arithmetic groups in the function field case, Israel J. Math. 76 (1991) 113-128.

[Abe2] H.Abels, Finite presentability of S-arithmetic groups. Compact presentability of solvable groups, Lecture Notes in Mathematics, vol. 1261, Springer-Verlag, 1987. vi+178 pp.

[AbA] H.Abels, P.Abramenko, On the homotopy type of subcomplexes of Tits buildings, Adv. Math. 101 (1993) 78-86.

[Abr1] P.Abramenko, Twin buildings and applications to S-arithmetic groups, Lecture Notes in Mathematics, vol. 1641, Springer-Verlag, 1996. x+123 pp. 
[Ab2] P.Abramenko, Finiteness properties of Chevalley groups over $F_{q}[t]$, Israel J. Math. 87 (1994) 203-223.

[AbN] P.Abramenko, G.Nebe, Lattice chain models for affine buildings of classical type, Math. Ann. 322 (2002) 537-562.

[AS] S.Adams, R.Spatzier, Kazhdan groups, cocycles and trees, Amer. J. Math. 112 (1990) 271-287.

[AB] R.Alperin, H.Bass, Length functions of group actions on $\Lambda$-trees, in Combinatorial group theory and topology, pp. 265-378, Ann. of Math. Stud., 111, Princeton Univ. Press, 1987.

[AM] R.Alperin, K.Moss, Complete trees for groups with a real-valued length function, J. London Math. Soc. 31 (1985) 55-68.

[An] A.Ancona, Thiorie du potentiel sur les graphes et les variitis, pp. 1-112, Lecture Notes in Math., 1427, Springer-Verlag, 1990.

[AF] C.Aravinda, F.Farrell, Exotic Negatively Curved Structures on Cayley Hyperbolic Manifolds, J. Differential Geom. 63 (2003) 41-62.

[AMRT] A.Ash, D.Mumford, M.Rapoport, Y.Tai, Smooth compactification of locally symmetric varieties, Math. Sci. Press, 1975. iv+335 pp.

[Bae] J.Baez, The octonions, Bull. Amer. Math. Soc. 39 (2001) 145-205.

[BB] W.Baily, A.Borel, Compactification of arithmetic quotients of bounded symmetric domains, Ann. of Math. 84 (1966) 442-528.

[BL1] A.Balser, A.Lytchak, Building-like spaces, preprint.

[BL2] A.Balser, A.Lytchak, Centers of convex subsets of buildings, preprint.

[Bal1] W.Ballmann, Lectures on spaces of nonpositive curvature, Birkhäuser Verlag, 1995.

[Bal2] W.Ballmann, Manifolds of nonpositive sectional curvature and manifolds without conjugate points, in Proceedings of the International Congress of Mathematicians, (Berkeley, Calif., 1986) pp. 484-490, Amer. Math. Soc., 1987.

[Bal3] W.Ballmann, Nonpositively curved manifolds of higher rank, Ann. of Math. 122 (1985) 597-609.

[BBE] W.Ballmann, M.Brin, P.Eberlein, Structure of manifolds of nonpositive curvature. I, Ann. of Math. 122 (1985) 171-203.

[BBS] W.Ballmann, M.Brin, R.Spatzier, Structure of manifolds of nonpositive curvature. II, Ann. of Math. 122 (1985) 205-235.

[BMS] W.Ballmann, M.Gromov, V.Schroeder, Manifolds of nonpositive curvature, in Progress in Mathematics, vol. 61, Birkhäuser Boston, 1985.

[BaR] E.Babson, V.Reiner, Coxeter-like complexes, Discrete Math. and Theoretical computer science 6 (2004) 223-251. 
[BL] H.Bass, A.Lubotzky, Tree lattices, in Progress in Mathematics, vol. 176, Birkhäuser, 2001. xiv +233 pp.

[Beh1] H.Behr, Higher finiteness properties of S-arithmetic groups in the function field case I, in Groups: topological, combinatorial and arithmetic aspects, pp. 27-42, London Math. Soc. Lecture Note Ser., 311, Cambridge Univ. Press, 2004.

[Beh2] H.Behr, Arithmetic groups over function fields. I. A complete characterization of finitely generated and finitely presented arithmetic subgroups of reductive algebraic groups, J. Reine Angew. Math. 495 (1998) 79-118.

[Ber1] V.Berkovich, The automorphism group of the Drinfeld half-plane, C. R. Acad. Sci. Paris Sir. I Math. 321 (1995) 1127-1132.

[Ber2] V.Berkovich, Spectral theory and analytic geometry over non-Archimedean fields, Mathematical Surveys and Monographs, vol. 33, American Mathematical Society, 1990. x+169 pp.

[Ber3] V.Berkovich, p-adic analytic spaces, in Proceedings of the International Congress of Mathematicians, Vol. II (Berlin, 1998), Doc. Math. 1998, Extra Vol. II, 141-151.

[BCG] G.Besson, G.Courtois, S.Gallot, Entropies et rigiditis des espaces localement symitriques de courbure strictement nigative, Geom. Funct. Anal. 5 (1995) 731-799.

[Bes1] M.Bestvina, $\mathbb{R}$-trees in topology, geometry, and group theory, in Handbook of geometric topology, pp. 55-91, North-Holland, 2002.

[Bes2] M.Bestvina, Degenerations of the hyperbolic space, Duke Math. J. 56 (1988) 143-161.

[BF] M.Bestvina, M.Feighn, Stable actions of groups on real trees, Invent. Math. 121 (1995) 287-321.

[Bj] A.Björner, Some combinatorial and algebraic properties of Coxeter complexes and Tits buildings, Adv. in Math. 52 (1984) 173-212.

[BoC] J.Boutot, H.Carayol, Uniformisation p-adique des courbes de Shimura: les théorèmes de Čerednik et de Drinfeld, in Courbes modulaires et courbes de Shimura, Astérique No. 196197, (1991), 7, 45-158 (1992).

[BJ1] A.Borel, L.Ji, Compactifications of symmetric and locally symmetric spaces, a volume in the series Mathematics: Theory and Applications by Birkhäuser, to be published in 2005.

[BJ2] A.Borel, L.Ji, Lectures on compactifications of symmetric and locally symmetric spaces, Lie Theory, ed. by J.P.Anker, B.Orsted, in Progress in Mathematics, vol. 229, Birkhäuser, 2005, pp. 69-139.

[BoS1] A.Borel, J.P.Serre, Cohomologie d'immeubles et de groupes S-arithmitiques, Topology 15 (1976) 211-232.

[BoS2] A.Borel, J.P.Serre, Corners and arithmetic groups, Comment. Math. Helv. 48 (1973) 436491.

[BGS] A.Borovik, I.M.Gelfand, D.Stone, On the topology of the combinatorial flag varieties, Discrete \& computational geometry 27 (2002) 195-214. 
[BGW] A.Borovik, I.M.Gelfand, N.White, Combinatorial flag varieties, Journal of combinatorial theory, Series A 91 (2000) 111-136.

[BP1] M.Bourdon, H.Pajot, Quasi-conformal geometry and hyperbolic geometry, in Rigidity in dynamics and geometry, pp. 1-17, Springer, 2002.

[BP2] M.Bourdon, H.Pajot, Rigidity of quasi-isometries for some hyperbolic buildings, Comment. Math. Helv. 75 (2000) 701-736.

[BS] M.Bridson, G.Swarup, On Hausdorff-Gromov convergence and a theorem of Paulin, Enseign. Math. 40 (1994) 267-289.

[BH] M.Bridson, A.Haefliger, Metric spaces of non-positive curvature, Springer-Verlag, 1999.

[Br1] K.Brown, Buildings, Springer-Verlag, New York, 1989.

[Br2] K.Brown, Five lectures on buildings, in Group theory from a geometrical viewpoint (Trieste, 1990), pp. 254-295, World Sci. Publishing, 1991.

[Br3] K.Brown, Cohomology of groups, Graduate Texts in Mathematics, vol. 87, Springer-Verlag, New York, 1994.

[BrD] K.Brown, P.Diaconis, Random walks and hyperplane arrangements, Ann. Probab. 26 (1998) 1813-1854.

[BT1] F.Bruhat, J.Tits, Groupes réductifs sur un corps local, Inst. Hautes Etudes Sci. Publ. Math. No. 41 (1972) 5-251.

[BT2] F.Bruhat, J.Tits, Groupes riductifs sur un corps local. II. Schimas en groupes. Existence d'une donnie radicielle valuie, Inst. Hautes Itudes Sci. Publ. Math. No. 60 (1984) pp. 197-376.

[BT3] F.Bruhat, J.Tits, Schimas en groupes et immeubles des groupes classiques sur un corps local. II. Groupes unitaires, Bull. Soc. Math. France 115 (1987) 141-195.

[BT4] F.Bruhat, J.Tits, Schimas en groupes et immeubles des groupes classiques sur un corps local, Bull. Soc. Math. France 112 (1984) 259-301.

[Bue] F.Buekenhout, Handbook of incidence geometry. Buildings and foundations, NorthHolland, 1995.

[BuMo] M.Burger, N.Monod, Continuous bounded cohomology and applications to rigidity theory, Geom. Funct. Anal. 12 (2002) 219-280.

[BuM1] M.Burger, S.Mozes, Lattices in product of trees, Inst. Hautes Itudes Sci. Publ. Math. No. 92 (2000) 151-194.

[BuM2] M.Burger, S.Mozes, Groups acting on trees: from local to global structure, Inst. Hautes Itudes Sci. Publ. Math. No. 92 (2000) 113-150.

[BuS1] K.Burns, R.Spatzier, Manifolds of nonpositive curvature and their buildings, Inst. Hautes Itudes Sci. Publ. Math. 65 (1987) 35-59. 
[BuS2] K.Burns, R.Spatzier, On topological Tits buildings and their classification, Inst. Hautes Itudes Sci. Publ. Math. 65 (1987) 5-34.

[Bux] K.Bux, Finiteness properties of soluble S-arithmetic groups: a survey, in Groups: topological, combinatorial and arithmetic aspects, pp. 64-92, London Math. Soc. Lecture Note Ser., 311, Cambridge Univ. Press, 2004.

[Cal] E.Calabi, On compact, Riemannian manifolds with constant curvature. I, Proc. Sympos. Pure Math., Vol. III pp. 155-180, Amer. Math. Soc., 1961.

[CG1] L.Carbone, H.Garland, Lattices in Kac-Moody groups, Math. Res. Lett. 6 (1999) 439-447.

[CG2] L.Carbone, H.Garland, Existence of lattices in Kac-Moody groups over finite fields, Commun. Contemp. Math. 5 (2003) 813-867.

[CSZ] D.Cartwright, P.Solé, A.Zuk, Ramanujan geometries of type $\tilde{A}_{n}$, Discrete Math. 269 (2003) $35-43$.

[CW] D.Cartwright, W.Woess, Isotropic random walks in a building of type $C_{d}$, Math. Z. 247 (2004) 101-135.

[Cas] W.Casselman, On a p-adic vanishing theorem of Garland, Bull. Amer. Math. Soc. 80 (1974) 1001-1004.

[CV] E.Calabi, E.Vesentini, On compact, locally symmetric Kdhler manifolds, Ann. of Math. 71 (1960) 472-507.

[CT] J.Carlson, D.Toledo, Rigidity of harmonic maps of maximum rank, J. Geom. Anal. 3 (1993) 99-140.

[CP] G.Carlsson, E.Pedersen, Controlled algebra and the Novikov conjectures for $K$ - and $L$ theory, Topology 34 (1995) 731-758.

[Car1] R.Carter, Finite groups of Lie type. Conjugacy classes and complex characters, John Wiley \& Sons, 1985, xii+544 pp.

[Car2] R.Carter, Simple groups of Lie type, in Pure and Applied Mathematics, Vol. 28, 1972, viii+331 pp.

[ChL1] C.Chai, W.Li, Character sums, automorphic forms, equidistribution, and Ramanujan graphs, Part I. The Kloosterman sum conjecture over function fields, Forum Math 15 (2003) 679-699.

[ChL2] C.Chai, W.Li, Character sums, automorphic forms, equidistribution, and Ramanujan graphs, Part II. Eigenvalues of Terras graphs, Forum Math. 16 (2004) 631-661.

[CL] R.Charney, A.Lytchak, A metric characterization of spherical and Euclidean buildings, Geometry and Topology, Vol.5 (2001) 521-550.

[Ch] I.Chiswell, Nonstandard analysis and the Morgan-Shalen compactification, Quart. J. Math. Oxford Ser. (2) 42 (1991) 257-270.

[Co] K.Corlette, Archimedean superrigidity and hyperbolic geometry, Ann. of Math. 135 (1992) 165-182. 
[CM] M.Culler, J.Morgan, Group actions on R-trees, Proc. London Math. Soc. 55 (1987) 571604.

[DDW] G.Daskalopoulos, S.Dostoglou, R.Wentworth, On the Morgan-Shalen compactification of the $\mathrm{SL}(2, C)$ character varieties of surface groups, Duke Math. J. 101 (2000) 189-207.

[De1] S.DeBacker, Parametrizing nilpotent orbits via Bruhat-Tits theory, Ann. of Math. 156 (2002) 295-332.

[De2] S.DeBacker, Some applications of Bruhat-Tits theory to harmonic analysis on a reductive p-adic group, Michigan Math. J. 50 (2002) 241-261.

[Del] P.Deligne, Les immeubles des groupes de tresses giniralisis, Invent. Math. 17 (1972) 273302.

[Dr] V.Drinfeld, Coverings of p-adic symmetric domains, Funct. Anal. Appl. 10 (1976) 107-115.

[DJ] J.Dymara, T.Januszkiewicz Cohomology of buildings and their automorphism groups, Invent. Math. 150 (2002) 579-627.

[Eb1] P.Eberlein, Geometry of nonpositively curved manifolds, in Chicago Lectures in Mathematics, University of Chicago Press, 1996.

[Eb2] P.Eberlein, Isometry groups of simply connected manifolds of nonpositive curvature. II, Acta Math. 149 (1982) 41-69.

[ER] P.Edelman, V.Reiner, h-shellings and h-complexes, Adv. Math. 106 (1994) 36-64.

[Esc1] J.Eschenburg, Rank rigidity and symmetry, Irish Math. Soc. Bull. 46 (2001) 19-32.

[Esc2] J.Eschenburg, Isoparametric submanifolds and symmetric spaces, in Geometry and topology, pp.145-155, Contemp. Math., vol. 258, Amer. Math. Soc., 2000.

[EsH] J.Eschenburg, E.Heintze, Polar representations and symmetric spaces, J. Reine Angew. Math. 507 (1999) 93-106.

[Esk] A.Eskin, Quasi-isometric rigidity of nonuniform lattices in higher rank symmetric spaces, J. Amer. Math. Soc. 11 (1998), 321-361.

[EF1] A.Eskin, B.Farb, Quasi-flats in $H^{2} \times H^{2}$, in Lie groups and ergodic theory, pp. 75-103, Tata Inst. Fund. Res. Stud. Math., 14, Tata Inst. Fund. Res., 1998.

[EF2] A.Eskin, B.Farb, Quasi-flats and rigidity in higher rank symmetric spaces, J. Amer. Math. Soc. 10 (1997) 653-692.

[Fa] B.Farb, The quasi-isometry classification of lattices in semisimple Lie groups, Math. Res. Lett. 4 (1997) 705-717.

[FS] B.Farb, R.Schwartz, The large-scale geometry of Hilbert modular groups, J. Differential Geom. 44 (1996) 435-478.

[FJO] F.Farrell, L.Jones, P.Ontaneda, Hyperbolic manifolds with negatively curved exotic triangulations in dimensions greater than five, J. Differential Geom. 48 (1998) 319-322. 
[FJ] C.Favre, M.Jonsson, The valuative tree, Lecture Notes in Mathematics, vol. 1853, SpringerVerlag, 2004. xiv+234 pp.

[FKM] D.Ferus, H.Karcher, H.Munzner, Cliffordalgebren und neue isoparametrische Hyperfldchen, Math. Z. 177 (1981) 479-502.

[FRR] S. Ferry, A. Ranicki, J. Rosenberg, Novikov conjectures, index theorems and rigidity, vol. $1 \& 2$, Cambridge University Press, 1995.

[FvP] J.Fresnel, M.van der Put, Rigid analytic geometry and its applications, Progress in Mathematics, vol. 218, Birkhäuser Boston, 2004, xii+296 pp.

[FW] E.Friedlander, C.Weibel, An overview of algebraic K-theory, in Algebraic K-theory and its applications, World Sci. Publishing, 1999.

[Fu1] H.Furstenberg, A Poisson formula for semi-simple Lie groups, Ann. of Math. 77 (1963) 335-386.

[Fu2] H.Furstenberg, Boundaries of Lie groups and discrete subgroups, in Actes du Congrhs International des Mathimaticiens (Nice, 1970), Tome 2, pp. 301-306, Gauthier-Villars, 1971.

[Fu3] H.Furstenberg, Poisson boundaries and envelopes of discrete groups, Bull. Amer. Math. Soc. 73 (1967) 350-356.

[GLP] D.Gaboriau, G.Levitt, F.Paulin, Pseudogroups of isometries of $R$ and Rips' theorem on free actions on R-trees, Israel J. Math. 87 (1994) 403-428.

[GY] W.Gan, J.Yu, Schimas en groupes et immeubles des groupes exceptionnels sur un corps local. I. Le groupe $G_{2}$. [Group schemes and buildings of exceptional groups over a local field. I. The group $G_{2}$ ], Bull. Soc. Math. France 131 (2003) 307-358.

[Garl] H.Garland, p-adic curvature and the cohomology of discrete subgroups of p-adic groups, Ann. of Math. 97 (1973) 375-423.

[GaR] H.Garland, M.S.Raghunathan, A Bruhat decomposition for the loop space of a compact group: a new approach to results of Bott, Proc. Nat. Acad. Sci. U.S.A. 72 (1975) 4716-4717.

[Garr] P.Garrett, Buildings and classical groups, Chapman \& Hall, London, 1997.

[Ge] P.Gerardin, Harmonic functions on buildings of reductive split groups, in Operator algebras and group representations, Vol. I, pp. 208-221, Monogr. Stud. Math., 17, Pitman, 1984.

[GvP] P.Gerritzen, M.van der Put, Schottky groups and Mumford curves, Lecture Notes in Mathematics, vol. 817, Springer, 1980, viii+317 pp.

[GL] P.Girardin, K.Lai, Asymptotic behaviour of eigenfunctions on semi-homogeneous tree, Pacific J. Math. 196 (2000) 415-427.

[Gol] B.Goldfarb, Novikov conjectures for arithmetic groups with large actions at infinity, $K$ Theory 11 (1997) 319-372.

[GHM] M.Goresky, G.Harder, R.MacPherson, Weighted cohomology, Invent. Math. 116 (1994) 139-213. 
[Gra1] D.Grayson, Reduction theory using semistability. II, Comment. Math. Helv. 61 (1986) 661-676.

[Gra2] D.Grayson, Reduction theory using semistability, Comment. Math. Helv. 59 (1984) 600634.

[Gra3] D.Grayson, Finite generation of K-groups of a curve over a finite field (after Daniel Quillen), in Algebraic K-theory, Part I, pp. 69-90, Lecture Notes in Math., vol. 966, Springer, 1982.

[GP] M.Gromov, P.Pansu, Rigidity of lattices: an introduction, in Geometric topology: recent developments, pp. 39-137, Lecture Notes in Math., 1504, Springer, 1991.

[Gr] M.Gromov, Asymptotic invariants of infinite groups, in Geometric group theory, Vol. 2, pp. 1-295, London Math. Soc. Lecture Note Ser., vol. 182, Cambridge Univ. Press, 1993.

[GS] M.Gromov, R.Schoen, Harmonic maps into singular spaces and p-adic superrigidity for lattices in groups of rank one, Inst. Hautes Itudes Sci. Publ. Math. 76 (1992) 165-246.

[GJT] Y.Guivarch, L.Ji, J.C.Taylor, Compactifications of symmetric spaces, in Progress in Mathematics, vol. 156, Birkhäuser, Boston, 1998.

[Ha] P. de la Harpe, Topics in geometric group theory, Chicago University Press, 2000.

[HV] P. de la Harpe, A.Valette, La propriiti $(T)$ de Kazhdan pour les groupes localement compacts, Astérique No. 175, (1989), 158 pp.

[Har] M.Harris, A characterization of odd order extensions of the finite projective symplectic groups PSp(6, q), q odd, Comm. Algebra 2 (1974) 133-142.

[HZ1] M.Harris, S.Zucker, Boundary cohomology of Shimura varieties. II. Hodge theory at the boundary Invent. Math. 116 (1994) 243-308.

[HZ2] M.Harris, S.Zucker, Boundary cohomology of Shimura varieties. I. Coherent cohomology on toroidal compactifications, Ann. Sci. Icole Norm. Sup. 27 (1994) 249-344.

[Hat1] T.Hattori, Asymptotic geometry of arithmetic quotients of symmetric spaces, Math. Z. 222 (1996) 247-277.

[Hat2] T.Hattori, Collapsing of quotient spaces of $\mathrm{SO}(n) \backslash \mathrm{SL}(n, R)$ at infinity, J. Math. Soc. Japan 47 (1995) 193-225.

[HeL] E.Heintze, X.Liu, Homogeneity of infinite-dimensional isoparametric submanifolds, Ann. of Math. 149 (1999) 149-181.

[HPTT] E.Heintze, R.Palais, C.Terng, G.Thorbergsson, Hyperpolar actions and $k$-flat homogeneous spaces, J. Reine Angew. Math. 454 (1994) 163-179.

[He] D.Herscovici, Semimodular lattices and semibuildings, Journal of algebraic combinatorics 7 (1998) 39-51.

[Hij] H.Hijikata, On the structure of semi-simple algebraic groups over valuation fields. I., Japan J. Math. (N.S.) 1 (1975) 225-300. 
[Hir] J.Hirschfeld, Projective geometries over finite fields, Second edition, Oxford Mathematical Monographs, Oxford University Press, 1998. xiv+555 pp.

[HKW] K.Hulek, C.Kahn, S.Weintraub, Moduli spaces of abelian surfaces: compactification, degenerations, and theta functions, Walter de Gruyter \& Co. 1993. xii+347 pp.

[HS1] C.Hummel, V.Schroeder, Tits geometry of cocompact real-analytic Hadamard manifolds of dimension 4, Differential Geom. Appl. 11 (1999) 129-143.

[HS2] C.Hummel, V.Schroeder, Tits geometry associated with 4-dimensional closed real-analytic manifolds of nonpositive curvature, J. Differential Geom. 48 (1998) 531-555.

[Hu] J.Humphreys, Reflection groups and Coxeter groups, Cambridge University Press, 1990.

[Iv] N.Ivanov, Action of Mvbius transformations on homeomorphisms: stability and rigidity, Geom. Funct. Anal. 6 (1996) 79-119.

[Iw] N.Iwahori, Generalized Tits system (Bruhat decomposition) on p-adic semisimple groups, in Algebraic Groups and Discontinuous Subgroups, Proc. Sympos. Pure Math., vol. IX, pp. 71-83, Amer. Math. Soc., 1966.

[IM] N.Iwahori, H.Matsumoto, On some Bruhat decomposition and the structure of the Hecke rings of p-adic Chevalley groups, Inst. Hautes Itudes Sci. Publ. Math. 25 (1965) 5-48.

[Ja] T.Januszkiewicz, For Coxeter groups $z^{|g|}$ is a coefficient of a uniformly bounded representation, Fund. Math. 174 (2002) 79-86.

[JS] T.Januszkiewicz, J.Świątkowski, Hyperbolic Coxeter groups of large dimension, Comment. Math. Helv. 78 (2003) 555-583.

[J1] L.Ji, Integral Novikov conjectures for S-arithmetic groups I, preprint.

[J2] L.Ji, Asymptotic dimension and the integral K-theoretic Novikov conjecture for arithmetic groups, J. Diff. Geom, 68 (2004) 535-544.

[J3] L.Ji, Metric compactifications of locally symmetric spaces, International J. of Math. 9 (1998) 465-491.

[J4] L.Ji, Integral Novikov conjectures for S-arithmetic groups II, preprint.

[JM] L.Ji, R.MacPherson, Geometry of compactifications of locally symmetric spaces Ann. Inst. Fourier, Grenoble 52 (2002) 457-559.

[JoM] D.Johnson, J.Millson, Deformation spaces associated to compact hyperbolic manifolds, in Discrete groups in geometry and analysis, pp. 48-106, Progr. Math., 67, Birkhäuser Boston, 1987.

[Jo1] J.Jost, Nonpositive curvature: geometric and analytic aspects, Lectures in Mathematics ETH Zurich, Birkhäuser Verlag, 1997.

[Jo2] J.Jost, Generalized harmonic maps between metric spaces, in Geometric analysis and the calculus of variations, pp. 143-174, Internat. Press, 1996. 
[Jo3] J.Jost, Convex functionals and generalized harmonic maps into spaces of nonpositive curvature, Comment. Math. Helv. 70 (1995) 659-673.

[JL] J.Jost, J.Li, Finite energy and totally geodesic maps from locally symmetric spaces of finite volume, Calc. Var. Partial Differential Equations 4 (1996) 409-420.

[JY1] J.Jost, S.T.Yau, Harmonic maps and superrigidity, in Differential Geometry, Proc. Symp in Pure Math., vol. 54, Part I, 1993, pp. 245-280.

[JY2] J.Jost, S.T.Yau, Applications of quasilinear PDE to algebraic geometry and arithmetic lattices, in Algebraic geometry and related topics, pp. 169-193, Internat. Press, 1993.

[JY3] J.Jost, S.T.Yau, Harmonic mappings and Ka"hler manifolds, Math. Ann. 262 (1983) 145166.

[JZ] J.Jost, K.Zuo, Harmonic maps into Bruhat-Tits buildings and factorizations of p-adically unbounded representations of $\pi_{1}$ of algebraic varieties. I, J. Algebraic Geom. 9 (2000) 1-42.

[KLPS] W.Kantor, R.Liebler, S.Payne and E.Shult, Finite geometries, buildings, and related topics, Oxford Univ. Press, 1990.

[Kap] M.Kapovich, Hyperbolic manifolds and discrete groups, Progress in Math., vol.183, Birkhäuser, 2001.

[Kaz] D.Kazhdan, Connection of the dual space of a group with the structure of its closed subgroups, Funct. Anal. Appl. 1 (1967) 63-65.

[KKMS] G.Kempf, F.Knudsen, D.Mumford, B.Saint-Donat, Toroidal embeddings. I, Lecture Notes in Mathematics, Vol. 339. Springer-Verlag, 1973. viii+209 pp.

$[\mathrm{KM}] \quad$ J.L.Kim, A.Moy, Involutions, classical groups, and buildings, J. Algebra 242 (2001) 495515.

[Kle] F.Klein, Vergleichende Betrachtungen über neuere geometrische Forschungen (Erlangen, 1872), Math. Annalen 43 (1893) 63-100.

[Kli] M.Kline, Mathematical thought from ancient to modern times, New York, Oxford University Press, 1972.

[KL1] B.Kleiner, B.Leeb, Groups quasi-isometric to symmetric spaces, Comm. Anal. Geom. 9 (2001) 239-260.

[KL2] B.Kleiner, B.Leeb, Rigidity of quasi-isometries for symmetric spaces and Euclidean buildings, Inst. Hautes Itudes Sci. Publ. Math. 86 (1997) 115-197.

[KL3] B.Kleiner, B.Leeb, Quasi-isometries and the de Rham decomposition, Topology 37 (1998) 1193-1211.

[KlS] G.Kleineidam, J.Souto, Algebraic Convergence of Function Groups, Comm. Math. Helv. 77 (2002) 244-269.

[Klig1] B.Klingler, Sur la rigiditi de certains groupes fondamentaux, l'arithmiticiti des riseaux hyperboliques complexes, et les "faux plans projectifs", Invent. Math. 153 (2003) 105-143. 
[Klig2] B.Klingler, Un théorème de rigiditi non-mitrique pour les variitis localement symitriques hermitiennes, Comment. Math. Helv. 76 (2001) 200-217.

[Klig3] B.Klingler, Transformation de type Poisson relative aux groupes d'Iwahori, Algebraic groups and arithmetic, pp. 321-337, Tata Inst. Fund. Res., Mumbai, 2004.

[Ko] A.Koranyi, Harmonic functions on trees and buildings, Contemporary Mathematics, vol. 206, American Mathematical Society, 1997. x+181 pp.

[KS1] N.Korevaar, R.Schoen, Sobolev spaces and harmonic maps for metric space targets, Comm. Anal. Geom. 1 (1993) 561-659.

[KS2] N.Korevaar, R.Schoen, Global existence theorems for harmonic maps to non-locally compact spaces, Comm. Anal. Geom. 5 (1997) 333-387.

[Kr1] L.Kramer, Homogeneous spaces, Tits buildings, and isoparametric hypersurfaces, Mem. Amer. Math. Soc. 158 (2002), no. 752, xvi+114 pp.

[Kr2] L.Kramer, Buildings and classical groups, in Tits buildings and the model theory of groups, 59-101, London Math. Soc. Lecture Note Ser., vol. 291, Cambridge Univ. Press, 2002.

[Kr3] L.Kramer, Loop groups and twin buildings, Geom. Dedicata 92 (2002) 145-178.

[KT] L.Kramer, K.Tent, Affine $\Lambda$-buildings, ultrapowers of Lie groups and Riemannian symmetric spaces: an algebraic proof of the Margulis conjecture, preprint, math.DG/0209122.

[Laf1] L.Lafont, Rigidity results for certain 3-dimensional singular spaces and their fundamental groups, Geom. Dedicata 109 (2004) 197-219.

[Laf2] L.Lafont, Strong Jordan separation and applications to rigidity, preprint.

[Lan1] E.Landvogt, Some functorial properties of the Bruhat-Tits building, J. Reine Angew. Math. 518 (2000) 213-241.

[Lan2] E.Landvogt, A compactification of the Bruhat-Tits building, Lecture Notes in Mathematics, vol. 1619, Springer-Verlag, Berlin, 1996.

[Leb] B.Leeb, A characterization of irreducible symmetric spaces and Euclidean buildings of higher rank by their asymptotic geometry, Bonner Mathematische Schriften, vol. 326, 2000, ii +42 pp.

[Le1] E.Leuzinger, Tits geometry, arithmetic groups, and the proof of a conjecture of Siegel, J. Lie Theory 14 (2004) 317-338.

[Le2] E.Leuzinger, Geodesic rays in locally symmetric spaces, Differential Geom. Appl. 6 (1996) $55-65$.

[Li1] W.Li, Ramanujan hypergraphs, Geom. Funct. Anal. 14 (2004) 380-399.

[Li2] W.Li, Number Theory with Applications, World Scientific, Singapore, 1996.

[Li3] W.Li, A survey of Ramanujan graphs, in Arithmetic, Geometry and Coding Theory, Proceedings of International Conference held at Luminy, France, June 28 - July 2, 1993, de Gruyter, pp. 127-143, 1996. 
[Li4] W.Li, Eigenvalues of Ramanujan graphs, In Emerging Applications of Number Theory (Minneapolis, MN, 1996), pp. 387-403, IMA Vol. Math. Appl. 109, Springer, 1999.

[Lim] S.Lim, Minimal volume entropy on graphs, preprint.

[Lu] A.Lubotzky, Lattices in rank one Lie groups over local fields, Geom. Funct. Anal. 1 (1991) 406-431.

[LPS] A.Lubotzky, R.Phillips, P.Sarnak, Ramanujan graphs, Combinatorica 8 (1988) 261-277.

[LSV1] A.Lubotzky, B.Samuels, U.Vishne, Ramanujan complexes of type $\tilde{A}_{n}$, to appear in Israel Journal.

[LSV2] A.Lubotzky, B.Samuels, U.Vishne, Explicit constructions of Ramanujan complexes of type $\tilde{A}_{n}$, preprint, 2004.

[Ly] A.Lytchak, Rigidity of spherical buildings and joins, to appear in GAFA.

[MS] F.MacWilliams, N.Sloane, The theory of error-correcting codes: I \& II, North-Holland Mathematical Library, Vol. 16, North-Holland Publishing Co., 1977.

[Man] Y.Manin, Three-dimensional hyperbolic geometry as $\infty$-adic Arakelov geometry, Invent. Math. 104 (1991) 223-243.

[Mar] G.Margulis, Discrete subgroups of semisimple Lie groups, in Ergebnisse der Mathematik und ihrer Grenzgebiete, Springer-Verlag, 1991.

[Mat1] Y.Matsushima, On the first Betti number of compact quotient spaces of higher-dimensional symmetric spaces, Ann. of Math. 75 (1962) 312-330.

[Mat2] Y.Matsushima, On Betti numbers of compact, locally symmetric Riemannian manifolds Osaka Math. J. 14 (1962) 1-20.

[McS] P.McMullen, E.Schulte, Abstract regular polytopes, Encyclopedia of Mathematics and its Applications, vol. 92, Cambridge University Press, 2002. xiv+551 pp.

[Mok1] N.Mok, Aspects of Kdhler geometry on arithmetic varieties, in Several complex variables and complex geometry, Proc. Sympos. Pure Math., 52, Part 2, pp. 335-396, Amer. Math. Soc., 1991.

[Mok2] N.Mok, The holomorphic or antiholomorphic character of harmonic maps into irreducible compact quotients of polydiscs, Math. Ann. 272 (1985) 197-216.

[MT] R.Moody, K.Teo, Tits' systems with crystallographic Weyl groups, J. Algebra 21 (1972) 178-190.

[Mor1] J.Morgan, $\Lambda$-trees and their applications, Bull. Amer. Math. Soc. 26 (1992) 87-112.

[Mor2] J.Morgan, Trees and hyperbolic geometry, in Proceedings of the International Congress of Mathematicians, (Berkeley, Calif., 1986) pp. 590-597, Amer. Math. Soc., 1987.

[Mor3] J.Morgan, On Thurston's uniformization theorem for three-dimensional manifolds, in The Smith conjecture, pp. 37-125, Pure Appl. Math., vol. 112, Academic Press, 1984. 
[Mor4] J.Morgan, Group actions on trees and the compactification of the space of classes of $\mathrm{SO}(n, 1)$-representations, Topology 25 (1986) 1-33.

[MS1] J.Morgan, P.Shalen, Valuations, trees and degenerations of hyperbolic structures I, Annal of Math. 120 (1984) 401-476.

[MS2] J.Morgan, P.Shalen, Degenerations of hyperbolic structures. III. Actions of 3-manifold groups on trees and Thurston's compactness theorem, Ann. of Math. 127 (1988) 457-519.

[MS3] J.Morgan, P.Shalen, Degeneration of hyperbolic structures III: Actions of 3-manifold groups on trees and Thurston's compactification theorem, Annal of Math. 127 (1988) 457519.

[Mors] M. Morgenstern, Existence and explicit constructions of $q+1$ regular Ramanujan graphs for every prime power q, J. Comb. Theory, series B 62 (1994) 44-62.

[Mos] G.Mostow, Strong rigidity of locally symmetric spaces, Princeton University Press, 1973.

[Moy] A.Moy, Displacement functions on the Bruhat-Tits building, in The mathematical legacy of Harish-Chandra, pp. 483-499, Proc. Sympos. Pure Math., 68, Amer. Math. Soc., 2000.

[MP1] A.Moy, G.Prasad, Unrefined minimal K-types for p-adic groups, Invent. Math. 116 (1994) 393-408.

[MP2] A.Moy, G.Prasad, Jacquet functors and unrefined minimal K-types, Comment. Math. Helv. 71 (1996) 98-121.

[Mu1] D.Mumford, A new approach to compactifying locally symmetric varieties, in Discrete subgroups of Lie groups and applications to moduli, pp. 211-224, Oxford Univ. Press, 1975 .

[Mu2] D.Mumford, An analytic construction of degenerating curves over complete local rings, Compositio Math. 24 (1972) 129-174.

[Mu3] D.Mumford, geometric invariant theory, Springer-Verlag, 1965.

[Mus] G.Mustafin, Non-Archimedean uniformization, Mat. Sb. (N.S.) 105 (1978) 207-237.

[ne] Y.Neretin, The beta function of the Bruhat-Tits building and the deformation of the space $l^{2}$ on the set of p-adic lattices, Sb. Math. 194 (2003) 1775-1805.

[Ni1] V.Nikulin, Reflection groups in Lobachevskiuspaces and an identity for the denominator of Lorentzian Kac-Moody algebras, Izv. Math. 60 (1996) 305-334.

[Ni2] V.Nikulin, Discrete reflection groups in Lobachevsky spaces and algebraic surfaces, in Proc. of the International Congress of Mathematicians, pp. 654-671, Amer. Math. Soc., 1987.

[MSY] N.Mok, Y.T.Siu, S.Yeung, Geometric superrigidity, Invent. Math. 113 (1993) 57-83.

[Ol] C.Olmos, Isoparametric submanifolds and their homogeneous structures, J. Differential Geom. 38 (1993) 225-234.

[On] P.Ontaneda, Hyperbolic manifolds with negatively curved exotic triangulations in dimension six, J. Differential Geom. 40 (1994) 7-22. 
[Os] T.Oshima, A realization of Riemannian symmetric spaces, J. Math. Soc. Japan, 30 (1978), 117-132.

[Ot1] J.Otal, Thurston's hyperbolization of Haken manifolds, in Surveys in differential geometry, Vol. III, 77-194, International Press, 1998.

[Ot2] J.Otal, Le théorème d'hyperbolisation pour les variétés fibrées de dimension 3," Astérisque, vol. 235, 1996.

[PT] R.Palais, C.Terng, A general theory of canonical forms, Trans. Amer. Math. Soc. 300 (1987) 771-789.

[Pan] P.Pansu, Mitriques de Carnot-Carathiodory et quasiisomitries des espaces symitriques de rang un, Ann. of Math. 129 (1989) 1-60.

[Pau1] F. Paulin, Actions de groupes sur les arbres, Siminaire Bourbaki, Vol. 1995/96, Astérique No. 241 (1997) 97-137.

[Pau2] F. Paulin, Topologie de Gromov équivariante, structures hyperboliques et arbres réels, Invent. Math. 94 (1988) 53-80.

[Pr1] G.Prasad, Volumes of S-arithmetic quotients of semi-simple groups. With an appendix by Moshe Jarden and the author, Inst. Hautes Itudes Sci. Publ. Math. 69 (1989) 91-117.

[Pr2] G.Prasad, Lattices in semisimple groups over local fields, in Studies in algebra and number theory, pp. 285-356, Adv. in Math. Suppl. Stud., 6, Academic Press, 1979.

[Pr3] G.Prasad, Strong rigidity of Q-rank 1 lattices, Invent. Math. 21 (1973) 255-286.

[PrR1] G.Prasad, M.Raghunathan, Topological central extensions of semisimple groups over local fields, Ann. of Math. 119 (1984) 143-201.

[PrR2] G.Prasad, M.Raghunathan, Topological central extensions of semisimple groups over local fields. II, Ann. of Math. 119 (1984) 203-268.

[PYe] G.Prasad, S.Yeung, Fake projective planes, preprint.

[PYu] G.Prasad, J.K.Yu, On finite group actions on reductive groups and buildings, Invent. Math. 147 (2002) 545-560.

[Qu1] D.Quillen, Finite generation of the groups $K_{i}$ of rings of algebraic integers, in Algebraic K-theory, I: Higher K-theories, 179-198, Lecture Notes in Math., Vol. 341, Springer, 1973.

[Qu2] D.Quillen, On the cohomology and K-theory of the general linear groups over a finite field, Ann. of Math. 96 (1972) 552-586.

[Ra1] M.Raghunathan, Vanishing theorems for cohomology groups associated to discrete subgroups of semisimple Lie groups, Osaka J. Math. 3 (1966) 243-256. Corrections, Osaka J. Math. 16 (1979) 295-299.

[Ra2] M.Raghunathan, On the first cohomology of discrete subgroups of semisimple Lie groups, Amer. J. Math. 87 (1965) 103-139. 
[Ra3] M.Raghunathan, Principal bundles admitting a rational section, Invent. Math. 116 (1994) 409-423.

[RaZ] M.Rapoport, T.Zink, Period spaces for p-divisible groups, Annals of Mathematics Studies, vol. 141, Princeton University Press, 1996, xxii+324 pp.

[Re1] B.Remy, Kac-Moody groups: split and relative theories. Lattices, in Groups: topological, combinatorial and arithmetic aspects, pp. 487-541, London Math. Soc. Lecture Note Ser., 311, Cambridge Univ. Press, Cambridge, 2004.

[Re2] B.Remy, Immeubles de Kac-Moody hyperboliques, groupes non isomorphes de mjme immeuble, Geom. Dedicata 90 (2002) 29-44.

[Re3] B.Remy, Groupes de Kac-Moody diployis et presque diployis, Astérique No. 277 (2002), viii +348 pp.

[Re4] B.Remy, Construction de riseaux en thiorie de Kac-Moody, C. R. Acad. Sci. Paris Sir. I Math. 329 (1999) 475-478.

[RS] J.Rohlfs, T.Springer, Applications of buildings, in Handbook of incidence geometry, 10851114, North-Holland, 1995.

[Ro1] M.Ronan, Buildings: main ideas and applications. I. Main ideas, Bull. London Math. Soc. 24 (1992) 1-51.

[Ro2] M.Ronan, Buildings: main ideas and applications. II. Arithmetic groups, buildings and symmetric spaces, Bull. London Math. Soc. 24 (1992) 97-126.

[Ro3] M.Ronan, Lectures on buildings. Perspectives in Mathematics, vol. 7, Academic Press, 1989, xiv+201 pp.

[Ros] A. Rosati, Buildings and the geometry of diagrams, Lecture Notes in Mathematics, vol. 1181, Springer-Verlag, 1986. viii+269 pp.

[Rose] D.Rosenthal, Splitting with continuous control in algebraic K-theory, K-Theory 32 (2004) 139-166.

[RT] M.Ronan, J.Tits, Building buildings, Math. Ann. 278 (1987) 291-306.

[Ru] W.Ruan, On the convergence and collapsing of Kdhler metrics, J. Differential Geom. 52 (1999) 1-40.

[Sa] J.Sampson, Applications of harmonic maps to Kähler geometry, in Complex differential geometry and nonlinear differential equations, pp. 125-134, Contemp. Math., vol. 49, Amer. Math. Soc., 1986.

[Sar] A.Sarveniazi, Ramanujan $\left(n_{1}, n_{2}, \ldots, n_{d-1}\right)$-regular hypergraphs based on Bruhat-Tits buildings of type $\tilde{A}_{d-1}$, Ph. D. Thesis, U. Göttingen, 2004.

[Sca] R.Scharlau, Buildings, in Handbook of incidence geometry, pp. 477-645, North-Holland, 1995.

[Scr1] R.Schwartz, Quasi-isometric rigidity and Diophantine approximation, Acta Math. 177 (1996) 75-112. 
[Scr2] R.Schwartz, The large-scale geometry of Hilbert modular groups, J. Differential Geom. 44 (1996) 435-478.

[SS1] P.Schneider, U.Stuhler, Representation theory and sheaves on the Bruhat-Tits building, Inst. Hautes Itudes Sci. Publ. Math. 85 (1997) 97-191.

[SS2] P.Schneider, U.Stuhler, The cohomology of p-adic symmetric spaces, Invent. Math. 105 (1991) 47-122.

[ST] P.Schneider, J.Teitelbaum, p-adic boundary values, in Cohomologies p-adiques et applications arithmitiques, Astérique No. 278 (2002) 51-125.

[Sce] R.Schoen, The role of harmonic mappings in rigidity and deformation problems, in Complex geometry (Osaka, 1990), pp. 179-200, Lecture Notes in Pure and Appl. Math., 143, Dekker, 1993.

[SY] R.Schoen, S.T.Yau, On univalent harmonic maps between surfaces, Invent. Math. 44 (1978) 265-278.

[Se1] J.P.Serre, Trees, Springer-Verlag, 1980.

[Se2] J.P.Serre, Cohomologie des groupes discrets, in Prospects in mathematics, pp. 77-169. Ann. of Math. Studies, No. 70, Princeton Univ. Press, 1971.

[Shn] P.Shalen, Dendrology and its applications, in Group theory from a geometrical viewpoint, pp. 543-616, World Sci. Publishing, 1991.

[Shm1] Y.Shalom, Rigidity of commensurators and irreducible lattices, Invent. Math. 141 (2000) $1-54$.

[Shm2] Y.Shalom, Rigidity, unitary representations of semisimple groups, and fundamental groups of manifolds with rank one transformation group, Ann. of Math. 152 (2000) 113-182.

[Sim] C.Simpson, The ubiquity of variations of Hodge structure, in Complex geometry and Lie theory, pp. 329-348, Proc. Sympos. Pure Math., vol. 53, Amer. Math. Soc., 1991.

[Siu1] Y.Siu, Strong rigidity of compact quotients of exceptional bounded symmetric domains, Duke Math. J. 48 (1981) 857-871.

[Siu2] Y.Siu, The complex-analyticity of harmonic maps and the strong rigidity of compact Kdhler manifolds, Ann. of Math. 112 (1980) 73-111.

[Sk] R.Skora, Splittings of surfaces, J. Amer. Math. Soc. 9 (1996) 605-616.

[So] L.Solomon, The Steinberg character of a finite group with BN-pair, in Theory of Finite Groups (Symposium, Harvard Univ.), pp. 213-221, Benjamin, New York, 1969.

[Sp1] R.Spatzier, An invitation to rigidity theory, in Modern dynamical systems and applications, pp. 211-231, Cambridge Univ. Press, Cambridge, 2004

[Sp2] R.Spatzier, Harmonic analysis in rigidity theory, in Ergodic theory and its connections with harmonic analysis, pp. 153-205, London Math. Soc. Lecture Note Ser., vol. 205, Cambridge Univ. Press, 1995. 
[Su1] M.Suzuki, Characterizations of some finite simple groups, Actes du Congrhs International des Mathimaticiens (Nice, 1970), Tome 1, pp. 371-373. Gauthier-Villars, 1971.

[Su2] M.Suzuki, Characterizations of linear groups, Bull. Amer. Math. Soc. 75 (1969) 1043-1091.

[Su3] M.Suzuki, Finite groups in which the centralizer of any element of order 2 is 2-closed, Ann. of Math. 82 (1965) 191-212.

[Te] J.Teitelbaum, The geometry of p-adic symmetric spaces, Notices Amer. Math. Soc. 42 (1995) 1120-1126.

[Ter1] C.Terng, Recent progress in submanifold geometry, in Differential geometry, pp. 439-484, Proc. Sympos. Pure Math., 54, Part 1, Amer. Math. Soc. 1993.

[Ter2] C.Terng, Isoparametric submanifolds and their Coxeter groups, J. Differential Geom. 21 (1985) 79-107.

[Tha1] J.Thas, Generalized polygons, in Handbook of incidence geometry, pp. 383-431, NorthHolland, 1995.

[Tha2] J.Thas, Projective geometry over a finite field, in Handbook of incidence geometry, pp. 295-347, North-Holland, 1995.

[Tho1] G.Thorbergsson, A survey on isoparametric hypersurfaces and their generalizations, in Handbook of differential geometry, Vol. I, pp. 963-995, North-Holland, 2000.

[Tho2] G.Thorbergsson, Isoparametric foliations and their buildings, Ann. of Math. 133 (1991) 429-446.

[Tho3] G.Thorbergsson, Clifford algebras and polar planes, Duke Math. J. 67 (1992) 627-632.

[Thu1] W.Thurston, Hyperbolic structures on 3-manifolds. I. Deformation of acylindrical manifolds, Ann. of Math. 124 (1986) 203-246.

[Thu2] W.Thurston, Hyperbolic geometry and 3-manifolds, in Low-dimensional topology, pp. 9-25, London Math. Soc. Lecture Note Ser., 48, Cambridge Univ. Press, 1982.

[Thu3] W.Thurston, Three-dimensional manifolds, Kleinian groups and hyperbolic geometry, Bull. Amer. Math. Soc. 6 (1982) 357-381.

[Ti1] J.Tits, On buildings and their applications, in Proceedings of the International Congress of Mathematicians (Vancouver, B. C., 1974), Vol. 1, pp. 209-220, Canad. Math. Congress, 1975 .

[Ti2] J.Tits, Buildings of spherical type and finite BN-pairs, Lecture Notes in Mathematics, Vol. 386, Springer-Verlag, 1974, 299 pp.

[Ti3] J.Tits, Reductive groups over local fields, in Automorphic forms, representations and Lfunctions, Part 1, pp. 29-69, Proc. Sympos. Pure Math., XXXIII, Amer. Math. Soc., 1979.

[Ti4] J.Tits, Les groupes de Lie exceptionnels et leur interpritation giomitrique, Bull. Soc. Math. Belg. 8 (1956) 48-81. 
[Ti5] J.Tits, Sur certaines classes d'espaces homogènes de groupes de Lie, Mém. Acad. Roy. Gelg. 29 (3), 1955.

[Ti6] J.Tits, Groupes semi-simples complexes et gémétrie projective, Séminaire Bourbaki, exposé, No. 112, 1955.

[Ti7] J.Tits, Immeubles de type affine, in Buildings and the geometry of diagrams, pp. 159-190, Lecture Notes in Math., vol. 1181, Springer, 1986.

[TW] J.Tits, R.Weiss, Moufang polygons, Springer-Verlag, 2002, x+535 pp.

[vP] M.van der Put, Discrete groups, Mumford curves and theta functions, Ann. Fac. Sci. Toulouse Math. 1 (1992) 399-438.

[vPV] M.van der Put, H.Voskuil, Symmetric spaces associated to split algebraic groups over a local field, J. Reine Angew. Math. 433 (1992) 69-100.

[Vi] E.Vinberg, Discrete reflection groups in Lobachevsky spaces, in Proc. of the International Congress of Mathematicians, pp. 593-601, Warsaw, 1984.

[VS] E.Vinberg, O.Shvartsman, Discrete groups of motions of spaces of constant curvature, in Geometry II, pp. 139-248, Encyclopaedia Math. Sci., 29, Springer, 1993.

[Wa] S.Wang, On isolated points in the dual spaces of locally compact groups, Math. Ann. 218 (1975) 19-34

[Was] M.Wachs, Quotients of Coxeter complexes and buildings with linear diagram, European J. Combin. 7 (1986) 75-92.

[Wei1] A.Weil, Remarks on the cohomology of groups, Ann. of Math. 80 (1964) 149-157.

[Wei2] A.Weil, On discrete subgroups of Lie groups. II, Ann. of Math. 75 (1962) 578-602.

[Wei3] A.Weil, On discrete subgroups of Lie groups, Ann. of Math. 72 (1960) 369-384.

[Wer1] A.Werner, Compactification of the Bruhat-Tits building of PGL by seminorms, Math. Z. 248 (2004) 511-526.

[Wer2] A.Werner, Arakelov intersection indices of linear cycles and the geometry of buildings and symmetric spaces, Duke Math. J. 111 (2002) 319-355.

[Wer3] A.Werner, Compactification of the Bruhat-Tits building of PGL by lattices of smaller rank, Doc. Math. 6 (2001) 315-341.

[Woe] W.Woess, Random walks on infinite graphs and groups, in Cambridge Tracts in Mathematics, vol. 138, Cambridge University Press, 2000.

[Wol1] M.Wolf, Harmonic maps from surfaces to R-trees, Math. Z. 218 (1995) 577-593.

[Wol2] M.Wolf, Infinite energy harmonic maps and degeneration of hyperbolic surfaces in moduli space, J. Differential Geom. 33 (1991) 487-539.

[Wol3] M.Wolf, The Teichmüller theory of harmonic maps, J. Differential Geom. 29 (1989) 449479. 
[Yag] I.Yaglom, Felix Klein and Sophus Lie, Birkhäuser, 1988.

[Yau1] S.T.Yau, The current state and prospects of geometry and nonlinear differential equations, in Mathematical research today and tomorrow, Lecture Notes in Mathematics, vol. 1525, pp. 29-39, 1992.

[Yau2] S.T.Yau, Problem section, in Seminar on Differential Geometry, pp. 669-706, Ann. of Math. Stud., 102, Princeton Univ. Press, 1982.

[Yau3] S.T.Yau, Harmonic maps between Riemannian manifolds, in Partial differential equations and geometry, pp. 307-311, Lecture Notes in Pure and Appl. Math., 48, Dekker, 1979.

[Yau4] S.T.Yau, Calabi's conjecture and some new results in algebraic geometry, Proc. Nat. Acad. Sci. U.S.A. 74 (1977) 1798-1799.

[Ye1] S.Yeung, Integrality and Arithmeticity of Co-compact Lattice Corresponding to Certain Complex Two-ball Quotients of Picard Number One, Asian Jour. Math. 8 (2004) 107-130.

[Ye2] S.Yeung, Virtual first Betti number and integrality of compact complex two-ball quotients, Int. Math. Res. Not. 38 (2004) 1967-1988.

[Ye3] S.Yeung, On vanishing theorems and rigidity of locally symmetric manifolds, Geom. Funct. Anal. 11 (2001) 175-198.

[Yu1] J.Yu, An introduction to explicit Bruhat-Tits theory, Lecture Notes, Banff, 2001.

[Yu2] J.Yu, Bruhat-Tits theory and buildings, Lecture Notes at University of Ottawa, 2004.

[Zi] R.Zimmer, Ergodic theory and semisimple groups, Birkhäuser, 1984. 